\title{
Modeling and Integration of Demand Response and Demand Side Resources for Smart Grid Application in Distribution Systems
}

\author{
Naveen Venkatesan \\ West Virginia University
}

Follow this and additional works at: https://researchrepository.wvu.edu/etd

\section{Recommended Citation}

Venkatesan, Naveen, "Modeling and Integration of Demand Response and Demand Side Resources for Smart Grid Application in Distribution Systems" (2011). Graduate Theses, Dissertations, and Problem Reports. 606.

https://researchrepository.wvu.edu/etd/606

This Thesis is protected by copyright and/or related rights. It has been brought to you by the The Research Repository @ WVU with permission from the rights-holder(s). You are free to use this Thesis in any way that is permitted by the copyright and related rights legislation that applies to your use. For other uses you must obtain permission from the rights-holder(s) directly, unless additional rights are indicated by a Creative Commons license in the record and/ or on the work itself. This Thesis has been accepted for inclusion in WVU Graduate Theses, Dissertations, and Problem Reports collection by an authorized administrator of The Research Repository @ WVU. For more information, please contact researchrepository@mail.wvu.edu. 


\title{
Modeling and Integration of Demand Response and Demand Side Resources for Smart Grid Application in Distribution Systems
}

\author{
Naveen Venkatesan \\ Thesis submitted to the \\ College of Engineering and Mineral Resources at \\ West Virginia University \\ in partial fulfillment of the requirements for the \\ degree of \\ Master of Science \\ in \\ Electrical Engineering \\ Prof. Jignesh Solanki, Ph.D., Chair \\ Prof. Sarika Khushalani Solanki, Ph.D. \\ Prof. Ali Feliachi, Ph.D.
}

Lane Department of Computer Science and Electrical Engineering

Morgantown, West Virginia

2011

Keywords: Demand Response, Demand Side Resources, Smart Grid, Microgrid, OpenDSS

Copyright 2011 Naveen Venkatesan 


\title{
ABSTRACT \\ Modeling and Integration of Demand Response and Demand Side Resources for Smart Grid Application in Distribution Systems
}

\author{
Naveen Venkatesan
}

Todayes electric grid is undergoing drastic changes to evolve into a smart grid. Deregulation of the integrated and monopolistic power system into genco, transco and disco has led to tremendous competition among these players. These entities are in the process of developing innovative smart grid strategies that can improve their reliability and profit. In this thesis work, some of the smart grid initiatives by discos have been explored.

This thesis work is driven by two major objectives. The primary objective is to explore Demand Response (DR), develop its comprehensive model and to analyze various effects and implications of DR on distribution networks. The second major objective of the thesis is to integrate the developed demand response model into a microgrid market optimization. A microgrid network is a real world demonstration of smart grid that integrates and coordinates various demand side resources into its operation. For this reason, a microgrid has been chosen in this work so that it offers a broader scope where in addition to DR models, Battery Energy Storage System (BESS) and Distributed Energy Resources (DER) or Distributed Generation (DG) can also be modeled and integrated.

This thesis develops a model for DR by utilizing consumer behavior modeling considering different scenarios and levels of consumer rationality. Consumer behavior modeling has been done by developing extensive demand-price elasticity matrices for different types of consumers. These Price Elasticity Matrices (PEMs) are utilized to calculate the level of demand response for a given consumer. DR thus obtained is applied to a real world distribution network considering a day-ahead real time pricing scenario to study the effects of demand reduction and redistribution on system voltage and losses. Results show considerable boost in system voltage that paves way for further demand curtailment through demand side management techniques like Volt/Var Control (VVC).

Following this, the thesis develops a market optimization model for an islanded microgrid that includes Smart Grid elements namely DR, DERs and BESS. Comprehensive models for DR and BESS have been developed and integrated into the optimization program. Demand Side Bidding (DSB) by DR Aggregators is introduced into the proposed double sided microgrid energy market by utilizing the DR models developed. The optimization program uses Linear Programming (LP) technique to determine the dispatch schedule of DERs, BESS and the level of DR to minimize the operating cost of the microgrid market. A time series simulation of a large microgrid test system is performed to show the feasibility of the proposed market optimization. 


\section{ACKNOWLEDGEMENTS}

First of all, I would like to thank my esteemed mentor and advisor, Dr. Jignesh Solanki who has given me this great opportunity to do quality research in my field of interest - Power Systems. Without his constant support and motivation I could not have completed my research work and thesis dissertation. Next, I would like to thank my second committee member Dr. Sarika Khushalani Solanki. It was from her that I learned the difference between mere hard work and hard work with focus and dedication. Both Dr. Jignesh Solanki and Dr. Sarika Khushalani Solanki have been with me, guiding me throughout my two years of research. I would also like to thank my other committee member Dr. Ali Feliachi whose feedback and reviews helped me improve the quality of this thesis.

I would like to express my thanks to my friend and colleague Vaidyanath Ramachandran for all his help in each and every phase of my thesis. His adeptness with simulation tools and software has been of great help to me whenever I was stranded at some stage of my research work. A special thanks to all my friends in the APERC lab with whom I have shared research ideas and office space as well.

I would like to thank my parents for their moral support throughout my graduate studies. Their love and affection have helped me overcome the toughest of challenges during these two hard years.

I would also like to extend my sincere thanks to Mr. Roger Dugan from EPRI for providing an excellent training session in OpenDSS, the tool that I used extensively in my thesis.

This research work was partially supported by the grant provided from the Program to Stimulate Competitive Research (PSCoR) from West Virginia University Research Corporation. 


\section{CONTENTS}

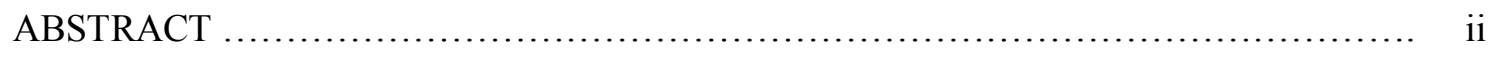

ACKNOWLEDGEMENTS..................................................... iii

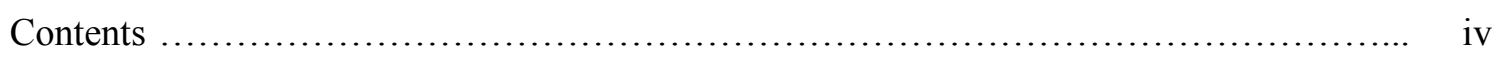

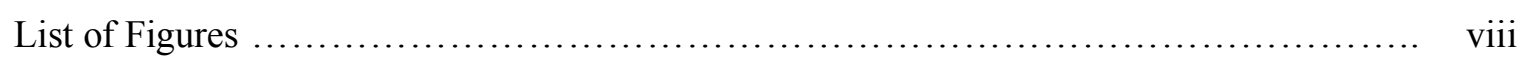

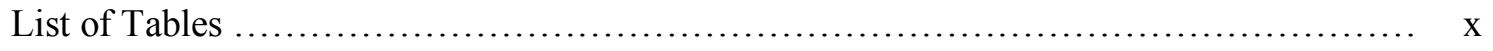

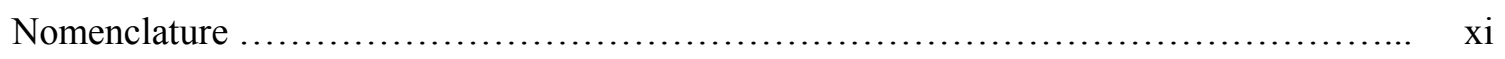

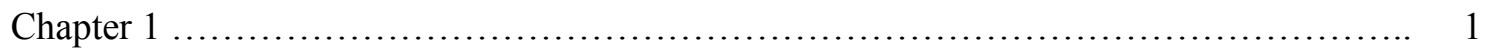

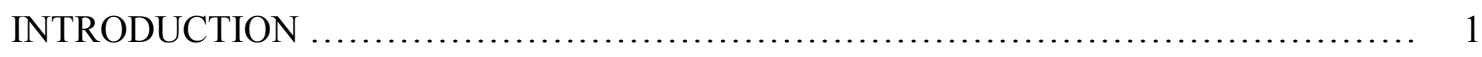

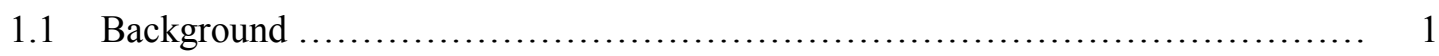

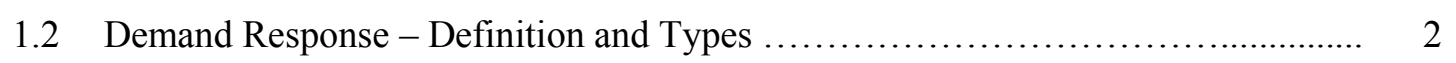

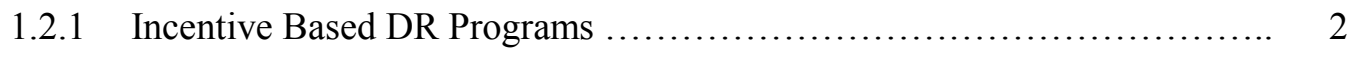

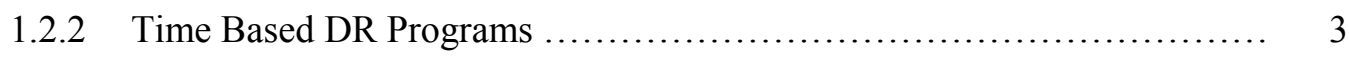

1.2.3 Day Ahead Real Time Pricing Programs .......................... 4

1.3 Demand Response - Benefits and Challenges ............................. 4

1.3.1 Benefits .................................................. 4

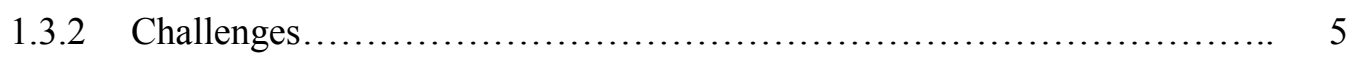

1.4 Demand Side Resources.............................................. 5

1.4.1 Distributed Generators........................................ 6

1.4.2 Battery Energy Storage Systems (BESS)......................... 6

1.5 Microgrids......................................................... 7

1.5.1 Microgrid Control Operation.................................... 7 
1.5.2 Microgrid Controller Architecture................................. 8

1.5.3 Energy Management in a Microgrid.................................... 9

1.5.4 Microgrid Modeling and Simulation................................ 9

1.6 Demand Side Bidding and Load Serving Entities........................... 10

$1.7 \quad$ Problem Statement.......................................................... 10

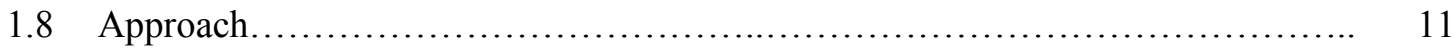

1.8.1 DR Modeling.................................................. 11

1.8.2 Microgrid Market Optimization.................................. 11

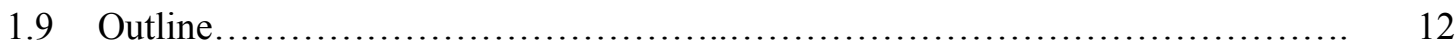

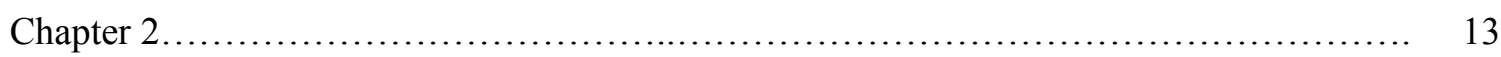

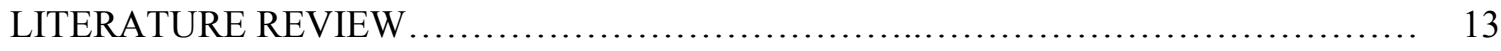

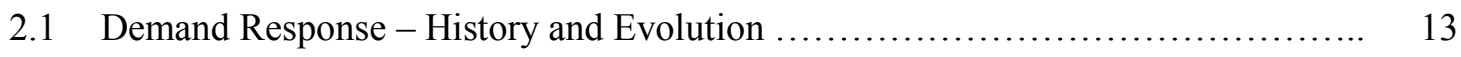

2.2 Demand Response and Consumer Behavior Modeling ....................... 13

2.3 Load Forecast with Demand Response.................................. 14

2.4 Demand Response and Smart Grid...................................... 15

2.5 Microgrids and Distributed Energy Resources $\ldots \ldots \ldots \ldots \ldots \ldots \ldots \ldots \ldots \ldots \ldots \ldots \ldots \ldots \ldots \ldots \ldots \ldots \ldots$

2.5.1 Practical Demonstrations of Microgrid.............................. 17

2.5.1.1 The EU Microgrid Research Project...................... 17

2.5.1.2 CERTS Microgrid ................................. 17

2.5.1.3 Microgrid Research Activities in Japan..................... 18

2.5.2 Microgrid Energy Management .................................... 18

2.6 Battery Energy Storage System........................................ 19

2.6.1 Early Works on BESS........................................ 19 
2.6.2 BESS in Microgrid............................................ 20

2.6.3 BESS - Optimal Schedule with Variable Tariff......................... 20

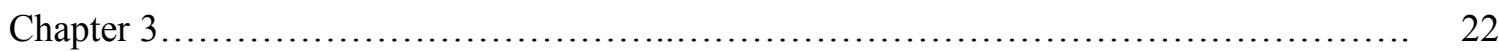

MATHEMATICAL MODEL AND FORMULATION.................................. 22

3.1 Modeling of Demand Response....................................... 22

3.1.1 Price Elasticity Matrix - Fundamentals............................ 22

3.1.2 Consumer Rationality Assumptions................................ 23

3.1.3 Long Range or Optimizing Consumers........................... 25

3.1.4 Short Range Consumers......................................... 26

3.1.5 Real World Postponing Consumer................................ 26

3.1.6 Real World Advancing Consumer................................. 26

3.1.7 Real World- Mixed Consumers................................... 27

3.2 Microgrid Market Optimization........................................... 33

3.2.1 DR Aggregator............................................. 33

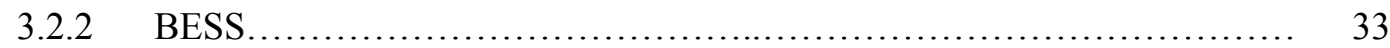

3.2.3 DER, DR and BESS Dispatch................................ 34

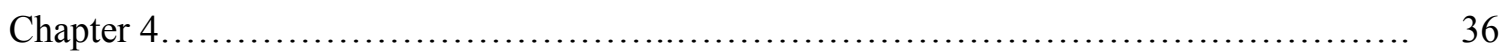

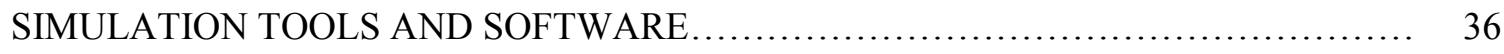

4.1 Open Distribution System Simulator (OpenDSS) ......................... 37

4.1.1 Wide Range of Solution Modes.................................. 38

4.1.2 COM Interface............................................... 39

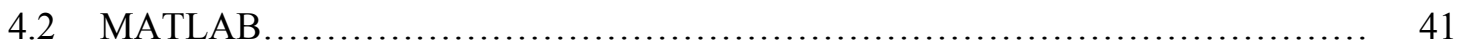


4.2.1 MATLAB Optimization Toolbox................................. 41

$4.3 \quad$ IBM ILOG CPLEX Optimizer......................................... 42

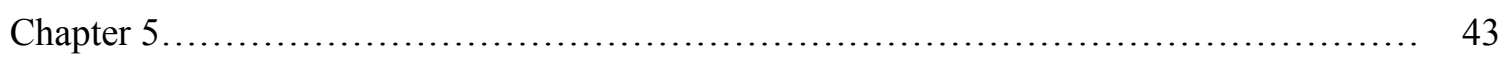

SIMULATION AND RESULTS ................................................ 43

$5.1 \quad$ IEEE 123 Node Radial Feeder......................................... 43

5.2 Extraction of DR Patterns from PEMs for IEEE 123 Node Feeder................. 45

5.3 Voltage Analysis of IEEE 123 Node Test Feeder........................... 47

$5.4 \quad$ IEEE 8500 Node Test Feeder............................................ 48

5.4.1 Voltage Analysis Results ....................................... 50

5.4.2 Loss Analysis Results...................................... 53

$5.5 \quad$ Microgrid Market Optimization........................................... 55

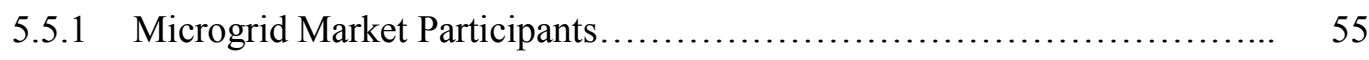

5.5.2 Market Optimization Results................................... 56

5.5.3 Microgrid Simulation............................................ 61

5.6 Microgrid Optimization and Simulation for 8500 Node Test Feeder............... 62

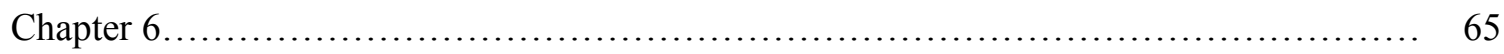

CONCLUSION AND FUTURE WORK ........................................ 66

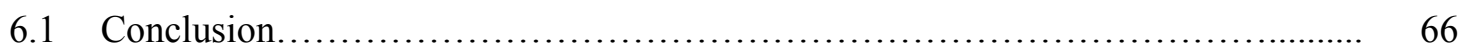

6.1.1 Demand Response Model..................................... 66

6.1.2 Microgrid Market Optimization.................................. 67

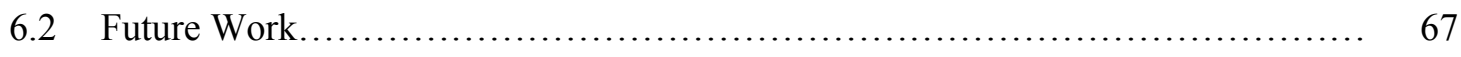

References.......................................................... 69 


\section{LIST OF FIGURES}

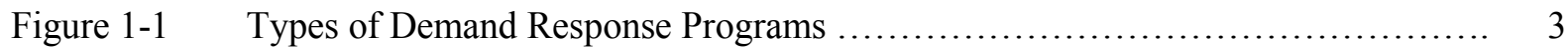

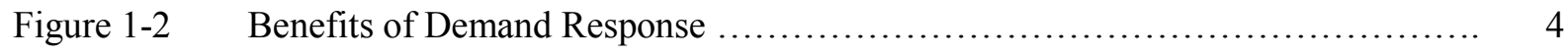

Figure 1-3 Types of Energy Storage Systems with Application........................ 7

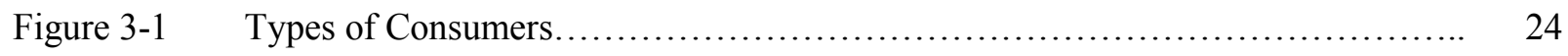

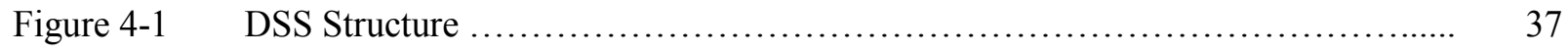

Figure 4-2 OpenDSS Standalone EXE User Interface .............................. 38

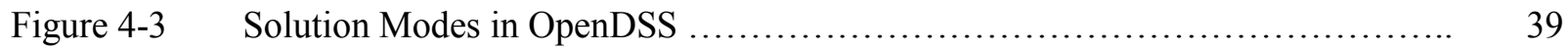

Figure 4-4 MATLAB-OpenDSS COM Interface ................................ 40

Figure 4-5 Snapshot showing MATLAB commands driving OpenDSS Engine using "text" object of COM Interface ............................................. 41

Figure 4-6 A snapshot of a simple LP formulation along with its execution in CPLEX....... 42

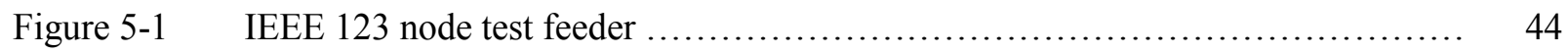

Figure 5-2 Daily demand curve of zone 3 loads for a LR consumer $\ldots \ldots \ldots \ldots \ldots \ldots \ldots \ldots \ldots \ldots$

Figure 5-3 Daily demand curve of zone 3 loads for an SR consumer $\ldots \ldots \ldots \ldots \ldots \ldots \ldots \ldots \ldots$

Figure 5-4 Daily demand curve of zone 3 loads for a RW Mixed consumer........................... 46

Figure 5-5 Comparison of Voltage profile of system nodes with and without DR................. 48

Figure 5-6 IEEE 8500 node test feeder showing section with DR loads................... 49

Figure 5-7 IEEE 8500 node test feeder - Split Phase Transformer and Residential Load

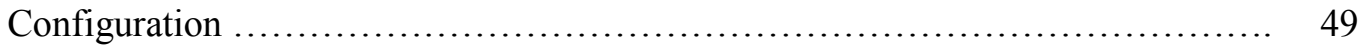

Figure 5-8 Voltage profile at node SX3047289A-Phase 1 during peak pricing hours............ 51

Figure 5-9 Voltage profile at node SX3047289A-Phase 1 during off peak hours............. 51

Figure 5-10 Voltage profile at node SX3029498C-Phase 1 during peak pricing hours............. 52

Figure 5-11 Voltage profile at node SX3029498C-Phase 1 during off peak hours.................... 52 
Figure 5-12 Loss Analysis of section during peak pricing hours....................... 53

Figure 5-13 Loss Analysis of section during off peak hours ........................... 54

Figure 5-14 KWh Losses for the section for various DR scenarios..................... 54

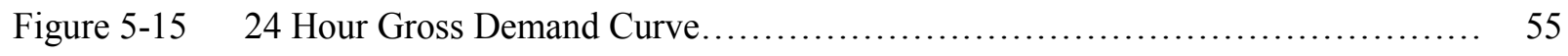

Figure 5-16 24 Hour Availability of Wind, PV and IPP resources .......................... 56

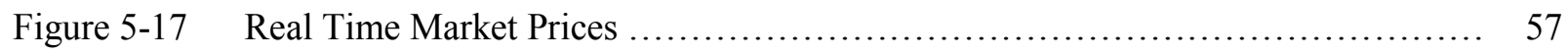

Figure 5-18 BESS Charge/Discharge Schedule ................................... 57

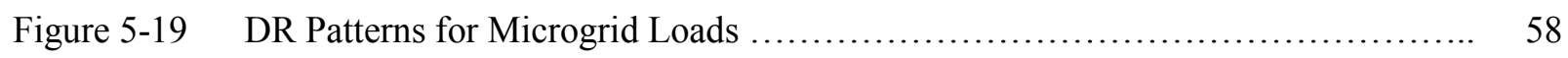

Figure 5-20 24 Hour Optimal dispatch schedule of DR, DERs and BESS ................. 58

Figure 5-21 Time series voltage analysis results of microgrid with optimal dispatch schedules............................................................. 62

Figure 5-22 8500 Node Test Feeder modeled as Microgrid............................... 63

Figure 5-23 24 Hour Availability of Wind, PV and IPP resources -8500 Node Microgrid........ 63

Figure 5-24 24 Hour Optimal dispatch schedule of DR, DERs and BESS for 8500 Node 64

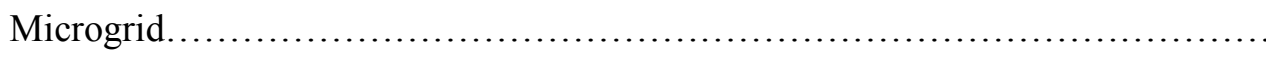

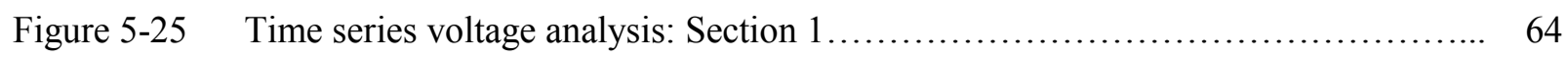

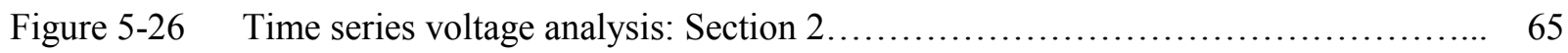




\section{LIST OF TABLES}

Table 3-1: $\quad$ Hourly Day Ahead RTP with Self Elasticity Coefficients $\ldots \ldots \ldots \ldots \ldots \ldots \ldots \ldots \ldots . \quad 25$

Table 3-2: $\quad$ PEM of Long Range Consumer ....................................... 28

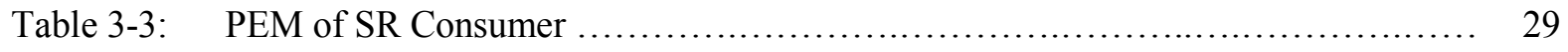

Table 3-4: $\quad$ PEM of RW Postponing Consumer ........................................ 30

Table 3-5: $\quad$ PEM of RW Advancing Consumer...................................... 31

Table 3-6: $\quad$ PEM of RW Mixed Consumer ........................................... 32

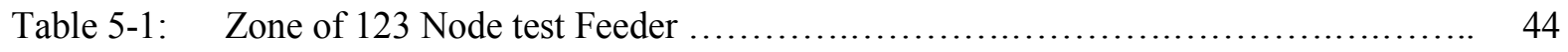

Table 5-2: $\quad$ Demand Side resources and their Bid Prices ............................... 55

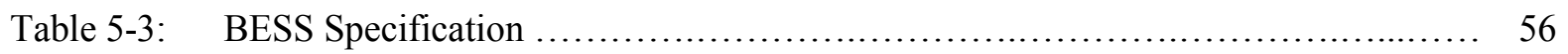

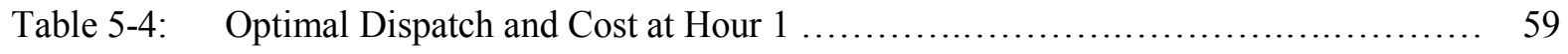

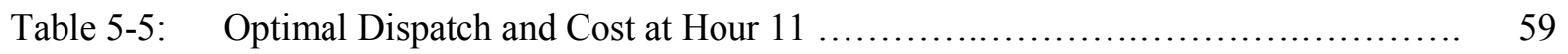

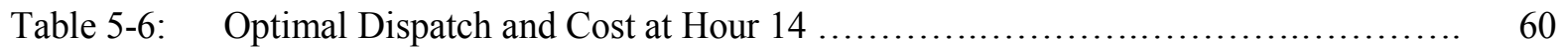

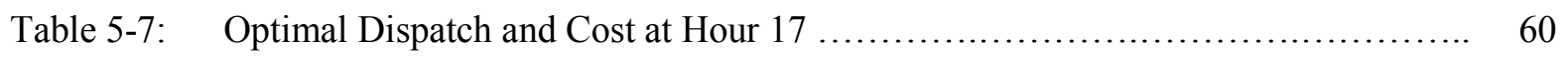

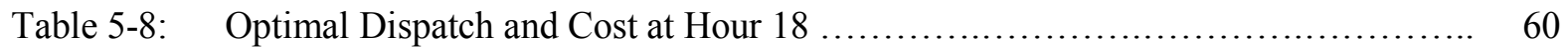

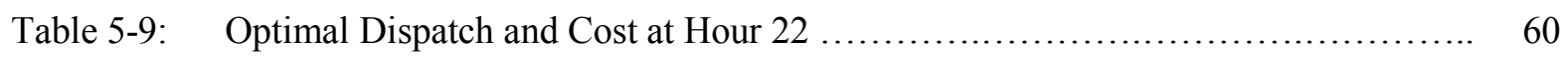




\section{NOMENCLATURE}

\begin{tabular}{|c|c|}
\hline DR & Demand Response \\
\hline BESS & Battery Energy Storage System \\
\hline DG & Distributed Generation \\
\hline DER & Distributed Energy Resource \\
\hline PEM & Price Elasticity Matrix \\
\hline VVC & Volt/Var Control \\
\hline DSB & Demand Side Bidding \\
\hline LP & Linear Programming \\
\hline IPP & Independent Power Producer \\
\hline LSE & Load Serving Entity \\
\hline RTP & Real Time Pricing \\
\hline AMI & Advanced Metering Infrastructure \\
\hline LR & Long Range \\
\hline SR & Short Range \\
\hline RW & Real World \\
\hline $\mathrm{SOC}$ & State Of Charge \\
\hline DOD & Depth Of Discharge \\
\hline DSS & Distribution System Simulator \\
\hline DMS & Distribution Management System \\
\hline
\end{tabular}




\section{Chapter 1}

\section{INTRODUCTION}

\subsection{Background}

The evolution of the deregulation trend in electricity market has led to the division of integrated power system into individual fields: generation, transmission and distribution. The deregulation has created a healthy competition in the distribution market among generation, transmission and distribution companies (Discos). The impacts of deregulation of electricity market have been far reaching beyond expectation. The most important among them is the modernization of the electric grid into what is now being termed as the Smart Grid.

A smart grid may be defined in a number of ways. In simple words, smart grid is an electric grid that employs monitoring, control, communication, and self-healing capabilities to efficiently deliver reliable, economic, and sustainable electricity services. Some of the features that characterize a Smart Grid as described in [1] are as follows:

1. Deployment and integration of demand response, demand side resources and energy efficiency resources.

2. Deployment and integration of distributed resources and generation, including renewable resources.

3. Deployment of smart technologies for metering, communications concerning grid operations/status and distribution automation.

4. Adoption of Demand Side Management (DSM) techniques like volt/var control, voltage reduction, etc.

5. Deployment and integration of advanced electricity storage and peak-shaving technologies, including plug-in electric and hybrid electric vehicles, etc.

Almost all discos are in the process of integrating one or the other strategies listed above, into their distribution network to make it smarter, efficient and more reliable. 


\subsection{Demand Response - Definition and Types}

The California Energy Crisis in 2000-2001 [2] threw the electricity market into an energy emergency where the demand could not be met by the available generation. Various causes for the crisis were reported such as poor deregulation policies, economic boom and excessive consumption of electricity. Irrespective of the nature of cause of the California Blackout, it was established that certain level of consumer response in demand reduction could have resulted in tremendous fall in electricity prices. This mainly led to the popularity of the term called Demand Response.

US Department of Energy (DOE) defines demand response as: changes in electric usage by end-use customers from their normal consumption patterns in response to changes in the price of electricity over time, or to incentive payments designed to induce lower electricity use at times of high wholesale market prices or when system reliability is jeopardized. In simpler terms Demand Response means demand responding to generation. DR paves way for achieving the market balance between generation and demand. DR programs are divided into two basic categories: Incentive Based Programs (IBP) and Time Based Programs (TBP) [3]. IBP and TBP are divided into several subgroups as shown in the graphic below.

\subsubsection{Incentive Based DR Programs}

Among Incentive Based Programs direct load control and interruptible/curtailable service programs are more suited for smaller residential consumers. In direct load control, utilities have the authority to shed the consumer"s load during some critical hours of the day. In Interruptible/Curtailable Service, the utility usually controls the customer"s loads remotely to achieve the required level of load reduction. Usually Heating, Ventilation and Air Conditioning (HVAC) loads are remotely controlled by the utility. In both these DR programs customers receive incentives/discounts to participate. Other incentive based DR programs are usually more suited for industrial consumers. These contracts normally exist between the distribution utility and a set of large consumers and most of these schemes involve curtailing the load by consumers for a specified period of time and by a specified level of energy to reduce congestion in the network. Demand Biding DR encourages the customer to bid for the amount of demand reduction and if the bid price is lesser than the market price, it may be accepted by the utility. In Emergency Demand Response, the customer is paid incentives as per laid out contracts for shedding their load during emergency periods. In case of both demand bidding and emergency DR, customers may face penalty if they do not curtail the demand by the amount specified. 


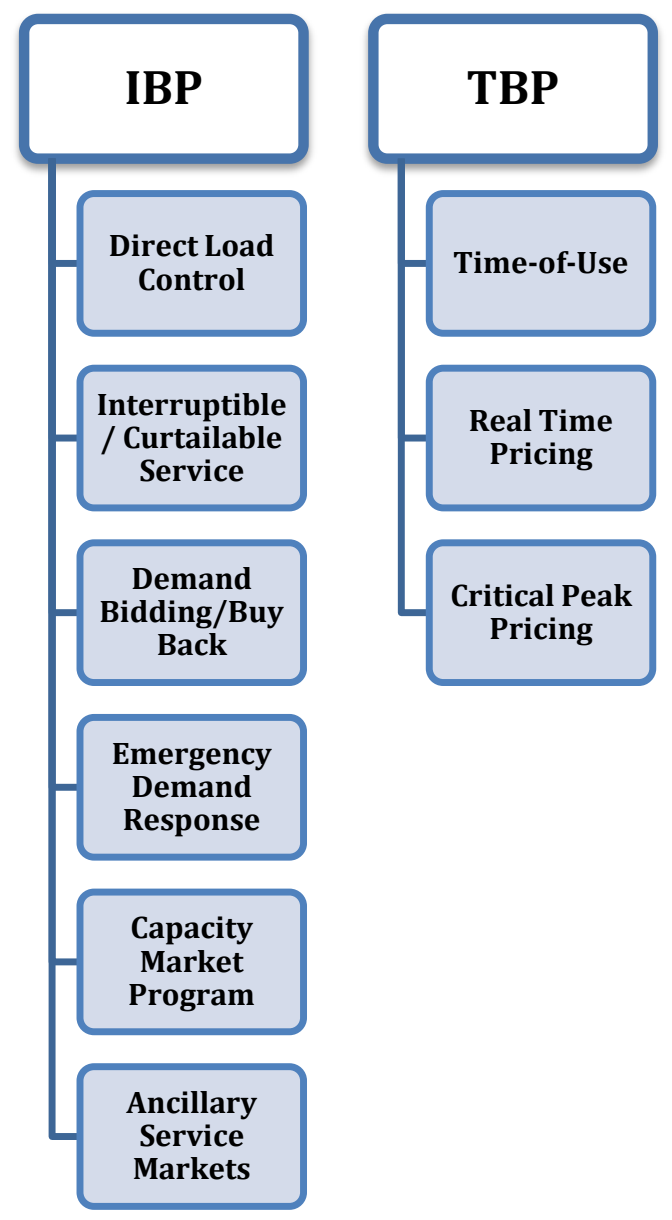

Figure 1-1: Types of Demand Response Programs

Capacity Market Programs are offered to customers who can curtail demand to pre-specified levels when system contingencies arise. Customers receive a day-ahead notice of events and are penalized when they do not respond to load curtailment signal. In Ancillary Services Market programs, customers bid for load curtailment in the spot market as an operating reserve. When bids are accepted, participants are paid incentive for committing to be on standby and are paid spot market energy price if load curtailment is required.

\subsubsection{Time Based DR Programs}

Time based DRs are more suited to residential consumers. In these schemes the day is usually divided into number of blocks that have different prices of electricity that reflect the true market price for generation of electricity. In case of Real Time Pricing (RTP), the day would be usually divided into a number of time slots, for example 24 one hour slots and each slot has a different price for electricity that reflects the real market clearing price. Time of Use (ToU) pricing is more or less similar to real time pricing, but with a fewer number of time slots to reduce the complexity involved with real time pricing. In 
most ToU schemes the day is divided into two pricing periods namely peak hours and off peak hours. Some utilities use three different pricing periods with a flat period included between the peak and the off peak periods. Peak periods will have higher tariff compared to off peak periods where the prices are comparatively lower. Critical Peak Pricing (CPP) has fixed rate tariff for most part of the day and high rate for consumption of electricity during a few pre-specified hours of the day.

\subsubsection{Day Ahead Real Time Pricing Programs}

RTP scheme engages maximum customer participation. Communicating real time prices to consumers and expecting them to respond would be cumbersome for both consumers and utility. So recently, utilities have laid down the Day-Ahead Real Time Pricing scheme wherein the next day's predicted real time prices would be sent to the customers before hand and they would be billed for their consumption based on the day-ahead price. For example, Ameren utilities, Illinois have incorporated the day ahead RTP scheme for residential consumers in its experimental program - Power Smart Pricing Program [4].

\subsection{Demand Response - Benefits and Challenges}

\subsubsection{Benefits}

DR provides multiple benefits to all the participants in the electricity market [5]. Figure 1-2 gives an overview of various benefits offered by DR.

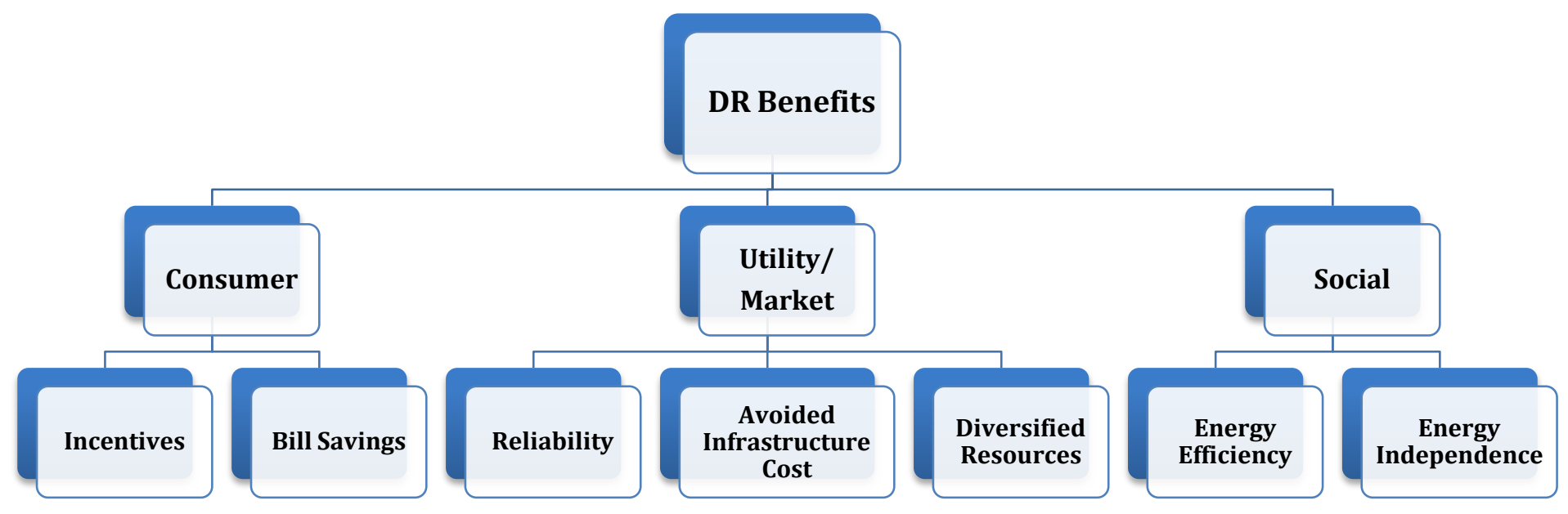

Figure 1-2: Benefits of Demand Response 
As seen in the figure, customers participating in DR programs can expect savings in electricity bills if they reduce their electricity usage during peak periods. Depending on the customer's readiness to curtail load during peak hours and their ability to optimize their load, customers can save electricity bills and earn incentives as well. Customers can also make use of the low prices during off peak (also called valley) periods to increase their energy consumption. This is an effective strategy for large industrial consumers who can increase their production without having to worry about the electricity prices. Distribution utilities can improve their reliability by smartly managing various DR schemes. Transmission and distribution companies can avoid purchasing bulk power from generating units at high prices and instead, can maximize the utilization of their existing resources and infrastructure with planned DR programs. These planned DR programs avoid building new infrastructure to meet the ever increasing electricity demand and thus all of the avoided or deferred costs will be reflected on the price of electricity for the consumers (DR programs participants and non-participants).

\subsubsection{Challenges}

Integration of DR into the US electric grid has gradually started moving from pilot projects into actual deployment. However, there are still certain barriers/challenges to DR deployment. These barriers can be grouped into three broad categories as follows:

1. Participation: Customer education is the most important challenge to the rapid deployment of DR programs.

2. Cost: The cost incurred by utilities (as incentives, for technology upgrade) and customers (technology upgrade) is another challenge that needs to be addressed.

3. Technology: Successful implementation of DR program lies in the installation of Advanced Metering Infrastructure (AMI) or smart meters at the customer premises. In addition, AMI's need to be enabled with fast two way communications to send signals between utility and consumer. This fundamental technological transfer is an immediate barrier for integration of DR on a large scale.

\subsection{Demand Side Resources}

As stated in section 1.1, one of the most important requirements for a smart grid is the deployment of energy efficient demand side resources. Distributed Energy Resources (DERs) or Distributed Generators (DGs) form the most important component of demand side resources. Following DGs, Energy Storage Systems (ESS) made their way into a smart distribution system owing to their ability to complement 
intermittent sources of renewable energy like wind and solar energy. DGs and ESS led to conceptualization of microgrids which enabled integration of demand side resources by means of intelligent communication and control techniques. At present, existence of microgrids has become an inherent part of a smart distribution network.

\subsubsection{Distributed Generators}

Distributed Generators refer to a range of small-scale and modular devices designed to provide electricity, and sometimes also thermal energy, located at the distribution side of the electric grid [6]. They include renewable energy technologies such as photovoltaic arrays, wind turbines, microturbines, reciprocating engines, fuel cells, combustion turbines, and steam turbines and combined heat and power systems. DG technology is not new to distribution network, until the smart grid era, DGs were mainly used to provide emergency standby power during outages to uphold continuity of supply to certain essential facilities such as hospitals and telecommunication centers [7]. With the emergence of advanced communication and control techniques, DGs play a far more vital role to address issues of grid security, reliability and energy efficiency. Small DGs at consumer premises have led to the new concept of consumer participation in the energy market. At the same time DGs have also posed technological challenges such as reverse power flow to the grid and further raised questions regarding net metering for consumers that deploy them at their premises. Modeling and analysis of DGs has become an inherent part of most smart grid research projects around the world.

\subsubsection{Battery Energy Storage Systems}

Recent developments and advances in energy storage technologies offer potentially viable solution for various smart grid applications, allowing both transmission and distribution system to be operated in a more flexible and controllable manner. Some of the applications of energy storage systems (ESS) in power systems are listed in figure 1-3 [8]. BESS, being identified as one of the most cost effective storage solutions, finds growing application in small scale distribution networks and microgrids. Similar to the case of distributed generators, BESS were earlier employed for fundamental power system operation namely spinning power reserve, load frequency control and emergency backup [9]. However, in the modern grid BESS at the residential level can support numerous smart grid goals, including peak shaving, load leveling, power factor correction and improved reliability [10]. So, the inclusion of BESS in modeling a modern distribution/microgrid system is another area of interest. 

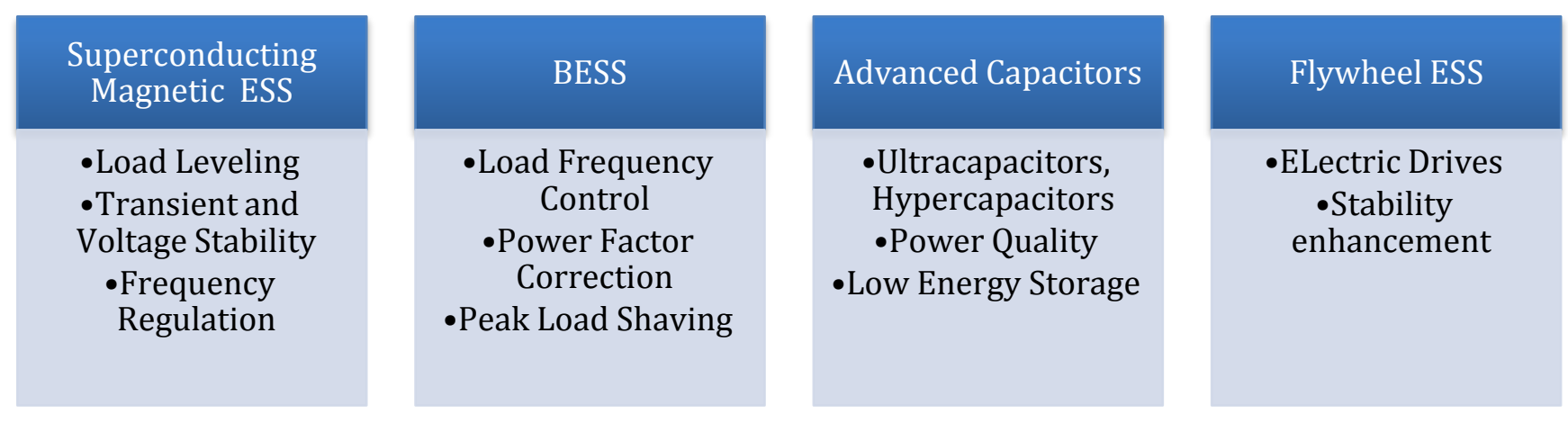

Figure 1-3: Types of Energy Storage Systems with Application

\subsection{Microgrids}

Another implication of deregulation and interactive electricity market is the evolution of microgrids. Microgrids are low voltage distribution networks comprising DGs, storage devices, and responsive loads that can operate either interconnected to the main grid or in an intentional island mode [11]. Coordinated operation of DGs and storage systems is the most important requirement of a microgrid. Tremendous research and development is in progress to develop a control strategy for the microgrid operation. A microgrid controller has to cater to all the technical challenges faced by the microgrid community and should also incorporate most of the smart grid features to improve the reliability and security of the microgrid. The controller has to optimize the microgrid operation during both grid connected and islanded operation [12].

\subsubsection{Microgrid Control Operation}

In addition to optimization of microgrid operation, the controller has to perform several critical activities which are common for any type of control architecture. Some of the key issues to be taken care of by the controller especially during islanded mode of operation are as follows [13].

a. Voltage and Frequency Control: During islanded operation the primary or intermediate energy sources have to be controlled to adjust voltage and frequency to match the operation of loads connected in the microgrid. In worst case, the load should be disconnected by the controller.

b. Supply and Demand Balance: This is more of an optimization function during grid connection operation. However in the islanded mode, available storage should be used. In addition, the 
controller should incorporate demand response events during this phase of operation to ensure reliability and security of the whole or part of the system.

c. Power Quality: Power quality should be maintained for microgrid in islanded operation with sufficient supply of reactive energy to shrink voltage sags. The energy storage devices should react rapidly to frequency and voltage change by charging/discharging.

d. Communication among Microgrid Components: Coordination of DGs, storage and responding loads should be established in a microgrid through advanced communication infrastructure.

e. Planned Microgrid Islanding: Planned islanding should be possible for a microgrid to maintain the continuity of supply during planned outages, like substation maintenance.

\subsubsection{Microgrid Controller Architecture}

Even though numerous control strategies are being developed for microgrids, they can broadly be classified as - Centralized Control Architecture and Distributed Control Architecture.

The prominent features of centralized control architecture are hierarchical architecture consisting of the Distribution Management System (DMS), the Microgrid Central Controller (usually referred to as Master Controller or MGCC), the Microsource (any source of power within a microgrid) Controllers (MC) and the Load Controllers that are locally located around DGs and loads respectively. Microsource Controller uses local information to control the voltage and the frequency of the microgrid in transient conditions. MCs coordinates with the central controller, when connected to the power grid, and perform local optimization of the DG active and reactive power production, and fast load tracking under an islanding situation. Local load controllers at controllable loads provide load control capabilities on receiving signals from the MGCC for load management.

The distributed control architecture has been gaining even more attention since it has got many advantages realized by the use of Multi-Agent Systems (MAS). Adopting a distributed control strategy with MAS provides many benefits. The semi autonomous feature of MAS suggests that the agents take decisions driven by their behaviors without a central controller. In addition, when required, agents can communicate with other agents before taking a decision or performing an action. The agent has knowledge only of the status of the unit it is controlling. However, it can update its own data table of neighboring systems by repeatedly communicating with other agents. This avoids a lot of confusion that the microgrid master controller has to go through. Another important benefit is the cost factor. Provided that most microgrids are small communities comprised mostly of small scale DGs, a centralized control would prove to be very expensive. On the other hand, MAS has always been less expensive and can be 
implemented by a set of microcontrollers or wireless sensor networks. Another notable feature is that agents can update and adapt themselves to any change in the system which will be really effective in microgrids for "plug and play" feature of generating units [14].

\subsubsection{Energy Management in a Microgrid}

Energy management is another important facet of any microgrid network. To ensure a quality power management scheme numerous factors need to be considered namely communication infrastructure, type and number of controllers, control algorithm, balancing of multiple DGs, loads and dynamics of the microgrid system, etc. Responsive load is becoming an integral part of most microgrid. Modern microgrids aim at achieving an interactive energy market where the consumers participate and bid into the market, often termed as Demand Side Bidding (DSB). The design of a power management scheme begins with defining the attributes and objective of the microgrid system which can be listed as follows [15].

- Meet consumer load demand within voltage and frequency limits.

- Reduce line losses, provide VAR support, and stimulate power market opportunities for trading ancillary services to the utility grid.

- Ensure stable and adaptable control system behavior for seamless "plug-and-play" implementation and sensible load control.

- Minimize economic factors (e.g. fuel costs, operation and maintenance, etc.)

- Minimize environmental impact (e.g. emissions)

\subsubsection{Microgrid Modeling and Simulation}

Modeling and Simulation of microgrid is an area where tremendous amount of research is being done. Development of a simulation platform, setting up virtual microgrids on a laboratory setup, developing models for DGs and storage units are the keys to develop successful microgrid architectures. Numerous prototype models have been developed and simulated in different tools. In particular a lot of progress has been made in the modeling and simulation of the following.

- Photovoltaics

- Wind Turbine

- Micro Turbine

- Battery Storage

- Responsive Load 
Even Though several models are available, there are no set standards for these models for researchers to work on and develop their control strategies.

\subsection{Demand Side Bidding and Load Serving Entities}

Responsive load is an integral part of the modern day grid. Smart grid in general and microgrids in particular aim at achieving an interactive energy market where the consumers participate in market operation for the success of various DR programs that can lead to the benefit of both the utility and the consumers. This has led to demand side bidding where in the consumer participates in the competitive market like any other supplier. This kind of bidding strategy can prove effective even in a microgrid market provided there is a central microgrid controller that settles the bids between DGs/ Independent Power Producers (IPPs) and consumers. So far, only large industrial consumers have been seen as potential participants in DSB due to their ability to shed considerable MW demand during peak hours. However, in a microgrid paradigm, where the generation and demand levels are relatively low (in the range of a few hundreds of KWs), there is a good potential for residential consumers to participate in the market bidding. This can be achieved by load serving entities (LSEs) or DR aggregators (these entities are also being named as DR contractors or simply aggregators). LSEs can bid into the open microgrid market for the volume of DR that can be achieved by them and the market clearing optimization should then consider these bids as similar to generation bids received from IPPs. For the successful implementation of DSB, LSEs are in need of comprehensive DR models.

\subsection{Problem Statement}

In this thesis a comprehensive model for demand response is developed by utilizing consumer behavior modeling considering different scenarios and levels of consumer rationality. The developed DR model is integrated into distribution power flow of large real world distribution networks. The effect of DR on voltage profile and losses of the system has been analyzed. Based on the results obtained, recommendations have been made for considering DR as a parameter for distribution side colt/var control for benefits of peak load shaving and energy efficiency.

Further, a market optimization model is developed for an islanded microgrid that includes various smart grid elements namely DR, DERs and BESS. A charge/discharge model for BESS has been developed and integrated into the optimization program. LSEs are included in the double sided microgrid energy market and demand side bidding by LSEs is incorporated by utilizing the DR models developed. The optimization program uses linear programming technique to determine the dispatch schedule of DERs, 
BESS and the level of DR to minimize the operating cost of the microgrid market. A steady state voltage analysis has been performed on a test microgrid system to show the feasibility of the proposed market optimization.

A few assumptions made in this thesis work are listed below,

- $\quad$ AMI or smart meter is assumed to be deployed at the customeres premise to facilitate day ahead real time pricing signal.

- Elasticity coefficients (introduced later in chapter 3) used to model DR have been either inferred from available data or extrapolated from previous works. Extensive research is going on to develop sophisticated regression models for computing these elasticity coefficients which is beyond the scope of this work.

\subsection{Approach}

The sections to follow will model consumer demand response and a microgrid energy market including LSEs, BESS and DGs. The key aspects of this research are highlighted below in subsections below.

\subsubsection{DR Modeling}

- Consumer behavior is modeled by applying economic principles to electricity demand - price relationships.

- Consumer rationality is translated into Price Elasticity Matrices (PEM) composed of self elasticity and cross elasticity coefficients.

- DR model is derived from PEMs using mathematical computations.

- DR model is integrated into time series power flow for large test feeders, modeled in distribution simulation tool OpenDSS.

- DR patterns for loads are generated by establishing a communication interface between OpenDSS and Matlab in real time.

\subsubsection{Microgrid Market Optimization}

- BESS is modeled and its charge/discharge schedule is determined over 24 hour period.

- Optimization is achieved through linear programming in Matlab.

- LP considers bids from all the participants of the microgrid namely DGs, BESS and LSEs 
- Optimized dispatch schedule is determined by LP for minimizing operating cost of the microgrid.

- The feasibility of the proposed optimization strategy is determined by simulating unbalanced 3phase power flow for a microgrid test system.

\subsection{Outline}

The outline of the remaining chapters is given in this section.

Chapter 2 is a comprehensive literature review on demand response, history and evolution of DR, various approaches to model DR, industry experiences with DR and other works that implement DR as a smart grid measure with different objectives. A literature review on microgrids and energy management in microgrid with demand side resources is also included.

Chapter 3 is about modeling of demand response and mathematical formulas used for obtaining the models. The microgrid market optimization problem is formulated with the developed models of demand response and battery energy storage systems.

Chapter 4 gives a detailed description of the software packages used in this research. The advantages offered by the software to the relevant applications are also discussed.

Chapter 5 presents the test systems, simulations for analyzing demand response and various test scenarios used for microgrid market optimization with results.

Finally, chapter 6 lists conclusion of this study and provides scope for future work. 


\section{Chapter 2}

\section{LITERATURE REVIEW}

This chapter reviews the literature for demand response and microgrid market.

\subsection{Demand Response - History and Evolution}

The evolving platform for DR was set way back in the early 1980s when spot pricing was introduced for the first time as a new concept of electricity pricing [16]. Effect of spot prices on line losses, quality of supply and reactive energy are also highlighted in the paper. Also, a mathematical theory was formulated for optimum spot pricing with social welfare objectives that also takes into account a model for the participants of the spot pricing market namely generating firms and consumers. Reference [17] provides substantial literature on fundamental principles on spot pricing of electricity and economic analysis of spot pricing. The authors have laid a theoretically strong foundation on establishing utility - consumer interaction for the benefit of both parties and the electricity market. Tin [18], he authors have also provided a detailed evaluation on the variable costs of electric energy on an hourly basis using the theory of spot prices. In this work, the spot prices were decomposed into six basic components such as fuel cost, generation cost, network maintenance cost, losses cost and quality of supply cost. Further with annual network data, spot prices for the entire year were determined.

\subsection{Demand Response and Consumer Behavior Modeling}

The evolution of spot electricity prices was followed by a series of works in the area of consumer behavior modeling assuming spot pricing of electricity. In [19], the authors A.K. David and Y.Z. Li have elaborated the importance of consumer behavioral models for developing analytical models for electric systems with dynamic real time pricing. The consumer behavioral model developed in the paper relies on the concepts of demand elasticity across time, consumer economic rationality and also depends on the price formation model at the supply side. The consumers were categorized as long range, short range and real world consumers based on their rationality. However, constant values of demand price elasticities (self and cross elasticities) were assumed in this work, that questions the level of accuracy of the developed price elasticity matrices. This particular limitation of this work formed a major source of motivation for this thesis work to develop more accurate price elasticity matrices for different consumer categories. Another work by the same authors [20] includes the issues of optimal response of industrial 
consumers and the effect of large scale penetration of real time tariffs on the utility load curve. Further in this work, the authors have characterized spot pricing and its effect on load response as a short term load and price forecast problem where demand and price are interrelated and interdependent entities. In yet another work, these authors have proposed a real time optimal power flow algorithm that computes the real time price signals for the market using fixed demand curves [21]. These demand curves are then adjusted according to the demand price elasticity data of each consumer. The algorithm was applied to a test system with assumed values of price elasticities (again with the same limitation in PEM). The performance of the iterative OPF algorithm with day ahead and spot prices were analyzed for a particular consumer type. However the scope of spot pricing was very limited at that time owing to the integrated nature of power systems and lesser flexibility among the end-users.

\subsection{Load Forecast with Demand Response}

With demand response, the loads tend to change and so does the forecasted data based on historical behavioral pattern of the distribution system. The load forecasts were based on price insensitive historic load data of the regulated era. However, it is expected that a majority of consumers would shift their loads to less expensive hours of the day in order to avoid higher costs. In recent years, short term load forecast (STLF) tools based on artificial neural network, fuzzy logic inference, regression models and other techniques have received wide acceptance in the electric utility industry owing to their accuracy and fast forecasting. One of the complicating factors in adopting current STLF tools to a price sensitive environment is the price responsive load behavior and another is the lack of historic data [22]. The relationship between real time price and load behavior is complicated. Price varies with demand and consecutively, demand influences the fluctuation of price. Price, in turn influences the behavior of consumer load. So there is a mutual dependence.

Efforts are underway for developing accurate load forecasting techniques in a price sensitive environment. In an early work [23], an autoregressive integrated moving array (ARIMA) load forecast model was developed and then it was conditioned for price sensitivity using PEM. In some of these methods [24], [25], load forecasting is done for the normal price insensitive case using artificial neural network and then the effect of real time pricing is incorporated using fuzzy logic and a set of non linear constraints. In this technique, the idea is to first predict the load with existing artificial neural network technique that produces a price insensitive load forecast. The required change to this forecast is then carried out by a fuzzy logic module that responds to price signals. 


\subsection{Demand Response and Smart Grid}

In the smart grid paradigm, the spot pricing has evolved into a multifunctional, multi-objective concept namely, demand response. In most of the recent research works, DR has been employed for a number of potential benefits that it offers as explained in section 1.3.1. Significant work has been done on developing DR models considering different types of pricing schemes and scenarios. A framework for incorporating DR in a competitive market condition has been laid in [26]. Demand redistribution matrix has been used to model load reduction and load recovery patterns of industrial consumers. Further, demand side bidding was introduced and optimization techniques were used to match generation with load in real time. In these works, a demand redistribution pattern for demand bidders was incorporated instead of the demand price elasticity matrix to account for the shift in their load during peak hours. Reference [27] further builds on the demand redistribution matrix and proposes a novel concept for day ahead generation scheduling incorporated as a mixed integer problem. It was concluded that unreasonable demand bidding may cause unexpected problems in electricity market. So proper strategies have to be formulated by the consumer while bidding into the real time market for demand response. In [28], PEMs were revisited and used in a generation scheduling program that also uses the elasticities to compute real time market prices on a half hourly basis over a period of 24 hours. An optimized PEM will yield lower real time marginal prices. This work was further extrapolated in [29], where DR was introduced into a perfectly deregulated market structure. A wholesale bidding mechanism involving ISO, utility and the consumer was proposed that uses consumer modeling through price elasticity matrices considering load shifting by consumers throughout the auction time-frame. The proposed algorithm uses both unit commitment and economic dispatch in the presence of elasticity data to determine market price. The algorithm also lets participants submit their bids in a compact PEM which was a significant improvement from previous works.

Another area of smart grid research focuses on the implementation of DR at the household level. One such work takes into account the intelligent home appliances that can be smartly controlled using thermostat to consume less energy during peak hours [30]. In this work, the power consumption pattern of residential heating ventilation and air conditioning (HVAC) system is modeled. DR model for HVAC was made compatible with the distribution automation via. Implicit channel (DAVIC) framework. On similar lines, [31] has attempted to mathematically determine the thermal comfort zone of residential consumers

using HVAC systems. The mathematical model is used in an optimization problem that maximizes profit of a retailer by determining the optimized incentives to be paid to the consumers. The authors have 
applied this DR model on a small test system to show how retailers can stabilize their profit levels during peak hours of highly fluctuating market price.

Intelligent DR models have been developed in some works that were incorporated into the utility distribution management system (DMS). In [32] the authors have developed a working DR engine based on expert systems suitable for large industrial customers. The proposed DR model consists of a utility DR engine that computes DR (load reduction and price) signals based on network model, load forecasting data and market rates. This DR signal is then communicated to the customer DR module that sends DR commands to the AMIs of participating consumers. The model has been developed in Matlab Simulink. Further, in [33] the authors have developed a comprehensive event based DR architecture that can be integrated with the DMS at network control center level. The proposed DR architecture has also highlighted the role that an aggregator can play in establishing DR contracts between utility and smaller consumers. The authors have taken into account various levels of communication between end-user, aggregator and utility. In this work too, the DR engine functions from the distribution utility level and generates the necessary DR signals for the customer AMI. This work also sheds light on other important aspects for DR consideration namely AMI technology, data models and communication standards, DR messages and DR validation technique.

Recently, there has been a lot of emphasis on developing DR model considering the distribution network parameters in order to coordinate DR with the other Demand Side Management (DSM) programs. One such literature [34] proposes a novel concept of considering DR as a parameter for applying volt/var control (VVC) on a real time basis to realize the maximum potential of both these DMS measures. According to the authors the availability of real time distribution operation model by means of Advanced Metering Infrastructure (AMI) and advancements in Distribution Automation (DA) and communication standards can help integrate DR with volt/var optimization in a systematic way. In [35], the authors have further demonstrated the relationship between DR and VVC. The impact of DR on the results of coordinated Voltage and Var Optimization was analyzed by applying DR at different locations along distribution feeder:

- Close to the substation bus

- In the middle of the feeder

- Close to the end of the feeder

The authors have also pointed out the benefits of DR with service restoration and feeder reconfiguration. It was concluded that to best utilize the benefits of DR, the distribution automation and DMS algorithms 
need to consider the near real time operational model of the distribution network, which constantly changes with DR penetration in the system.

\subsection{Microgrids and Distributed Energy Resources}

\subsubsection{Practical Demonstrations of Microgrid}

Practical demonstrations of microgrid around the world provide us with a tremendous volume of information on its implementation, control strategies, energy management strategies and modeling and simulation techniques incorporated [36]. Some of the important microgrid demonstrations are described in brief.

\subsubsection{The EU Microgrid Research Project}

This project included a consortium led by National Technical University of Athens and partners from seven EU countries including utilities, manufacturers and other universities. The objective of this project was to study the operation of microgrids, to increase penetration of renewables and other DERs while reducing carbon emissions during interconnected and islanded modes. It is also aimed to defining and developing control strategies to ensure efficient, reliable and economic operation and management of microgrids and to include appropriate protection and grounding policies to assure safety, fault detection, separation and islanded operation. The EU demonstration sites included Greece: The Kythnos Island Microgrid, Netherlands: Continuon's MV/LV facility, Germany: MVV Residential Demonstration at Mannheim-Wallstadt.

\subsubsection{CERTS Microgrid}

This well-known U.S. microgrid RD\&D effort has been pursued under the Consortium for Electric Reliability Technology Solutions (CERTS) [37]. Some of the salient features of the CERTS Microgrid are:

a. A lack of fast electrical controls where in the operation of generators is controlled locally by power electronic devices incorporating droop characteristics that respond to locally monitored frequency and voltage.

b. A single point of common coupling (PCC), and does not export. This means that to the utility the microgrid appears as a single controlled load that is no different than customers. 
c. A dispersed plug-and-play system. No custom engineering is required for interconnection of any single device, as long as it has CERTS microgrid capability, making system configuration flexible and variable.

\subsubsection{Microgrid Research Activities in Japan}

\section{NEDO Microgrid Projects}

The New Energy and Industrial Technology Development Organization (NEDO) field tests focus on the integration of new energy sources into a local distribution network [38]. Proposed microgrid projects in Aomori, Aichi, and Kyoto since they have a significant renewable energy component.

- The Aomori Project in Hachinohe: A central feature of the system is that only renewable energy sources, including PV, wind turbines (together totaling $100 \mathrm{~kW}$ ), and biomass, are used to supply electricity and heat. The controllable DERs consist of three $170-\mathrm{kW}$ generator sets $(510 \mathrm{~kW}$ total) burning sewage digester gas, a 100-kW lead-acid battery bank, and a 1.0-t/h woody biomass boiler.

- The Aichi Project near the Central Japan Airport: The main feature of this project is a combination of the following fuel cells as the main sources: two $[270-\mathrm{kW}$ and $300-\mathrm{kW}$ molten carbonate fuel cells (MCFCs)], four 200-kW phosphoric acid fuel cells (PAFCs), and a 50- kW solid oxide fuel cell (SOFC).

- The Kyoto Project at Kyotango: This is the first virtual microgrid demonstration project covering a 40-km span called the Kyoto Eco Energy Project, which started operation in December 2005. It incorporates the following generation capacities: $50 \mathrm{KW}$ of PV, $50 \mathrm{KW}$ of WTs, $5 \times 80 \mathrm{KW}$ biogas

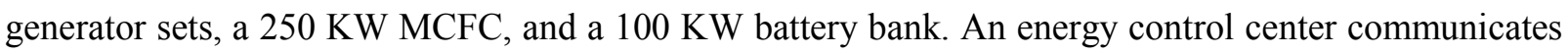
with the DERs by internet protocol over the legacy telecom network to balance demand and supply, and energy is fed into the legacy distribution system.

\subsubsection{Microgrid Energy Management}

The concept of microgrids has posed several questions on energy management issues such as:

1. What kind of energy management strategies need to be followed for a microgrid?

2. Who is responsible for clearing the microgrid market during its islanded mode?

3. How do IPPs and consumers participate in the microgrid market? 
A good amount of literature is available because of growing interests in the areas of microgrid energy management. In [39]-[41] authors have laid out the rules for setting Market Clearing Price (MCP) for a microgrid Energy Management System (EMS) in the competitive electricity market where the Microgrid Central controller (MCC) participates in the bidding process to finalize pricing mechanism. Micro turbine, fuel cell, diesel, wind and PV generators also bid in the microgrid market. MCP was deduced for two different cases: DGs bidding with fixed demand and both DGs and consumers bidding in microgrid market. Also, day-ahead and real time market prices have been considered for market clearing. Reference [42] demonstrates various functions of a central microgrid controller during its participation in real time market in grid connected mode. The optimization functions of the MGCC consist of economic scheduling, forecasting (short term) for electrical load, and renewable energy sources, security assessment and demand side management. However, the literature does not include DR in the DSB process for solving the optimization problem.

\subsection{Battery Energy Storage System}

Another important aspect of the modern day microgrid is storage. General introduction on types of storage and their application to power systems has been discussed in section 1.4.2. Battery Energy Storage Systems are of special interest in microgrid application owing to their special features such as bulk energy storage densities, greater cycling capabilities, higher reliability rapid charge/discharge capabilities and lower costs.

\subsubsection{Early Works on BESS}

Research on BESS has dated far back to early eighties. In [43] the author used levelized incremental cost method to simulate the hourly operation of a central BESS to be included in unit commitment and economic dispatch routines and other applications that use storage. The algorithm matches generation and storage in a way that it levelizes the incremental cost of generation over a predefined period of time. The impact of BESS on peak shaving and regulation aspects of power system dispatch was evaluated in [44]. Battery and power electronic models were developed and integrated into a hybrid power simulator to dispatch various generator units in combination with BESS to meet hourly loads. The application of BESS on frequency regulation of a microgrid type islanded system was proposed way back in 1993 [45]. The authors had analyzed the operation of the large BESS through a series of computer simulations and recommended that BESS reduces drastically the frequency deviations resulting from sudden demand variations. In [46] the authors have developed an algorithm combining multi-pass dynamic programming (MPDP) with a time-shift technique for determining economic dispatch of BESS and optimal BESS 
power and energy capacities in a power system. In addition, applications of BESS for load leveling, peak shaving and load following were demonstrated.

\subsubsection{BESS in Microgrid}

Application of BESS in microgrid is an area that is gaining a lot of research interest. Some researchers have already started exploring the possibilities of integrating BESS into microgrid for energy management and control applications. In [47], a formulation for determining optimal operating strategy and cost optimization scheme as well as reducing the emissions of a microgrid has been presented. The authors have applied a multi objective optimization environmental economic problem of the microgrid. A simplified BESS model constrained by its State Of Charge (SOC) was considered as one of the components of the microgrid. A Composite Energy Storage system (CESS) that contains both BESS and ultracapacitor was proposed in [48] to suppress the effects of intermittencies of renewable energy sources such as PV in a microgrid. The proposed CESS based DC-DC converter was used for energy management of the microgrid with intermittent sources. Similarly [49] utilizes a battery supercapacitor hybrid storage (BSHS) module which is composed of batteries, supercapacitor module, BUCK/BOOST bi-directional converter and voltage sources converter to mitigate the intermittencies caused due to PV and wind turbine generators Reference [50] manages the SOC of a BESS in a microgrid. The authors have proposed and demonstrated a controller that accomplishes the task of controlling the energy levels of the BESS to within an acceptable range. In [51] a framework is proposed for a microgrid, such as for a colony or small township of homes that generate electric power from solar energy and use it directly when possible, and from stored battery power at other times. The paper also uses auction theory to determine the most efficient and competing pricing mechanism in the proposed microgrid system model in the presence of $\mathrm{PV}$ and BESS.

\subsubsection{BESS - Optimal Schedule with Variable Tariff}

A number of recent studies have investigated the optimal operation of BESS in the electricity market. In [52], a dynamic programming algorithm was developed to determine the optimal charge/discharge schedule of BESS. The work also determines maximum payoff for BESS application namely load redistribution and peak load shaving while accounting for charging rate, battery voltage fluctuation and internal losses as a function of charge state considering two different pricing contracts namely bi-period and tri-period pricing. Under a utility real time pricing scenario, the spot price of electricity will directly influence the revenue that the BESS can generate with its charge/discharge schedule. In [53], a multi-pass 
iterative particle swarm optimization algorithm is used to solve the optimal operating schedule of a BESS for an industrial time-of-use rate user with wind turbine generators.

In both the above works, only time of use rates were considered while determining the optimum schedule for battery. In [54] the authors have investigated optimal operation strategy for a BESS in a day ahead spot pricing market in order to achieve the maximum profits. The authors have used sequential quadratic programming method to determine the optimum charge/discharge schedule of the BESS with certain limitation on the energy storage and charge/discharge capability of the battery. The payback times for two different battery storage technologies have been compared. 


\section{Chapter 3}

\section{MATHEMATICAL MODEL AND FORMULATION}

\subsection{Modeling of Demand Response}

\subsubsection{Price Elasticity Matrix - Fundamentals}

By far, PEM has been the most powerful and feasible way of modeling consumer behavior for DR. Considering electricity as any other commodity, electricity demand does change with price. The demand price elasticity is defined by the following equation.

$$
E=\frac{\Delta d / d_{0}}{\Delta p / p_{0}}
$$

Where $\Delta d$ and $\Delta p$ are the changes in demand and price respectively and $d_{0}$ and $p_{0}$ are the base demand and price respectively.

Equation 3.1 is the basis for developing PEMs. Elasticity is composed of two different coefficients namely self elasticity (or own-price elasticity) and cross elasticity. Self elasticity (equation 3.2) is defined as the change in demand at a time instant, $\mathrm{t}_{i}^{\text {ee }}$ due to change in price at the same time instant, $\mathrm{t}_{\mathrm{i}}^{\text {ee }}$. Since change in price will have an inverse effect on change in demand, self elasticity takes a negative value. Also there is a cross-time effect involved in the time varying demand-price elasticity. Cross elasticity (equation 3.3) is defined as the change in demand at time instant, $\mathrm{t}_{\mathrm{i}}{ }^{\text {ee }}$ due to change in price at some other time instant, $\mathrm{t}_{j}^{\mathrm{e}}$. Cross elasticity will be either positive or zero depending on whether the customer is willing to shift the load or not.

Self Elasticity Coefficient

$$
E(i, i)=\frac{\Delta d\left(\mathrm{t}_{\mathrm{i}}\right) / d_{0}}{\Delta p\left(\mathrm{t}_{\mathrm{i}}\right) / p_{0}}
$$


Cross Elasticity Coefficient

$$
E(i, j)=\frac{\Delta d\left(\mathrm{t}_{\mathrm{i}}\right) / d_{0}}{\Delta p\left(\mathrm{t}_{\mathrm{j}}\right) / p_{0}}
$$

Self elasticity is a measure of load curtailment by the consumer where as cross elasticity is a measure of load shifting. Both these constituents put together form the concept of DR.

For a RTP scenario with hourly varying rates, PEM is of the order $24 \mathrm{X} 24$. The diagonal elements of the PEM represent self elasticity coefficients and the off-diagonal elements represent cross elasticity coefficients. Each column of a PEM represents the scheduling of loads throughout the day owing to the change in price at the time instant corresponding to the column number. The overall change in load at time, $\mathrm{t}_{\mathrm{i}}{ }^{\mathrm{e}}$ due to change in price throughout the day can be obtained by summing up the entire row corresponding to , $\mathrm{t}_{\mathrm{i}}{ }^{\mathrm{e}}$ as shown in equation 3.4.

$$
\Delta d\left(t_{i}\right)=\sum_{j=1}^{24} E(i, j) *\left(\Delta p_{j} / p_{0}\right) * d_{0}
$$

The optimum model of PEM is the key to determine the level of DR and load reduction or redistribution possible per customer. With the availability of AMI and other Distribution Automation (DA) features, the real time distribution operation model with DR data can be utilized for identifying the exact set points for volt/var control.

Earlier works involving PEMs have assumed constant values of self and cross elasticity coefficients which may not be applied in a real world scenario. With a constant self elasticity coefficient, for example, the relative effect on consumption is the same for an increase in price per KWH from $\$ 0.01$ to $\$ 0.02$ as an increase in price from $\$ 0.05$ to $\$ 0.10$ which is not the case with any rational consumer. These varying self and cross elasticity coefficients served as a guideline for framing the PEMs in this work.

\subsubsection{Consumer Rationality Assumptions}

Consumer rationality assumptions indicating real world scenarios have been utilized to develop DR models. Consumers were grouped into five different categories where any real world consumer should fall into one of these categories. Consider a utility RTP curve over a 24 hour period on a summer peak 
day [55] as shown in table 3-1. The PEMs developed correspond to this summer peak day RTP and should be applied to calculate DR only for the summer peak days. With historical data on RTPs for various seasons and corresponding consumer load shapes, PEMs for different seasons can be developed. Constant values of self elasticity coefficients are assumed for extremely low prices. Beyond the threshold of 3 cents per KWH, self elasticity increases with increasing price signal. These self and cross elasticity coefficients have been derived from huge volume of price and self elasticity relationship of consumer load profile for an entire year [55].

The five different consumer categories based on rationality assumptions are shown in figure 3-1.
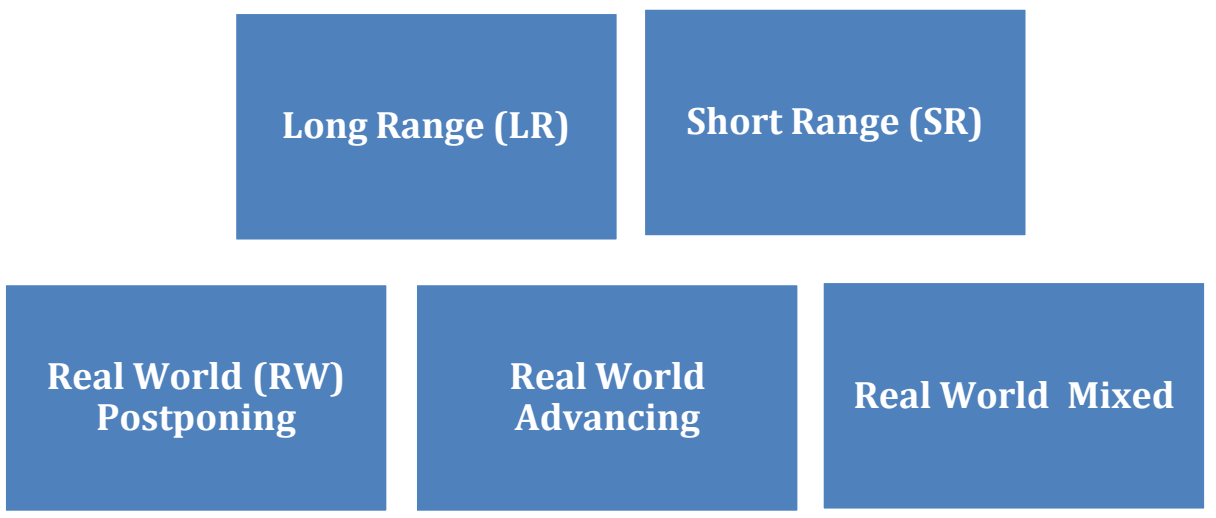

Figure 3-1: Types of Consumers 


\begin{tabular}{|c|c|c|}
\hline $\begin{array}{l}\text { Hour } \\
\text { of } \\
\text { Day }\end{array}$ & $\begin{array}{l}\text { Price } \\
\text { (Cents } \\
\text { per } \\
\text { KWH) }\end{array}$ & $\begin{array}{l}\text { Self } \\
\text { Elasticity }\end{array}$ \\
\hline 1 & 2 & -.01 \\
\hline 2 & 2 & -.01 \\
\hline 3 & 2 & -.01 \\
\hline 4 & 2 & -.01 \\
\hline 5 & 2 & -.01 \\
\hline 6 & 2 & -.01 \\
\hline 7 & 3 & -.01 \\
\hline 8 & 4 & -.02 \\
\hline 9 & 6 & -.02 \\
\hline 10 & 8 & -.02 \\
\hline 11 & 10 & -.03 \\
\hline 12 & 10 & -.03 \\
\hline 13 & 11 & -.04 \\
\hline 14 & 12 & -.16 \\
\hline 15 & 13 & -.20 \\
\hline 16 & 14 & -.25 \\
\hline 17 & 15 & -.45 \\
\hline 18 & 12 & -.16 \\
\hline 19 & 12 & -.16 \\
\hline 20 & 10 & -.20 \\
\hline 21 & 13 & -.22 \\
\hline 22 & 9 & -.10 \\
\hline 23 & 7 & -.05 \\
\hline 24 & 5 & -.05 \\
\hline
\end{tabular}

Table 3-1: Hourly Day Ahead RTP with Self Elasticity Coefficients

\subsubsection{Long Range or Optimizing Consumers}

This category comprises optimizing consumers who can shift their consumption over a wide range of time following changes in prices and hence, their PEMs will have more non-zero entries corresponding to self and cross elasticity coefficients. The developed $24 \mathrm{X} 24$ matrix for LR consumer is shown in the table 3-2. It should be noted that self elasticity coefficients are not constant. The same values of self elasticity coefficients will be used for other categories of consumers as well. Some observations to be made from the PEM of LR consumer are as follows. 
- With respect to the prices in table 3-1, all elements below diagonal are zero for low pricing periods (column 1-7) because no consumer would shift his load from a low price period to a high price period.

- Some weight in the value of cross elasticity is given for shift within short range of time. For example, a shift from $t_{20}$ to $t_{24}$ is more prevalent than a shift from $t_{12}$ to $t_{24}$ despite the fact that price at $t_{12}$ and $t_{20}$ are the same $(10$ cents/KWH). Thus, a fading effect has been incorporated to take this rational logic into account. To explain this in detail, consider the case where cross elasticity for load shift from $t_{13}$ to $t_{6}$ is given a higher value of 0.04 , while shift from $t_{13}$ to $t_{1}$ has a lower value of 0.02 (in spite of the fact that price at $t_{6}$ is the same as price at $t_{1}$ ). This is because shifting load to a nearer time slot is more convenient for any customer.

\subsubsection{Short Range Consumers}

These consumers do not optimize their load and are only concerned about the price at the current time instant. Hence, The PEM will only have diagonal elements implying that there is only the self elasticity component that has varying coefficients and the cross elasticity coefficients are all zeros implying no load redistribution by the consumer. The 24X24 PEM of SR consumers is shown in table 3-3.

\subsubsection{Real World Postponing Consumer}

This category comprises consumers whose perception depends on current and future prices only and their perception is for 5 hours into the future. The $24 * 24$ PEM of a RW Postponing consumer is shown in table 3-4.

The following observations can be made regarding the behavior of RW Postponing consumer.

- If we observe the cross elasticity values here, they would be higher compared to corresponding values in LR's PEM. The reason is, unlike LR consumers who optimize their load throughout the day, these consumers only shift their load over a short range of five hours into the future.

- These consumers will be more willing to shift their loads within the available time range.

\subsubsection{Real World Advancing Consumer}

These consumers have PEM similar to postponing consumers with the only difference of the non-zero elements on and above the diagonal implying consumer perception into current and past time periods (for a period of 5 hours into the past). The PEM of a RW Advancing consumer is shown in table 3-5. 
Some observations to be made from the PEM of RW advancing consumer are as follows.

- On a 24X24 PEM, it can be seen that load is shifted around the high price period of $\mathrm{t}_{10}$ to $\mathrm{t}_{21}$ about the diagonal.

- These consumers differ from LR consumers in the fact that their flexibility ranges over a period of only 5 hours.

\subsubsection{Real World- Mixed Consumers}

These consumers are a mix of postponing and advancing consumers. Their perception goes 5 hours into the past and future which means they will have non-zero elements both above and below the diagonal. These consumers differ from LR consumers in the fact that their flexibility ranges over a period of 5 hours only whereas, LR consumers are flexible throughout the 24 hour period. The is shown in table 3-6. 


\begin{tabular}{|c|c|c|c|c|c|c|c|c|c|c|c|c|c|c|c|c|c|c|c|c|c|c|c|c|}
\hline & 1 & 2 & 3 & 4 & 5 & 6 & 7 & 8 & 9 & 10 & 11 & 12 & 13 & 14 & 15 & 16 & 17 & 18 & 19 & 20 & 21 & 22 & 23 & 24 \\
\hline 1 & -.01 & 0 & 0 & 0 & 0 & 0 & 0 & 0 & .01 & .02 & .02 & .02 & .02 & .02 & .02 & .02 & .02 & .015 & .01 & .01 & .01 & .01 & .01 & .01 \\
\hline 2 & 0 & -.01 & 0 & 0 & 0 & 0 & 0 & 0 & .01 & .02 & .02 & .02 & .02 & .02 & .02 & .02 & .02 & .015 & .01 & .01 & .01 & .01 & $\begin{array}{l}.01 \\
\end{array}$ & .01 \\
\hline 3 & 0 & 0 & -.01 & 0 & 0 & 0 & 0 & 0 & .01 & .02 & .02 & .02 & .02 & .02 & .02 & .02 & .02 & .015 & .015 & .01 & .01 & .01 & .01 & .01 \\
\hline 4 & 0 & 0 & 0 & -.01 & 0 & 0 & 0 & 0 & .01 & .02 & .02 & .02 & .02 & .02 & .02 & .02 & .04 & .015 & .015 & .02 & .015 & .015 & .015 & .01 \\
\hline 5 & 0 & 0 & 0 & 0 & -.01 & 0 & 0 & .015 & .035 & .04 & .04 & .04 & .04 & .04 & $\begin{array}{l}.04 \\
\end{array}$ & $\begin{array}{l}.04 \\
\end{array}$ & .042 & .03 & .03 & .022 & .015 & .02 & .015 & .015 \\
\hline 6 & 0 & 0 & 0 & 0 & 0 & -.01 & .01 & .015 & .035 & .04 & .04 & .04 & .04 & .04 & $\begin{array}{l}.04 \\
\end{array}$ & .043 & .045 & .03 & .03 & .026 & .02 & .023 & .02 & .017 \\
\hline 7 & 0 & 0 & 0 & 0 & 0 & 0 & -.01 & .015 & .02 & .027 & .03 & .03 & .03 & .04 & .033 & .035 & .038 & .026 & .026 & .022 & .025 & .019 & .015 & .012 \\
\hline 8 & 0 & 0 & 0 & 0 & 0 & 0 & & -.02 & .015 & .025 & .027 & .027 & .029 & .029 & .032 & .035 & .038 & .024 & .024 & .02 & .024 & .017 & .013 & .01 \\
\hline 9 & 0 & 0 & 0 & 0 & 0 & 0 & 0 & 0 & -.02 & .017 & .025 & .025 & .027 & .028 & .03 & .033 & .035 & .023 & .012 & .012 & .02 & $\begin{array}{l}.011 \\
\end{array}$ & .01 & 0 \\
\hline 10 & 0 & 0 & 0 & 0 & 0 & 0 & 0 & 0 & 0 & -.02 & .017 & .017 & .018 & .019 & .02 & .023 & .026 & .016 & .016 & .013 & .017 & .01 & 0 & 0 \\
\hline 11 & 0 & 0 & 0 & 0 & 0 & 0 & 0 & 0 & 0 & 0 & -.03 & 0 & .015 & .016 & .017 & .018 & .02 & .015 & .015 & 0 & .014 & 0 & 0 & 0 \\
\hline 12 & 0 & 0 & 0 & 0 & 0 & 0 & 0 & 0 & 0 & 0 & 0 & -.03 & .015 & .016 & .017 & .018 & .02 & .015 & .015 & 0 & .014 & 0 & 0 & 0 \\
\hline 13 & 0 & 0 & 0 & 0 & 0 & 0 & 0 & 0 & 0 & 0 & 0 & 0 & -.04 & .015 & .016 & .017 & .019 & .01 & .01 & 0 & .012 & 0 & 0 & 0 \\
\hline 14 & 0 & 0 & 0 & 0 & 0 & 0 & 0 & 0 & 0 & 0 & 0 & 0 & 0 & -.16 & .015 & .016 & .018 & 0 & 0 & 0 & .01 & 0 & 0 & 0 \\
\hline 15 & 0 & 0 & 0 & 0 & 0 & 0 & 0 & 0 & 0 & 0 & 0 & 0 & 0 & 0 & -.2 & $\begin{array}{l}.015 \\
\end{array}$ & .017 & 0 & 0 & 0 & 0 & 0 & 0 & 0 \\
\hline 16 & 0 & 0 & 0 & 0 & 0 & 0 & 0 & 0 & 0 & 0 & 0 & 0 & 0 & 0 & 0 & -.25 & .016 & 0 & 0 & 0 & 0 & 0 & 0 & 0 \\
\hline 17 & 0 & 0 & 0 & 0 & 0 & 0 & 0 & 0 & 0 & 0 & 0 & 0 & 0 & 0 & 0 & 0 & -.45 & 0 & 0 & 0 & 0 & 0 & 0 & 0 \\
\hline 18 & 0 & 0 & 0 & 0 & 0 & 0 & 0 & 0 & 0 & 0 & 0 & 0 & 0 & 0 & .01 & 0 & .021 & -.16 & 0 & 0 & .01 & 0 & 0 & 0 \\
\hline 19 & 0 & 0 & 0 & 0 & 0 & 0 & 0 & 0 & 0 & 0 & 0 & 0 & 0 & 0 & .01 & 0 & .021 & 0 & -.16 & 0 & .01 & 0 & 0 & 0 \\
\hline 20 & 0 & 0 & 0 & 0 & 0 & 0 & 0 & 0 & 0 & 0 & 0 & 0 & .01 & $\begin{array}{l}.014 \\
\end{array}$ & .016 & $\begin{array}{l}.015 \\
\end{array}$ & .025 & .016 & .016 & -.2 & .025 & 0 & 0 & 0 \\
\hline 21 & 0 & 0 & 0 & 0 & 0 & 0 & 0 & 0 & 0 & 0 & 0 & 0 & 0 & 0 & 0 & 0 & .018 & 0 & 0 & 0 & -.22 & 0 & 0 & 0 \\
\hline 22 & 0 & 0 & 0 & 0 & 0 & 0 & 0 & 0 & 0 & 0 & 0 & 0 & .011 & .015 & .016 & .018 & .027 & .018 & .018 & .013 & .027 & -.1 & 0 & 0 \\
\hline 23 & 0 & 0 & 0 & 0 & 0 & 0 & 0 & 0 & 0 & $\begin{array}{l}.01 \\
\end{array}$ & .01 & .01 & $\begin{array}{l}.015 \\
\end{array}$ & $\begin{array}{l}.02 \\
\end{array}$ & .022 & .034 & .035 & .02 & .02 & .016 & $\begin{array}{l}.029 \\
\end{array}$ & 016 & $\begin{array}{l}-.05 \\
\end{array}$ & 0 \\
\hline 24 & 0 & 0 & 0 & 0 & 0 & 0 & 0 & 0 & .01 & .02 & .02 & .02 & .02 & .025 & $\begin{array}{l}.03 \\
\end{array}$ & .035 & $\begin{array}{l}.039 \\
\end{array}$ & .03 & .03 & .028 & $\begin{array}{l}.032 \\
\end{array}$ & .03 & .018 & -.05 \\
\hline
\end{tabular}

Table 3-2: PEM of LR Consumer 


\begin{tabular}{|c|c|c|c|c|c|c|c|c|c|c|c|c|c|c|c|c|c|c|c|c|c|c|c|c|}
\hline & 1 & 2 & 3 & 4 & 5 & 6 & 7 & 8 & 9 & 10 & 11 & 12 & 13 & 14 & 15 & 16 & 17 & 18 & 19 & 20 & 21 & 22 & 23 & 24 \\
\hline 1 & -.01 & 0 & 0 & 0 & 0 & 0 & 0 & 0 & 0 & 0 & 0 & 0 & 0 & 0 & 0 & 0 & 0 & 0 & 0 & 0 & 0 & 0 & 0 & 0 \\
\hline 2 & 0 & -.01 & 0 & 0 & 0 & 0 & 0 & 0 & 0 & 0 & 0 & 0 & 0 & 0 & 0 & 0 & 0 & 0 & 0 & 0 & 0 & 0 & 0 & 0 \\
\hline 3 & 0 & 0 & -.01 & 0 & 0 & 0 & 0 & 0 & 0 & 0 & 0 & 0 & 0 & 0 & 0 & 0 & 0 & 0 & 0 & 0 & 0 & 0 & 0 & 0 \\
\hline 4 & 0 & 0 & 0 & -.01 & 0 & 0 & 0 & 0 & 0 & 0 & 0 & 0 & 0 & 0 & 0 & 0 & 0 & 0 & 0 & 0 & 0 & 0 & 0 & 0 \\
\hline 5 & 0 & 0 & 0 & 0 & -.01 & 0 & 0 & 0 & 0 & 0 & 0 & 0 & 0 & 0 & 0 & 0 & 0 & 0 & 0 & 0 & 0 & 0 & 0 & 0 \\
\hline 6 & 0 & 0 & 0 & 0 & 0 & -.01 & 0 & 0 & 0 & 0 & 0 & 0 & 0 & 0 & 0 & 0 & 0 & 0 & 0 & 0 & 0 & 0 & 0 & 0 \\
\hline 7 & 0 & 0 & 0 & 0 & 0 & 0 & -.01 & 0 & 0 & 0 & 0 & 0 & 0 & 0 & 0 & 0 & 0 & 0 & 0 & 0 & 0 & 0 & 0 & 0 \\
\hline 8 & 0 & 0 & 0 & 0 & 0 & 0 & 0 & -.02 & 0 & 0 & 0 & 0 & 0 & 0 & 0 & 0 & 0 & 0 & 0 & 0 & 0 & 0 & 0 & 0 \\
\hline 9 & 0 & 0 & 0 & 0 & 0 & 0 & 0 & 0 & -.02 & 0 & 0 & 0 & 0 & 0 & 0 & 0 & 0 & 0 & 0 & 0 & 0 & 0 & 0 & 0 \\
\hline 10 & 0 & 0 & 0 & 0 & 0 & 0 & 0 & 0 & 0 & -.02 & 0 & 0 & 0 & 0 & 0 & 0 & 0 & 0 & 0 & 0 & 0 & 0 & 0 & 0 \\
\hline 11 & 0 & 0 & 0 & 0 & 0 & 0 & 0 & 0 & 0 & 0 & -.03 & 0 & 0 & 0 & 0 & 0 & 0 & 0 & 0 & 0 & 0 & 0 & 0 & 0 \\
\hline 12 & 0 & 0 & 0 & 0 & 0 & 0 & 0 & 0 & 0 & 0 & 0 & -.03 & 0 & 0 & 0 & 0 & 0 & 0 & 0 & 0 & 0 & 0 & 0 & 0 \\
\hline 13 & 0 & 0 & 0 & 0 & 0 & 0 & 0 & 0 & 0 & 0 & 0 & 0 & -.04 & 0 & 0 & 0 & 0 & 0 & 0 & 0 & 0 & 0 & 0 & 0 \\
\hline 14 & 0 & 0 & 0 & 0 & 0 & 0 & 0 & 0 & 0 & 0 & 0 & 0 & 0 & -.16 & 0 & 0 & 0 & 0 & 0 & 0 & 0 & 0 & 0 & 0 \\
\hline 15 & 0 & 0 & 0 & 0 & 0 & 0 & 0 & 0 & 0 & 0 & 0 & 0 & 0 & 0 & -.2 & 0 & 0 & 0 & 0 & 0 & 0 & 0 & 0 & 0 \\
\hline 16 & 0 & 0 & 0 & 0 & 0 & 0 & 0 & 0 & 0 & 0 & 0 & 0 & 0 & 0 & 0 & -.25 & 0 & 0 & 0 & 0 & 0 & 0 & 0 & 0 \\
\hline 17 & 0 & 0 & 0 & 0 & 0 & 0 & 0 & 0 & 0 & 0 & 0 & 0 & 0 & 0 & 0 & 0 & -.45 & 0 & 0 & 0 & 0 & 0 & 0 & 0 \\
\hline 18 & 0 & 0 & 0 & 0 & 0 & 0 & 0 & 0 & 0 & 0 & 0 & 0 & 0 & 0 & 0 & 0 & 0 & -.16 & 0 & 0 & 0 & 0 & 0 & 0 \\
\hline 19 & 0 & 0 & 0 & 0 & 0 & 0 & 0 & 0 & 0 & 0 & 0 & 0 & 0 & 0 & 0 & 0 & 0 & 0 & -.16 & 0 & 0 & 0 & 0 & 0 \\
\hline 20 & 0 & 0 & 0 & 0 & 0 & 0 & 0 & 0 & 0 & 0 & 0 & 0 & 0 & 0 & 0 & 0 & 0 & 0 & 0 & -.2 & 0 & 0 & 0 & 0 \\
\hline 21 & 0 & 0 & 0 & 0 & 0 & 0 & 0 & 0 & 0 & 0 & 0 & 0 & 0 & 0 & 0 & 0 & 0 & 0 & 0 & 0 & -.22 & 0 & 0 & 0 \\
\hline 22 & 0 & 0 & 0 & 0 & 0 & 0 & 0 & 0 & 0 & 0 & 0 & 0 & 0 & 0 & 0 & 0 & 0 & 0 & 0 & 0 & 0 & -.1 & 0 & 0 \\
\hline 23 & 0 & 0 & 0 & 0 & 0 & 0 & 0 & 0 & 0 & 0 & 0 & 0 & 0 & 0 & 0 & 0 & 0 & 0 & 0 & 0 & 0 & 0 & -.05 & 0 \\
\hline 24 & 0 & 0 & 0 & 0 & 0 & 0 & 0 & 0 & 0 & 0 & 0 & 0 & 0 & 0 & 0 & 0 & 0 & 0 & 0 & 0 & 0 & 0 & 0 & -.05 \\
\hline
\end{tabular}

Table 3-3: PEM of SR Consumer 


\begin{tabular}{|c|c|c|c|c|c|c|c|c|c|c|c|c|c|c|c|c|c|c|c|c|c|c|c|c|}
\hline & 1 & 2 & 3 & 4 & 5 & 6 & 7 & 8 & 9 & 10 & 11 & 12 & 13 & 14 & 15 & 16 & 17 & 18 & 19 & 20 & 21 & 22 & 23 & 24 \\
\hline 1 & -.01 & 0 & 0 & 0 & 0 & 0 & 0 & 0 & 0 & 0 & 0 & 0 & 0 & 0 & 0 & 0 & 0 & 0 & 0 & 0 & 0 & 0 & 0 & 0 \\
\hline 2 & 0 & -.01 & 0 & 0 & 0 & 0 & 0 & 0 & 0 & 0 & 0 & 0 & 0 & 0 & 0 & 0 & 0 & 0 & 0 & 0 & 0 & 0 & 0 & 0 \\
\hline 3 & 0 & 0 & -.01 & 0 & 0 & 0 & 0 & 0 & 0 & 0 & 0 & 0 & 0 & 0 & 0 & 0 & 0 & 0 & 0 & 0 & 0 & 0 & 0 & 0 \\
\hline 4 & 0 & 0 & 0 & -.01 & 0 & 0 & 0 & 0 & 0 & 0 & 0 & 0 & 0 & 0 & 0 & 0 & 0 & 0 & 0 & 0 & 0 & 0 & 0 & 0 \\
\hline 5 & 0 & 0 & 0 & 0 & -.01 & 0 & 0 & 0 & 0 & 0 & 0 & 0 & 0 & 0 & 0 & 0 & 0 & 0 & 0 & 0 & 0 & 0 & 0 & 0 \\
\hline 6 & 0 & 0 & 0 & 0 & 0 & -.01 & 0 & 0 & 0 & 0 & 0 & 0 & 0 & 0 & 0 & 0 & 0 & 0 & 0 & 0 & 0 & 0 & 0 & 0 \\
\hline 7 & 0 & 0 & 0 & 0 & 0 & 0 & -.01 & 0 & 0 & 0 & 0 & 0 & 0 & 0 & 0 & 0 & 0 & 0 & 0 & 0 & 0 & 0 & 0 & 0 \\
\hline 8 & 0 & 0 & 0 & 0 & 0 & 0 & 0 & -.02 & 0 & 0 & 0 & 0 & 0 & 0 & 0 & 0 & 0 & 0 & 0 & 0 & 0 & 0 & 0 & 0 \\
\hline 9 & 0 & 0 & 0 & 0 & 0 & 0 & 0 & 0 & -.02 & 0 & 0 & 0 & 0 & 0 & 0 & 0 & 0 & 0 & 0 & 0 & 0 & 0 & 0 & 0 \\
\hline 10 & 0 & 0 & 0 & 0 & 0 & 0 & 0 & 0 & 0 & -.02 & 0 & 0 & 0 & 0 & 0 & 0 & 0 & 0 & 0 & 0 & 0 & 0 & 0 & 0 \\
\hline 11 & 0 & 0 & 0 & 0 & 0 & 0 & 0 & 0 & 0 & 0 & -.03 & 0 & 0 & 0 & 0 & 0 & 0 & 0 & 0 & 0 & 0 & 0 & 0 & 0 \\
\hline 12 & 0 & 0 & 0 & 0 & 0 & 0 & 0 & 0 & 0 & 0 & 0 & -.03 & 0 & 0 & 0 & 0 & 0 & 0 & 0 & 0 & 0 & 0 & 0 & 0 \\
\hline 13 & 0 & 0 & 0 & 0 & 0 & 0 & 0 & 0 & 0 & 0 & 0 & 0 & -.04 & 0 & 0 & 0 & 0 & 0 & 0 & 0 & 0 & 0 & 0 & 0 \\
\hline 14 & 0 & 0 & 0 & 0 & 0 & 0 & 0 & 0 & 0 & 0 & 0 & 0 & 0 & -.16 & 0 & 0 & 0 & 0 & 0 & 0 & 0 & 0 & 0 & 0 \\
\hline 15 & 0 & 0 & 0 & 0 & 0 & 0 & 0 & 0 & 0 & 0 & 0 & 0 & 0 & 0 & -.2 & 0 & 0 & 0 & 0 & 0 & 0 & 0 & 0 & 0 \\
\hline 16 & 0 & 0 & 0 & 0 & 0 & 0 & 0 & 0 & 0 & 0 & 0 & 0 & 0 & 0 & 0 & -.25 & 0 & 0 & 0 & 0 & 0 & 0 & 0 & 0 \\
\hline 17 & 0 & 0 & 0 & 0 & 0 & 0 & 0 & 0 & 0 & 0 & 0 & 0 & 0 & 0 & 0 & 0 & -.45 & 0 & 0 & 0 & 0 & 0 & 0 & 0 \\
\hline 18 & 0 & 0 & 0 & 0 & 0 & 0 & 0 & 0 & 0 & 0 & 0 & 0 & 0 & 0 & .02 & 0 & .025 & -.16 & 0 & 0 & 0 & 0 & 0 & 0 \\
\hline 19 & 0 & 0 & 0 & 0 & 0 & 0 & 0 & 0 & 0 & 0 & 0 & 0 & 0 & 0 & .02 & 0 & .025 & 0 & -.16 & 0 & 0 & 0 & 0 & 0 \\
\hline 20 & 0 & 0 & 0 & 0 & 0 & 0 & 0 & 0 & 0 & 0 & 0 & 0 & 0 & 0 & .025 & .02 & .03 & .02 & 0 & -.2 & 0 & 0 & 0 & 0 \\
\hline 21 & 0 & 0 & 0 & 0 & 0 & 0 & 0 & 0 & 0 & 0 & 0 & 0 & 0 & 0 & 0 & 0 & .02 & 0 & .019 & 0 & -.22 & 0 & 0 & 0 \\
\hline 22 & 0 & 0 & 0 & 0 & 0 & 0 & 0 & 0 & 0 & 0 & 0 & 0 & 0 & 0 & 0 & 0 & .032 & .022 & 0 & .016 & .03 & -.1 & 0 & 0 \\
\hline 23 & 0 & 0 & 0 & 0 & 0 & 0 & 0 & 0 & 0 & 0 & 0 & 0 & 0 & 0 & 0 & 0 & 0 & .025 & .022 & .019 & .032 & .019 & -.05 & 0 \\
\hline 24 & 0 & 0 & 0 & 0 & 0 & 0 & 0 & 0 & 0 & 0 & 0 & 0 & 0 & 0 & 0 & 0 & 0 & 0 & .025 & .035 & .035 & .035 & .018 & -.05 \\
\hline
\end{tabular}




\begin{tabular}{|c|c|c|c|c|c|c|c|c|c|c|c|c|c|c|c|c|c|c|c|c|c|c|c|c|}
\hline & 1 & 2 & 3 & 4 & 5 & 6 & 7 & 8 & 9 & 10 & 11 & 12 & 13 & 14 & 15 & 16 & 17 & 18 & 19 & 20 & 21 & 22 & 23 & 24 \\
\hline 1 & -.01 & 0 & 0 & 0 & 0 & 0 & 0 & 0 & 0 & 0 & 0 & 0 & 0 & 0 & 0 & 0 & 0 & 0 & 0 & 0 & 0 & 0 & 0 & 0 \\
\hline 2 & 0 & -.01 & 0 & 0 & 0 & 0 & 0 & 0 & 0 & 0 & 0 & 0 & 0 & 0 & 0 & 0 & 0 & 0 & 0 & 0 & 0 & 0 & 0 & 0 \\
\hline 3 & 0 & 0 & -.01 & 0 & 0 & 0 & 0 & 0 & 0 & 0 & 0 & 0 & 0 & 0 & 0 & 0 & 0 & 0 & 0 & 0 & 0 & 0 & 0 & 0 \\
\hline 4 & 0 & 0 & 0 & -.01 & 0 & 0 & 0 & 0 & .04 & 0 & 0 & 0 & 0 & 0 & 0 & 0 & 0 & 0 & 0 & 0 & 0 & 0 & 0 & 0 \\
\hline 5 & 0 & 0 & 0 & 0 & -.01 & 0 & .02 & .02 & .04 & .04 & 0 & 0 & 0 & 0 & 0 & 0 & 0 & 0 & 0 & 0 & 0 & 0 & 0 & 0 \\
\hline 6 & 0 & 0 & 0 & 0 & 0 & -.01 & .02 & .02 & .04 & .04 & .04 & 0 & 0 & 0 & 0 & 0 & 0 & 0 & 0 & 0 & 0 & 0 & 0 & 0 \\
\hline 7 & 0 & 0 & 0 & 0 & 0 & 0 & -.01 & .02 & .025 & .03 & .035 & .035 & 0 & 0 & 0 & 0 & 0 & 0 & 0 & 0 & 0 & 0 & 0 & 0 \\
\hline 8 & 0 & 0 & 0 & 0 & 0 & 0 & 0 & -.02 & .02 & .028 & .03 & .03 & .035 & 0 & 0 & 0 & 0 & 0 & 0 & 0 & 0 & 0 & 0 & 0 \\
\hline 9 & 0 & 0 & 0 & 0 & 0 & 0 & 0 & 0 & -.02 & .022 & .028 & .028 & .03 & .03 & 0 & 0 & 0 & 0 & 0 & 0 & 0 & 0 & 0 & 0 \\
\hline 10 & 0 & 0 & 0 & 0 & 0 & 0 & 0 & 0 & 0 & -.02 & .02 & .02 & .02 & .024 & .035 & 0 & 0 & 0 & 0 & 0 & 0 & 0 & 0 & 0 \\
\hline 11 & 0 & 0 & 0 & 0 & 0 & 0 & 0 & 0 & 0 & 0 & -.03 & 0 & .018 & .02 & .034 & .025 & 0 & 0 & 0 & 0 & 0 & 0 & 0 & 0 \\
\hline 12 & 0 & 0 & 0 & 0 & 0 & 0 & 0 & 0 & 0 & 0 & 0 & -.03 & .018 & .02 & .02 & .025 & .026 & 0 & 0 & 0 & 0 & 0 & 0 & 0 \\
\hline 13 & 0 & 0 & 0 & 0 & 0 & 0 & 0 & 0 & 0 & 0 & 0 & 0 & -.04 & .018 & .02 & .022 & .024 & .02 & 0 & 0 & 0 & 0 & 0 & 0 \\
\hline 14 & 0 & 0 & 0 & 0 & 0 & 0 & 0 & 0 & 0 & 0 & 0 & 0 & 0 & -.16 & .018 & .02 & .022 & 0 & 0 & 0 & 0 & 0 & 0 & 0 \\
\hline 15 & 0 & 0 & 0 & 0 & 0 & 0 & 0 & 0 & 0 & 0 & 0 & 0 & 0 & 0 & -0.2 & .018 & .02 & 0 & 0 & 0 & 0 & 0 & 0 & 0 \\
\hline 16 & 0 & 0 & 0 & 0 & 0 & 0 & 0 & 0 & 0 & 0 & 0 & 0 & 0 & 0 & 0 & -.25 & .019 & 0 & 0 & 0 & 0 & 0 & 0 & 0 \\
\hline 17 & 0 & 0 & 0 & 0 & 0 & 0 & 0 & 0 & 0 & 0 & 0 & 0 & 0 & 0 & 0 & 0 & -.45 & 0 & 0 & 0 & 0 & 0 & 0 & 0 \\
\hline 18 & 0 & 0 & 0 & 0 & 0 & 0 & 0 & 0 & 0 & 0 & 0 & 0 & 0 & 0 & 0 & 0 & 0 & -.16 & 0 & 0 & .02 & 0 & 0 & 0 \\
\hline 19 & 0 & 0 & 0 & 0 & 0 & 0 & 0 & 0 & 0 & 0 & 0 & 0 & 0 & 0 & 0 & 0 & 0 & 0 & -.16 & 0 & .02 & 0 & 0 & 0 \\
\hline 20 & 0 & 0 & 0 & 0 & 0 & 0 & 0 & 0 & 0 & 0 & 0 & 0 & 0 & 0 & 0 & 0 & 0 & 0 & 0 & -.2 & .03 & 0 & 0 & 0 \\
\hline 21 & 0 & 0 & 0 & 0 & 0 & 0 & 0 & 0 & 0 & 0 & 0 & 0 & 0 & 0 & 0 & 0 & 0 & 0 & 0 & 0 & -.22 & 0 & 0 & 0 \\
\hline 22 & 0 & 0 & 0 & 0 & 0 & 0 & 0 & 0 & 0 & 0 & 0 & 0 & 0 & 0 & 0 & 0 & 0 & 0 & 0 & 0 & 0 & -.1 & 0 & 0 \\
\hline 23 & 0 & 0 & 0 & 0 & 0 & 0 & 0 & 0 & 0 & 0 & 0 & 0 & 0 & 0 & 0 & 0 & 0 & 0 & 0 & 0 & 0 & 0 & -.05 & 0 \\
\hline 24 & 0 & 0 & 0 & 0 & 0 & 0 & 0 & 0 & 0 & 0 & 0 & 0 & 0 & 0 & 0 & 0 & 0 & 0 & 0 & 0 & 0 & 0 & 0 & -.05 \\
\hline
\end{tabular}




\begin{tabular}{|c|c|c|c|c|c|c|c|c|c|c|c|c|c|c|c|c|c|c|c|c|c|c|c|c|}
\hline & 1 & 2 & 3 & 4 & 5 & 6 & 7 & 8 & 9 & 10 & 11 & 12 & 13 & 14 & 15 & 16 & 17 & 18 & 19 & 20 & 21 & 22 & 23 & 24 \\
\hline 1 & -.01 & 0 & 0 & 0 & 0 & 0 & 0 & 0 & 0 & 0 & 0 & 0 & 0 & 0 & 0 & 0 & 0 & 0 & 0 & 0 & 0 & 0 & 0 & 0 \\
\hline 2 & 0 & -.01 & 0 & 0 & 0 & 0 & 0 & 0 & 0 & 0 & 0 & 0 & 0 & 0 & 0 & 0 & 0 & 0 & 0 & 0 & 0 & 0 & 0 & 0 \\
\hline 3 & 0 & 0 & -.01 & 0 & 0 & 0 & 0 & 0 & 0 & 0 & 0 & 0 & 0 & 0 & 0 & 0 & 0 & 0 & 0 & 0 & 0 & 0 & 0 & 0 \\
\hline 4 & 0 & 0 & 0 & -.01 & 0 & 0 & 0 & 0 & .04 & 0 & 0 & 0 & 0 & 0 & 0 & 0 & 0 & 0 & 0 & 0 & 0 & 0 & 0 & 0 \\
\hline 5 & 0 & 0 & 0 & 0 & -.01 & 0 & .02 & .02 & .04 & .04 & 0 & 0 & 0 & 0 & 0 & 0 & 0 & 0 & 0 & 0 & 0 & 0 & 0 & 0 \\
\hline 6 & 0 & 0 & 0 & 0 & 0 & -.01 & .02 & .02 & .04 & .04 & .04 & 0 & 0 & 0 & 0 & 0 & 0 & 0 & 0 & 0 & 0 & 0 & 0 & 0 \\
\hline 7 & 0 & 0 & 0 & 0 & 0 & 0 & -.01 & .02 & .025 & .03 & .035 & .035 & 0 & 0 & 0 & 0 & 0 & 0 & 0 & 0 & 0 & 0 & 0 & 0 \\
\hline 8 & 0 & 0 & 0 & 0 & 0 & 0 & 0 & -.02 & .02 & .028 & .03 & .03 & .035 & 0 & 0 & 0 & 0 & 0 & 0 & 0 & 0 & 0 & 0 & 0 \\
\hline 9 & 0 & 0 & 0 & 0 & 0 & 0 & 0 & 0 & -.02 & .022 & .028 & .028 & .03 & 0.03 & 0 & 0 & 0 & 0 & 0 & 0 & 0 & 0 & 0 & 0 \\
\hline 10 & 0 & 0 & 0 & 0 & 0 & 0 & 0 & 0 & 0 & -.02 & .02 & .02 & .02 & 0.024 & .035 & 0 & 0 & 0 & 0 & 0 & 0 & 0 & 0 & 0 \\
\hline 11 & 0 & 0 & 0 & 0 & 0 & 0 & 0 & 0 & 0 & 0 & -.03 & 0 & .018 & 0.02 & .034 & .025 & 0 & 0 & 0 & 0 & 0 & 0 & 0 & 0 \\
\hline 12 & 0 & 0 & 0 & 0 & 0 & 0 & 0 & 0 & 0 & 0 & 0 & -.03 & .018 & 0.02 & .02 & .025 & .026 & 0 & 0 & 0 & 0 & 0 & 0 & 0 \\
\hline 13 & 0 & 0 & 0 & 0 & 0 & 0 & 0 & 0 & 0 & 0 & 0 & 0 & -.04 & .018 & .02 & .022 & .024 & .02 & 0 & 0 & 0 & 0 & 0 & 0 \\
\hline 14 & 0 & 0 & 0 & 0 & 0 & 0 & 0 & 0 & 0 & 0 & 0 & 0 & 0 & -.16 & .018 & .02 & .022 & 0 & 0 & 0 & 0 & 0 & 0 & 0 \\
\hline 15 & 0 & 0 & 0 & 0 & 0 & 0 & 0 & 0 & 0 & 0 & 0 & 0 & 0 & 0 & -0.2 & .018 & .02 & 0 & 0 & 0 & 0 & 0 & 0 & 0 \\
\hline 16 & 0 & 0 & 0 & 0 & 0 & 0 & 0 & 0 & 0 & 0 & 0 & 0 & 0 & 0 & 0 & -.25 & .019 & 0 & 0 & 0 & 0 & 0 & 0 & 0 \\
\hline 17 & 0 & 0 & 0 & 0 & 0 & 0 & 0 & 0 & 0 & 0 & 0 & 0 & 0 & 0 & 0 & 0 & -.45 & 0 & 0 & 0 & 0 & 0 & 0 & 0 \\
\hline 18 & 0 & 0 & 0 & 0 & 0 & 0 & 0 & 0 & 0 & 0 & 0 & 0 & 0 & 0 & .02 & 0 & .025 & -.16 & 0 & 0 & .02 & 0 & 0 & 0 \\
\hline 19 & 0 & 0 & 0 & 0 & 0 & 0 & 0 & 0 & 0 & 0 & 0 & 0 & 0 & 0 & .02 & 0 & .025 & 0 & -.16 & 0 & .02 & 0 & 0 & 0 \\
\hline 20 & 0 & 0 & 0 & 0 & 0 & 0 & 0 & 0 & 0 & 0 & 0 & 0 & 0 & 0 & .025 & .02 & .03 & .02 & 0 & -.2 & .03 & 0 & 0 & 0 \\
\hline 21 & 0 & 0 & 0 & 0 & 0 & 0 & 0 & 0 & 0 & 0 & 0 & 0 & 0 & 0 & 0 & 0 & .02 & 0 & .019 & 0 & -.22 & 0 & 0 & 0 \\
\hline 22 & 0 & 0 & 0 & 0 & 0 & 0 & 0 & 0 & 0 & 0 & 0 & 0 & 0 & 0 & 0 & 0 & .032 & .022 & 0 & .016 & .03 & -.1 & 0 & 0 \\
\hline 23 & 0 & 0 & 0 & 0 & 0 & 0 & 0 & 0 & 0 & 0 & 0 & 0 & 0 & 0 & 0 & 0 & 0 & .025 & .022 & .019 & .032 & .019 & -.05 & 0 \\
\hline 24 & 0 & 0 & 0 & 0 & 0 & 0 & 0 & 0 & 0 & 0 & 0 & 0 & 0 & 0 & 0 & 0 & 0 & 0 & .025 & .035 & .035 & .035 & .018 & -.05 \\
\hline
\end{tabular}




\subsection{Microgrid Market Optimization}

The proposed microgrid market optimization has been formulated as a threefold linear programming problem for 24 hours that includes DR models, charge/discharge schedule for a BESS and other demand side resources to determine the optimum dispatch schedules for an islanded microgrid.

Microgrid controller determines the overall dispatch after the DR aggregator has determined the maximum levels of DR that it can bid into the microgrid market and also, the charge/discharge schedule of the BESS in the microgrid is determined.

\subsubsection{DR Aggregator}

For a successful bidding at the microgrid, DR Aggregators use the comprehensive DR models of their consumers which are now available in the form of PEM. For a given DR Aggregator, given its consumer's PEM, objective function is to maximize demand reduction. It can be formulated as:

Maximize

$$
\sum_{\tau=1}^{\tau=24}{ }_{\tau} \mathrm{e}_{\tau t} \quad t=1,24
$$

Subject to

$$
\rho_{\tau}^{\min } \leq{ }_{\tau} \leq \rho_{\tau}^{\max }
$$

Where,

$$
\tau=\text { Price bid for load reduction at time } \tau
$$

$e_{\tau t}=$ Elasticity coefficient corresponding to position $\tau t$ in the PEM of the DR Aggregator

\subsubsection{BESS}

The objective of BESS is maximizing revenue by optimal charging/discharging of power $\left(B P_{t}\right)$ according to the available utility real time price signal $\left(R T P_{t}\right)$ over the 24 hour period. This optimization is 
constrained by the charge/discharge power $\left(B P_{t}\right)$ and energy storage $\left(E_{t}\right)$ capabilities of the battery in addition to its SOC and DOD.

Maximize

$$
\sum_{t=1}^{t=24} B P_{t} X R T P_{t}
$$

Subject to

$$
\begin{aligned}
& 0 \leq B P_{t} \leq B P_{\max } \\
& 0 \leq E_{t} \leq E_{\max } \\
& S O C_{L L} \leq \sum_{t=1}^{t=24} B P_{t} *(1 \text { Hour }) \leq S O C_{U L} \quad \text { (During charging) } \\
& \sum_{t=1}^{t=24} B P_{t} *(1 \text { Hour }) \leq D O D \quad \text { (During discharging) }
\end{aligned}
$$

\subsubsection{DER, DR and BESS Dispatch}

The optimization problem aims at minimizing the operating cost of the microgrid and formulated as:

Minimize

$$
\sum_{t=1}^{t=24} \sum_{i=1}^{i=I} C_{i} G_{t i}+\sum_{t=1}^{t=24} \sum_{j=1}^{j=J} B_{j} D_{t j}
$$


Subject to

$$
\begin{gathered}
G_{i}^{\text {min }} \leq G_{t i} \leq G_{i}^{\text {max }} \quad t=1, T ; i=1, I \\
D_{j}^{\text {min }} \leq D_{t j} \leq D_{t j}^{\text {max }} \quad t=1, T ; j=1, J \\
\sum_{i=1}^{i=I} G_{t i}+\sum_{j=1}^{j=J} D_{t j}=P_{t} \quad t=1, T
\end{gathered}
$$

Where

$$
D_{t j}^{\max }=\sum_{\tau=1}^{T} e_{t \tau}^{j} D_{j 0} \quad t=1, T ; j=1, J
$$

$C_{i}=$ Price bid by Resource $i$ per KWH

$B_{j}=$ Price bid by DR Aggregator $j$ per KWH load reduced

$G_{t i}=\mathrm{KW}$ output of Resource $i$ at time $t$

$D_{t j}=\mathrm{KW}$ load reduced by DR Aggregator $j$ at time $t$

$I, J=$ Total number of bidding resources and total number of bidding DR Aggregators respectively.

$G_{i}^{\max }, G_{i}^{\min }=$ Maximum and minimum output of resource $i$

$D_{j}^{\text {min }}=$ Minimum load reduced by DR Aggregator $j$

$D_{t j}^{\max }=$ Maximum allowable $\mathrm{KW}$ reduction by DR Aggregator $j$ at time $t$

$e_{t \tau}^{j}=$ Elasticity coefficient corresponding to position $t \tau$ in the PEM of DR Aggregator $j$

$D_{j 0}=$ Base aggregated load at DR Aggregator $j$

$P_{t}=$ Gross demand (before DR) of the microgrid network at time $t$ 


\section{Chapter 4}

\section{SIMULATION TOOLS AND SOFTWARE}

This chapter briefly describes various software packages used in this thesis for modeling the electric distribution system, to study the effects of the DR models developed and formulating and solve the microgrid market optimization.

Several tools are available both commercially and as open source for modeling power distribution networks. Some of the software tools that are capable of solving 3-phase unbalanced distribution power flow are OpenDSS, Cymdist, Milsoft Windmil and RDAP. Each of these tools has their own advantages and limitations. After careful analysis and comparative studies, OpenDSS was chosen for distribution power flow for distribution systems and microgrid. The mathematical models developed for Demand Response have to be integrated with the distribution power flow in OpenDSS utilizing MATLAB.

The proposed microgrid market optimization has been formulated as a linear programming problem. Again, LP problems can be formulated in a variety of optimization engines namely MATLAB ${ }^{\text {es }}$ Linprog, IBM ILOG CPLEX Optimizer, General Algebraic Modeling System (GAMS), etc. Out of these available solvers, CPLEX was initially used to demonstrate the proposed microgrid optimization for a smaller test scenario. Matlab linear programming toolbox was further used for the 24 hour microgrid market optimization with demand side resources of DGs, BESS and DR. Matlab was more convenient to run the 24 hour microgrid optimization for the larger system since the DR models were already developed in it. The tools used in this research work are described with their capabilities and their specific significance in the subsequent sections.

\subsection{Open Distribution System Simulator (OpenDSS)}

OpenDSS (also called DSS) is a comprehensive electrical power system tool for electric utility distribution systems developed by Electric Power Research Institute (EPRI) [56]. OpenDSS is an open source tool for simulating distribution systems and performs various analyses namely power flow, harmonics and dynamics in frequency domain. OpenDSS provides a flexible research platform and scope for distribution planning and analysis, general multiphase AC circuit analysis, distributed generation interconnection analysis, annual load and generation profiles. Time series distribution analysis is supported by OpenDSS by which we can run annual load simulations along with daily/yearly power flow 
solution modes. The simulator can also be interfaced and driven from tools like Matlab through its command interface (COM interface). In addition, OpenDSS has developed test cases for all IEEE benchmark test feeders. The harmonic flow solution is a fundamental feature of OpenDSS and it provides a good platform for analyzing harmonic effects of wind turbines and inverter based PV on voltage regulators and capacitor switching. These capabilities of the tool have generated tremendous interest among utilities to perform various interconnection studies of their network in OpenDSS.

The general structure of OpenDSS is shown in figure 4-1 [57] and figure 4-2 shows a standalone user interface. As seen from figure 4-1, the simulation engine can be driven by three different ways.

- OpenDSS scripts - This is the simplest way to drive the simulation engine where the direct scripting codes are used to define the circuit and solve it.

- COM interface - External tools can also be used to drive the engine provided they are compatible with the COM interface.

- User Written DLL - Custom developed sophisticated models can be incorporated in the simulation engine by writing suitable DLL and linking with the engine.

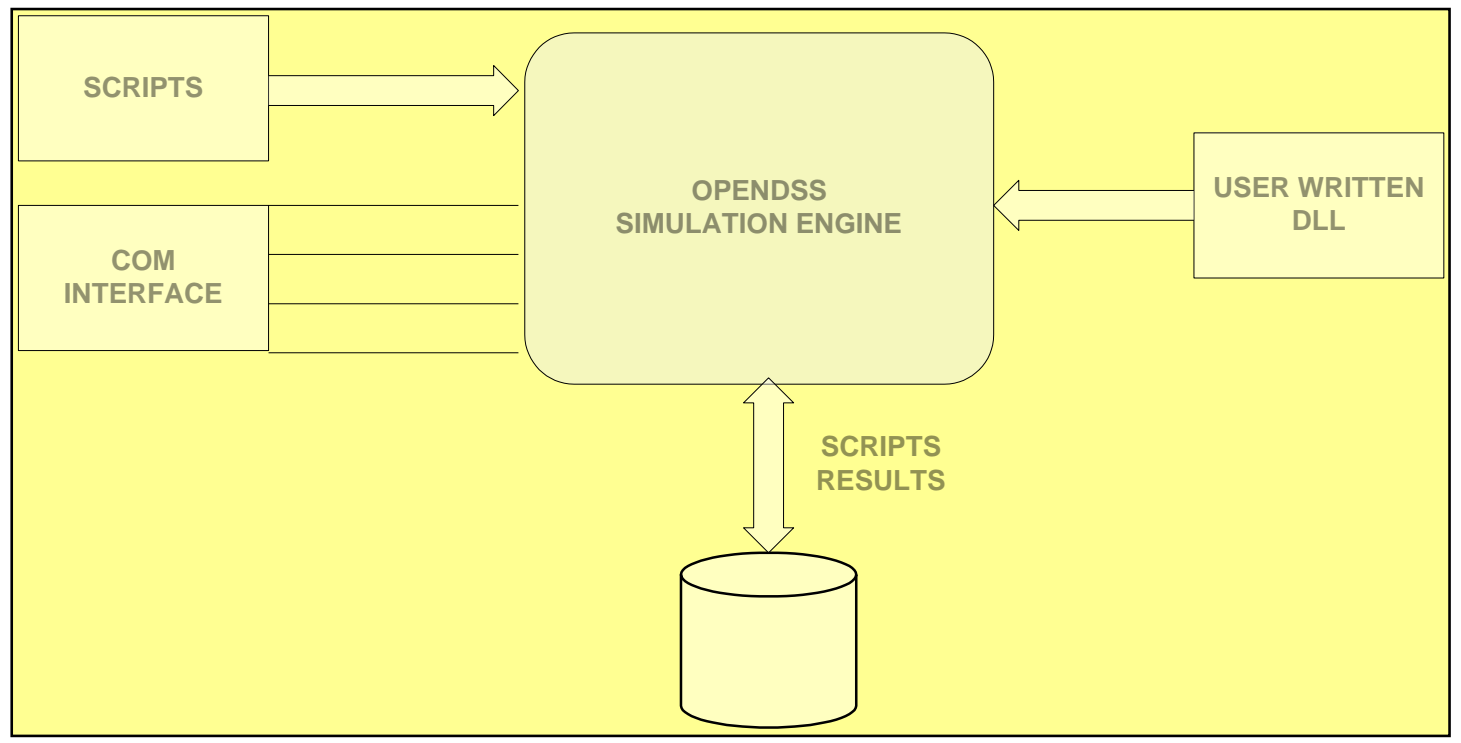

Figure 4-1: DSS Structure 


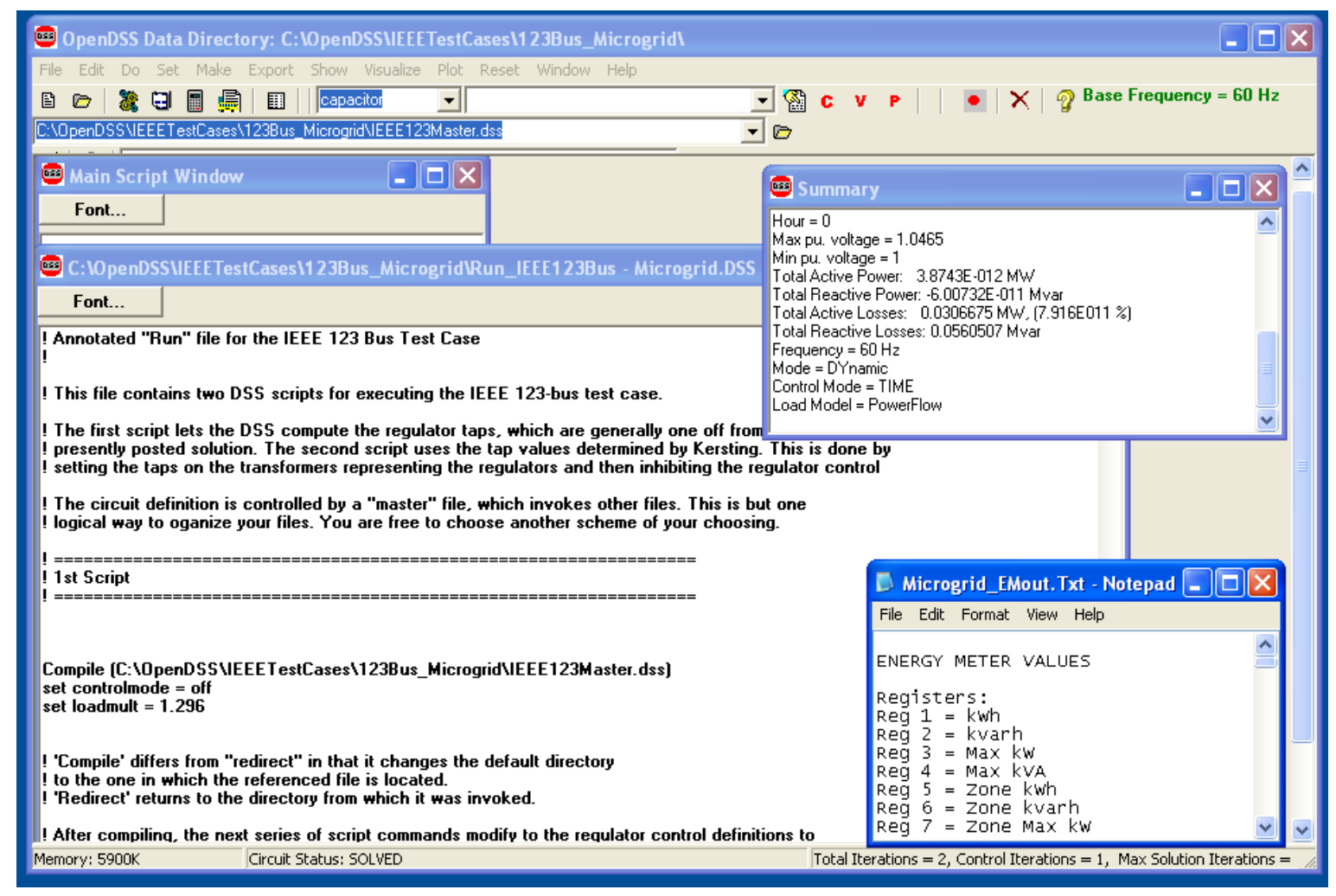

Figure 4-2: OpenDSS Standalone EXE User Interface

Some of the exclusive features of OpenDSS used in this work are listed below.

\subsubsection{Wide Range of Solution Modes}

A number of solution modes are offered by OpenDSS. The most important among these solution modes are shown in figure 4-3.

OpenDSS performs a basic distribution-style power flow in which the bulk power system is the dominant source of energy. It has the capability to solve both networked (meshed) systems as well as radial systems. The circuit model employed can be either a full multi-phase model or a positive sequence model. The snapshot power flow mode does one power flow solution at the present load level whereas the daily power flow mode does 24 hour solution following load shape defined as "Daily". For a daily power flow mode the default load shape provided in the engine can be used, or a user defined load shape can be used. Loadshapes can be imported from a comma separated value (CSV) file or can be directly written to the script file in the form of load multipliers. Similar to the daily mode, yearly power flow simulation can also be employed with loadshape defined as "Yearly". 


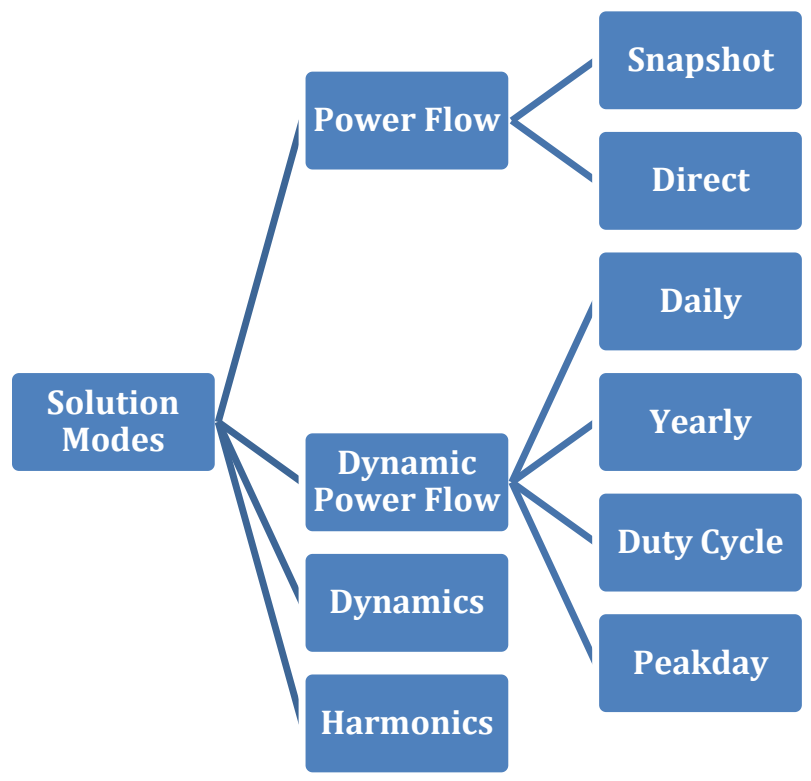

Figure 4-3: Solution Modes in OpenDSS

OpenDSS offers the "Dynamics" solution mode which is suitable for simple dynamics analysis for DG interconnection. This solution mode has been used in this thesis for simulating a microgrid test system to analyze the results of the proposed microgrid market optimization. The "Dynamics" solution mode must be preceded by a successful power flow. Changing to dynamics mode renders generators in the circuit as voltage sources behind specified reactances. This particular feature has been used in this work to model a moderately large radial distribution as an islanded microgrid with DGs.

\subsubsection{COM Interface}

The COM interface of OpenDSS is a very useful feature to execute custom solution modes and features externally and perform the functions of the simulator, including definition of the model data. This feature allows DSS to be implemented entirely independently of any database or fixed text file circuit definition. For example, it can be driven entirely from a MS Office tool through VBA, or from any other 3rd party analysis program that can handle COM such as MATLAB, Python, C\# and other languages. The COM interface also provides direct access to the text-based command interface by which an access to all the models available in the simulator can be obtained. Through the command interface, user-written programs can generate scripts to do several desired functions in sequence. Most of the results of the simulation engine can also be accessed through the COM interface.

In this thesis, the COM interface has been used to establish communication between MATLAB and OpenDSS. The mathematical formulations for DR model were developed in MATLAB and integrated with the distribution power flow in OpenDSS through COM interface. A snapshot of MATLAB 
commands used to register the COM server of OpenDSS and further to set the interface has been shown in figure 4-4.

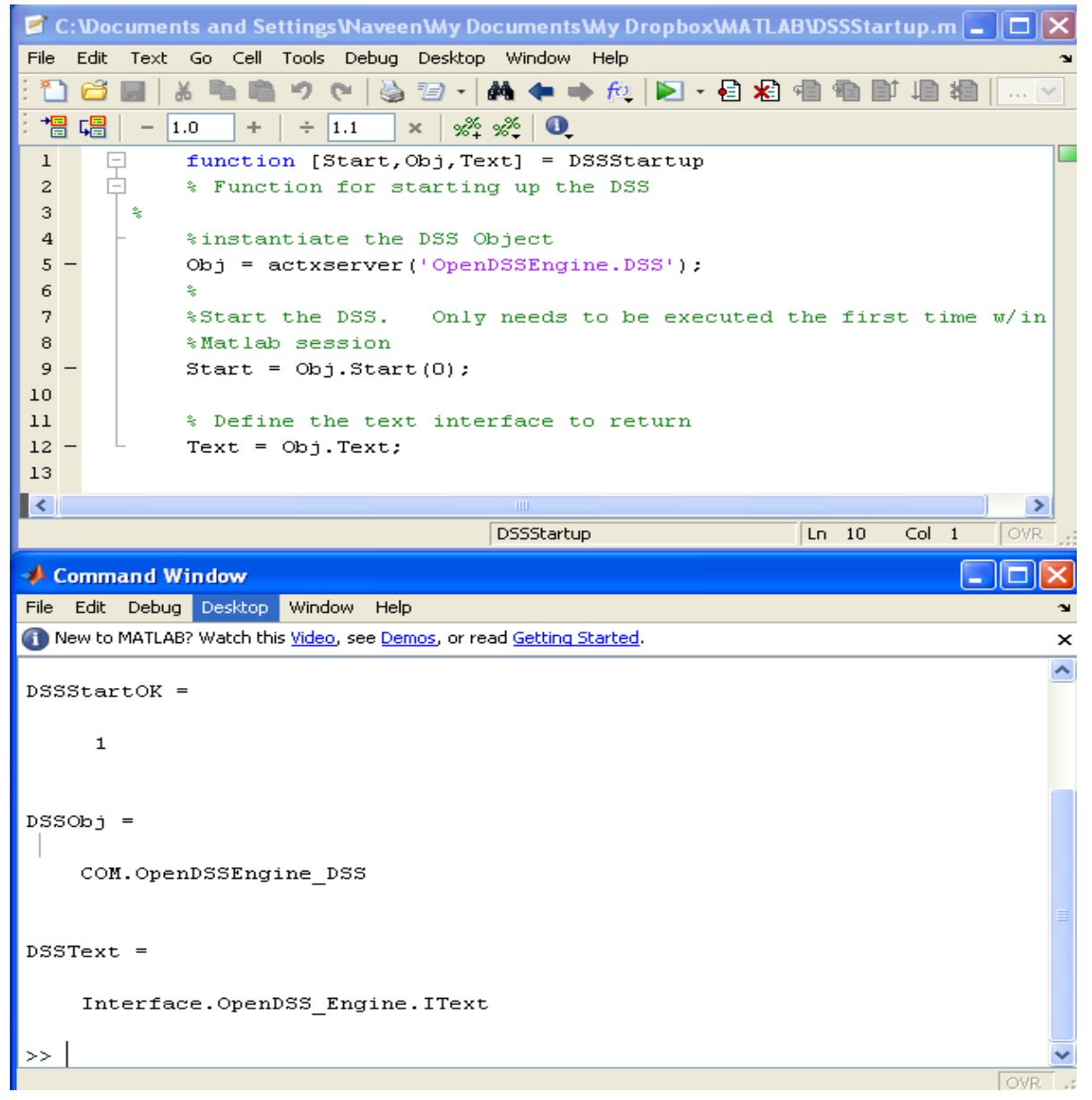

Figure 4-4: MATLAB-OpenDSS COM Interface

\subsection{MATLAB}

MATLAB ${ }^{\circledR}$, Matrix Laboratory, is a high level technical language and numerical computing environment for algorithm development, data visualization, data analysis, and numeric computation. Some of the key features of MATLAB are listed below [58].

- Developing environment for managing code, files, and data

- High level language for technical computing

- Tools for creating custom graphical user interfaces

- Functions for integrating MATLAB based methods to external applications, such as $\mathrm{C}, \mathrm{C}++$, FORTRAN, Java, and Microsoft Excel

- $\quad$ 2-D and 3-D graphics functions for visualizing data. 
The DR model developed in this thesis is primarily derived from the proposed price elasticity matrices. These 24X24 matrices that represent the DR patterns of consumers have been programmed in MATLAB since the operation was based on matrix and vectors.

Further, the developed DR models have been integrated with distribution power flow in OpenDSS by establishing a command interface between these two tools as explained in section 4.1.2. Commands from MATLAB are passed to OpenDSS directly as strings using the "text" object of the COM interface. Using this text interface, OpenDSS was driven from MATLAB as and when required. The snapshot in figure 4-5 shows the compilation of the test system in OpenDSS was compiled from MATLAB using text interface. It can also be seen that the main script file in OpenDSS can be edited using MATLAB commands.

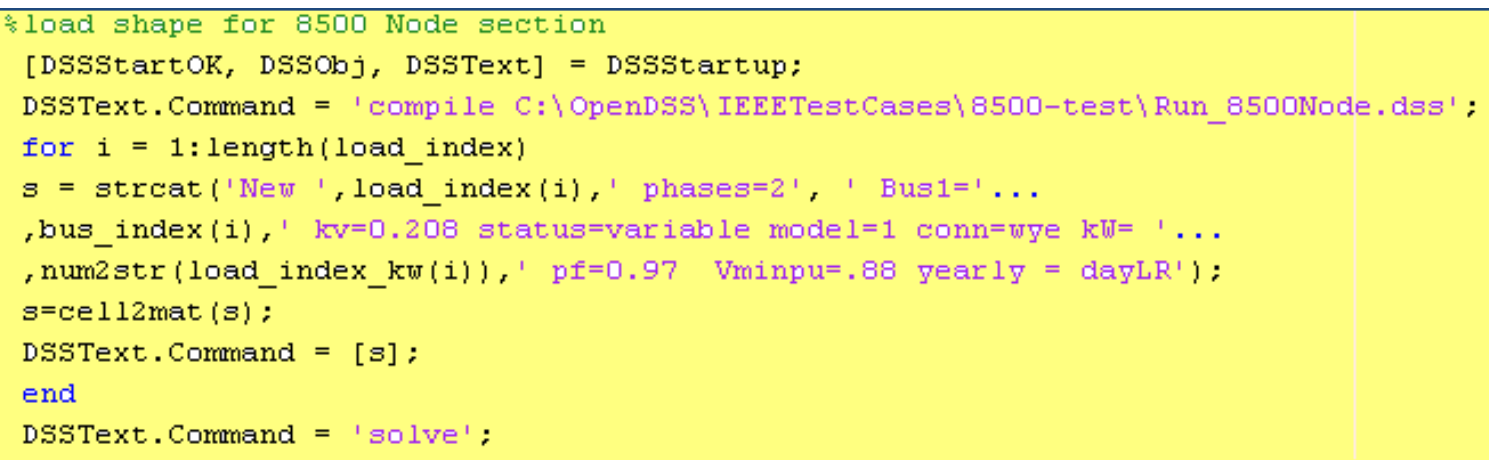

Figure 4-5: Snapshot showing MATLAB commands driving OpenDSS Engine using "text" object of COM Interface

In addition to these functions, MATLAB was extensively used to generate 2-D and surface plots of voltage analysis and loss analysis results of OpenDSS due to its superior plotting capabilities.

\subsubsection{MATLAB Optimization Toolbox}

The optimization toolbox of MATLAB provides algorithms to solve constrained and unconstrained continuous and discrete problems. The toolbox includes functions for linear programming, quadratic programming, binary integer programming, nonlinear optimization, nonlinear least squares, systems of nonlinear equations, and multiobjective optimization.

In this work, the linear programming toolbox with simplex algorithm was used to solve the LP optimization problem that has been formulated for the microgrid market using the simplex algorithm. The key advantage of MATLAB is that it is convenient to solve the 24 hour LP problem involving daily curves for loads, DGs and 24 hour charge/discharge schedule for BESS. 


\subsection{IBM ILOG CPLEX Optimizer}

CPLEX optimizer is a high-performance mathematical programming solver for linear programming, mixed integer programming, and quadratic programming [59]. CPLEX Optimizer's mathematical programming technology enables analytical decision support for improving efficiency, reducing costs, and increasing profitability. The application CPLEX ranges from simple linear programming to complex quadratic programming with millions of constraints and variables. A prominent feature of CPLEX is that it can be easily driven from the command prompt. Also CPLEX models can be easily integrated into any application written in Java, .NET or C++.

Owing to the simplicity of CPLEX, the microgrid optimization problem was preliminarily formulated and tested using the CPLEX simplex LP solver for a smaller microgrid system with limited number of DGs, and IPPs. Initially, the LP was formulated only for 3 time instants to analyze the efficiency of the optimization program. This algorithm was later scaled up for a 24 hour LP which was solved in MATLAB. A snapshot of a simple LP formulation along with its execution in CPLEX is shown in figure $4-7$.

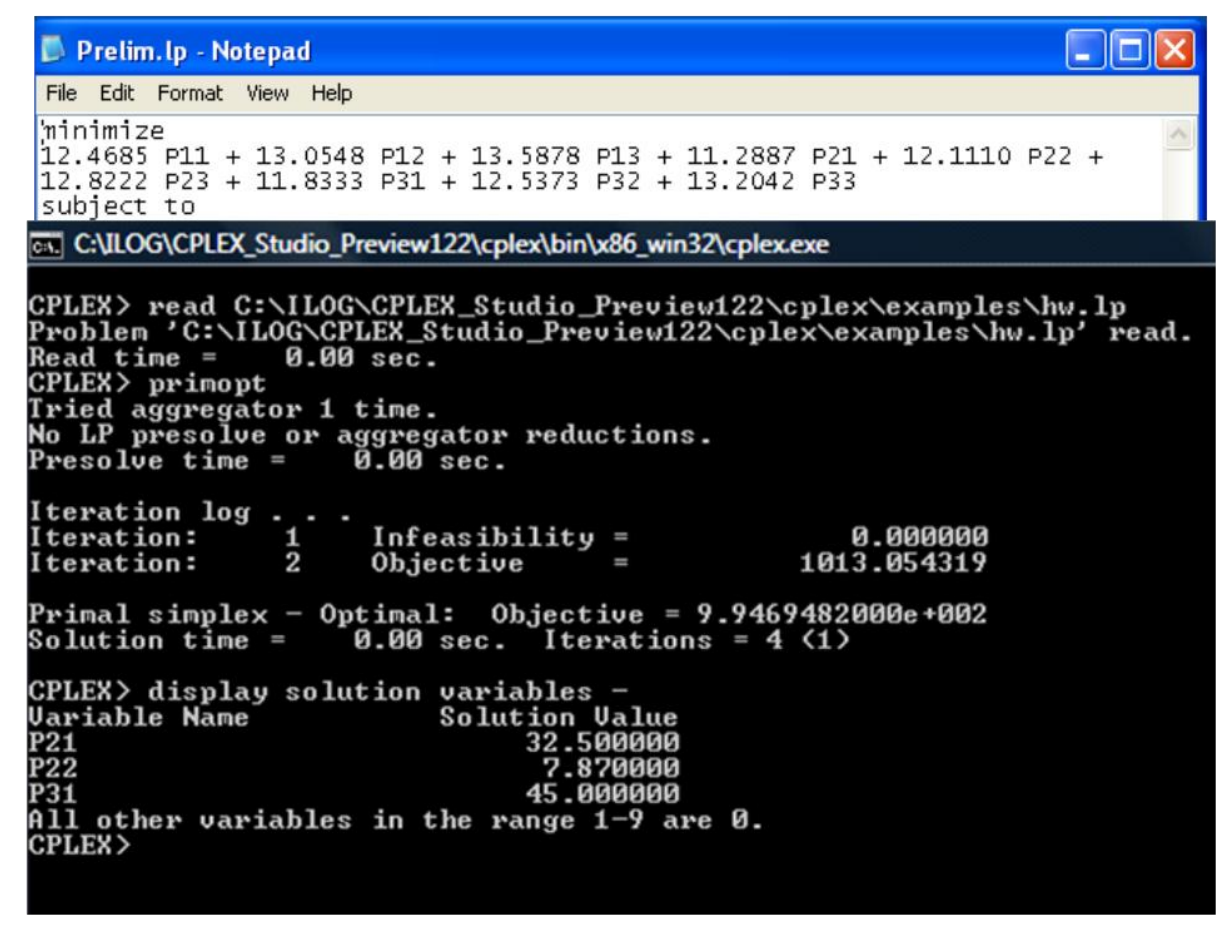

Figure 4-6: A snapshot of a simple LP formulation along with its execution in CPLEX 


\section{Chapter 5}

\section{SIMULATION AND RESULTS}

This chapter discusses all the results of this research by sequentially introducing the test systems used for the analysis and then the simulations performed using these test systems. Further, voltage and loss analysis results for the test systems are plotted. Inferences are drawn from these plots to support the proposed objectives and goals of this thesis work.

\subsection{IEEE 123 Node Radial Feeder}

The DR models developed were first integrated with IEEE 123 node test feeder [60] which is shown in figure 5-1. The IEEE 123 node test feeder operates at a nominal voltage of $4.16 \mathrm{kV}$ and has voltage drop problems. These voltage problems of the radial feeder have evoked interest to test the effect of the DR model on voltage profile of the system. Apart from the 8500 node radial feeder, this is the most comprehensive feeder and is characterized by overhead and underground line segments with various phasing, unbalanced loading with all combinations of load types (PQ, constant I, constant Z), a number of voltage regulators, shunt capacitor banks and switches.

The test system is divided into 4 different zones to group the loads according to their location as shown in table 5-1. This strategy has been employed so that the effect of DR can be systematically studied for each zone. It should be noted in the table that zones were determined by system mapping to resolve into 4 planes based on location. The load details column in the table lists all the loads, their phases and their parent node. 


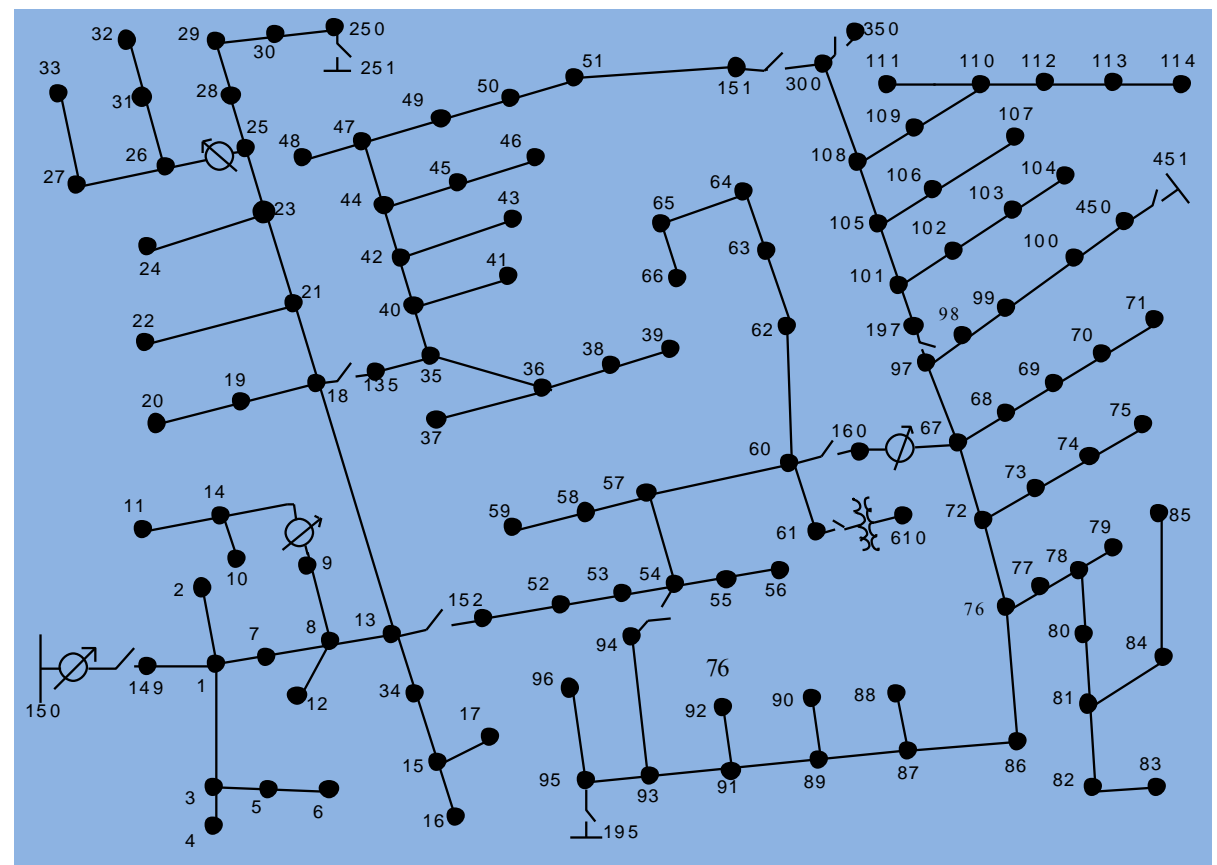

Figure 5-1: IEEE 123 node test feeder

\begin{tabular}{|c|c|c|c|}
\hline Zone & Nodes & $\begin{array}{c}\text { No. } \\
\text { of } \\
\text { Loads }\end{array}$ & Load details \\
\hline 1 & $\begin{array}{c}1,2,3,4,5,6,7,8,9,12,13 \\
15,16,17,34,36,37,38,39,52,53,54,55,57,58,59,91 \\
92,93,94,95,96,149,150,152\end{array}$ & 22 & $\begin{array}{l}\text { S1a,S2b,S4c,S5c,S6c, S7a,S9a,S12b,S16c, } \\
\text { S17c, S37a,S38b,S39b, S52a,S53a,S55a, } \\
\text { S58b,S59b,S92c,S94a,S95b,S96b, }\end{array}$ \\
\hline 2 & $\begin{array}{c}56,60,61,67,68,69,70,71,72,73,74,75,76,77,78,79,80 \\
81,82,83,84,85,86.87,88,89,90,160\end{array}$ & 23 & $\begin{array}{c}\text { S56b,S60a,S68a,S69a,S70a,S71a,S73c,S74c, } \\
\text { S75c,S76a,S76b,S76c,S77b,S79a,S80b,S82a, } \\
\text { S83c,S84c,S85c,S86b,S87b,S88a,S90b }\end{array}$ \\
\hline 3 & $\begin{array}{c}62,63,64,97,98,99,100,101,102,103,104,105 \\
106,107,108,109,110 \\
111,112,113,114,151,197,300,450\end{array}$ & 16 & $\begin{array}{c}\text { S62c,S63a,S64b,S98a,S99b,S100c,S102c, } \\
\text { S103c,S104c,S106b, S107b,S109a,S111a, } \\
\text { S112a,S113a,S114a }\end{array}$ \\
\hline 4 & $\begin{array}{c}10,11,14,18,19,20,21,22,23,24,25,26,27,28,29,30,31 \\
32,33,35,40,41,42,43,44,45,46,47,48,49,50,51,65,66 \\
135,250\end{array}$ & 30 & $\begin{array}{c}\mathrm{S} 10 \mathrm{a}, \mathrm{S} 11 \mathrm{a}, \mathrm{S} 19 \mathrm{a}, \mathrm{S} 20 \mathrm{a}, \\
\mathrm{S} 22 \mathrm{~b}, \mathrm{~S} 24 \mathrm{c}, \mathrm{S} 28 \mathrm{a}, \mathrm{S} 29 \mathrm{a}, \mathrm{S} 30 \mathrm{c}, \mathrm{S} 31 \mathrm{c}, \mathrm{S} 32 \mathrm{c}, \mathrm{S} 33 \mathrm{a}, \\
\mathrm{S} 34 \mathrm{c}, \mathrm{S} 35 \mathrm{a}, \mathrm{S} 41 \mathrm{c}, \mathrm{S} 42 \mathrm{a}, \mathrm{S} 43 \mathrm{~b}, \mathrm{~S} 45 \mathrm{a}, \mathrm{S} 46 \mathrm{a}, \mathrm{S} 47, \\
\text { S48,S49a,S49b,S49c,} \\
\text { S50c,S51a,S65a,S65b,S65c,S66c, }\end{array}$ \\
\hline
\end{tabular}

Table 5-1: Zone of 123 Node test Feeder 


\subsection{Extraction of DR Patterns from PEMs for IEEE 123 Node Feeder}

As the first step towards integration of DR into the distribution network, DR patterns for different consumer types were extracted from their respective PEMs as explained in section 3.1. To achieve this, fixed loads of the 123 node feeder were modeled as DR loads. Consider zone 3 that comprises three different load levels namely $20 \mathrm{KW}, 40 \mathrm{KW}$ and $75 \mathrm{KW}$. Utilizing the developed consumer models and price data as shown in table 3-1, the level of demand reduction is computed for each time instant according to equation 3.4 (With respect to equation 3.4, the 24 hour RTP curve of table 3-1 represents the variable tariff, $\mathrm{p}^{\mathrm{ee}}$. A fixed standard tariff has been used as the base price,, $\mathrm{p}_{0}{ }^{\mathrm{ee}}$. For a given consumer with a typical PEM, for any given value of base load, , $\mathrm{d}_{0}{ }^{e e}$ the corresponding demand curve, $\mathrm{d}^{\text {ee }}$ can be obtained).

Figures 5-2, 5-3 and 5-4 show the daily load curves for Long Range (Optimizing), Short Range and Real World Mixed Consumers on the zone 3 loads of the test feeder. Figure 5-2 shows that for a LR consumer, demand during peak-pricing hours has been either curtailed or redistributed to some other time periods. For an LR consumer, the level of DR penetration is quite high, having maximum consumptions during low-price periods and very low consumption during high-price period (from 13 hours to 21 hours).

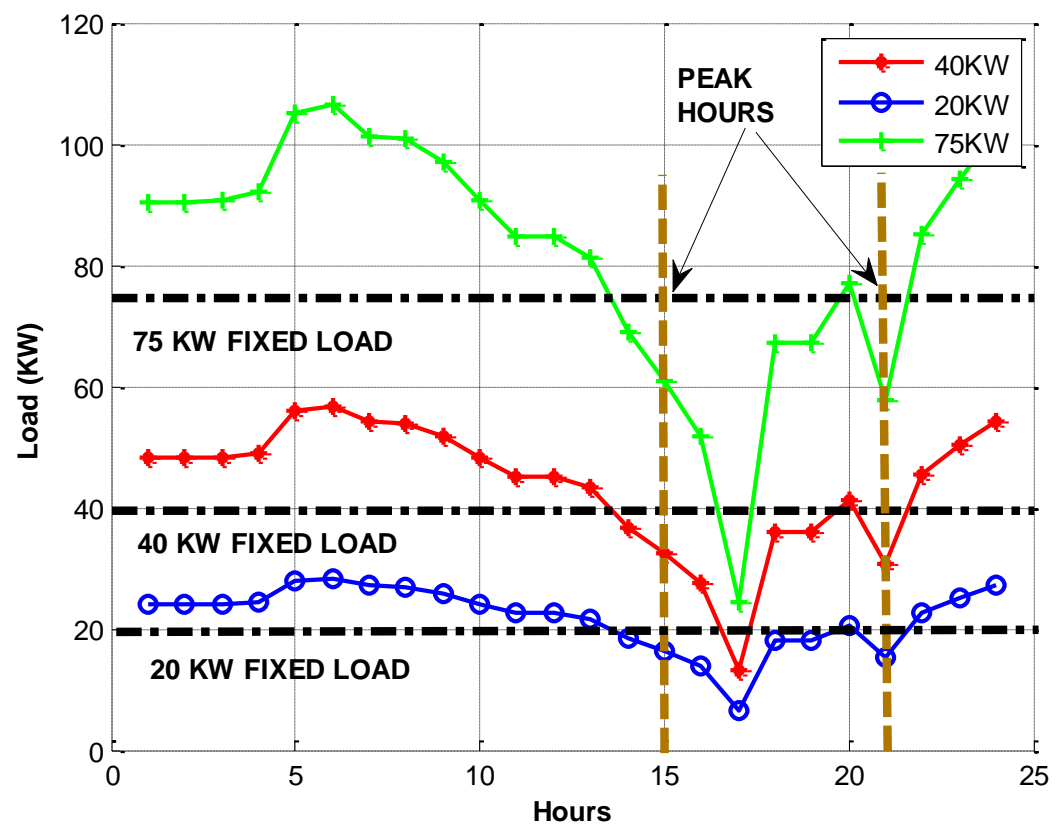

Figure 5-2: Daily demand curve of zone 3 loads for a LR consumer 
Figure 5-3 shows the demand curves for a SR consumer where demand curtailment is strictly restricted within the time frame of highest prices. Also, there is absolutely no redistribution of loads over time. The level of DR of a RW Mixed consumer lies in-between the DR pattern of a LR consumer and that of a SR consumer as seen in figure 5-4.

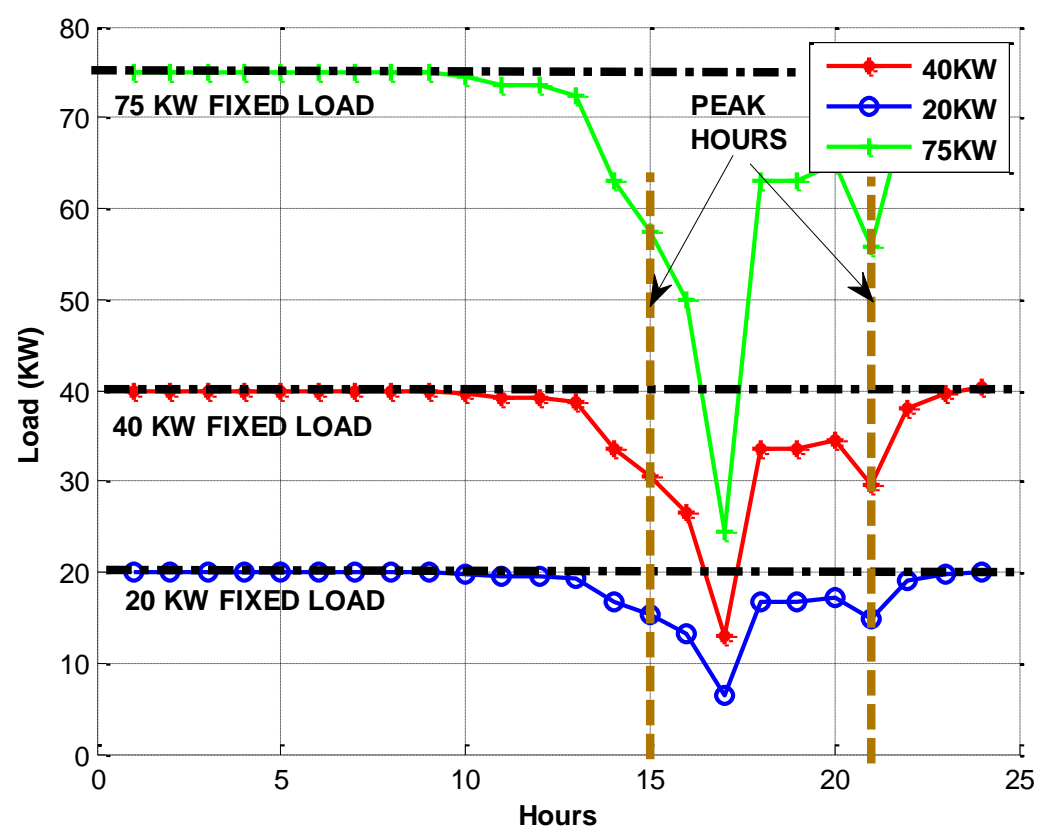

Figure 5-3: Daily demand curve of zone 3 loads for an SR consumer

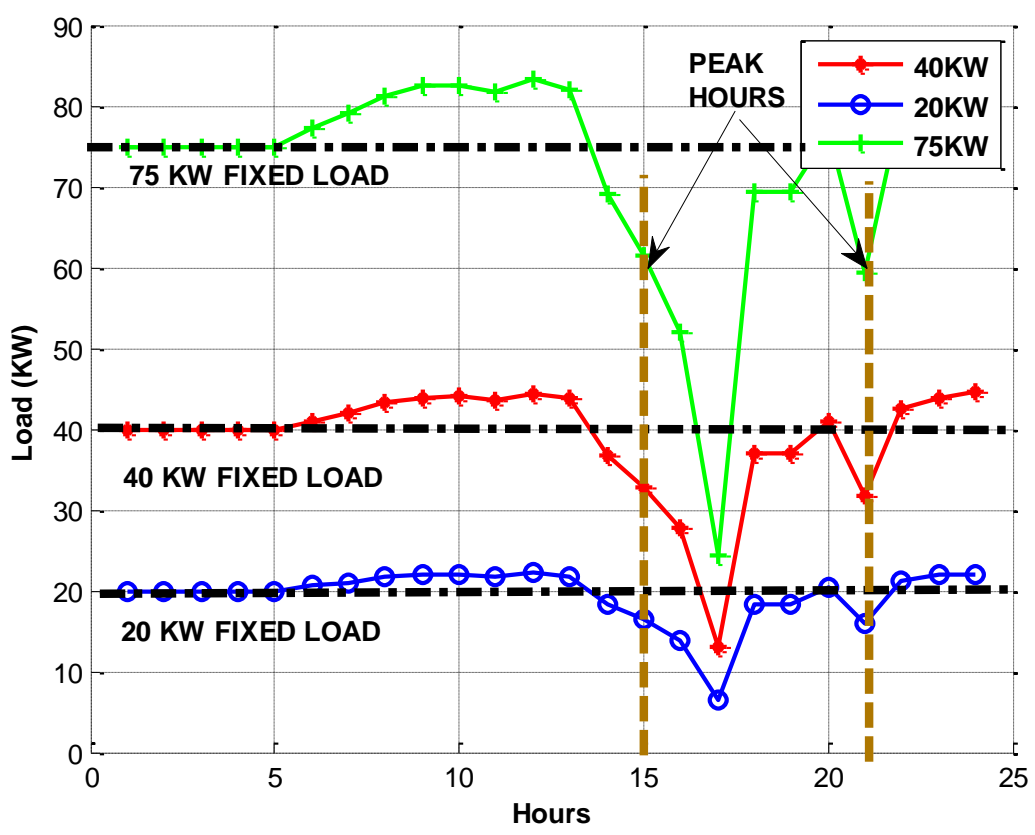

Figure 5-4: Daily demand curve of zone 3 loads for a RW Mixed consumer 
It can be noticed that the daily load curve of a LR consumer reflects a vigorous demand response pattern, since this type of consumer is most flexible with price change. The level of DR is lesser for RW Mixed consumer since the demand redistribution is confined only to five hours into the past and future (as seen in figure 5-4, five hours before and after the valley period). The DR level of SR consumer drops further down since this consumer does not redistribute loads and only reduces the load owing to a high price signal at a particular instant. It can also be noticed that the valley region (peak-pricing hours) is similar

for all the 3 types of consumers owing to the fact that self elasticity coefficients are assumed to be the same for different categories of consumers.

\subsection{Voltage Analysis of IEEE 123 Node Test Feeder}

Demand Response when applied at any part of a distribution network results in load change which in turn changes the power flows through the distribution transformers and the upstream areas of the distribution feeder. In case of load reduction due to DR, voltage drops across the distribution feeders reduce causing a boost in the voltage at the far end of the feeder. This effect is even more pronounced when DR is applied at the remote nodes of the feeder. However, there is a need to explore this DR-voltage relationship on a uniform basis throughout the day with optimum volt/var adjustments. Extensive deployment of AMI and availability of huge volumes of historical data and real time distribution operation models will provide better understanding on DR-voltage relationship in the long run.

The 3-phase unbalanced distribution power flow was obtained using OpenDSS for the 123 node test system. After a base case solution, loads were modeled over a period of 24 hours according to the daily load curves obtained in section 5.2. Loads were modeled both with non responsive and responsive load profiles for zone 3. Phase voltage profiles for nodes 62, 99,104 and 113 peak hours (12-20) are shown in figure 5-5 for loads without DR and loads with DR.

The plots show that system voltage is increased with DR allowing for further demand curtailment based on voltage reduction. It can be observed that DR during peak hours boosts the node voltages which would otherwise sag. 


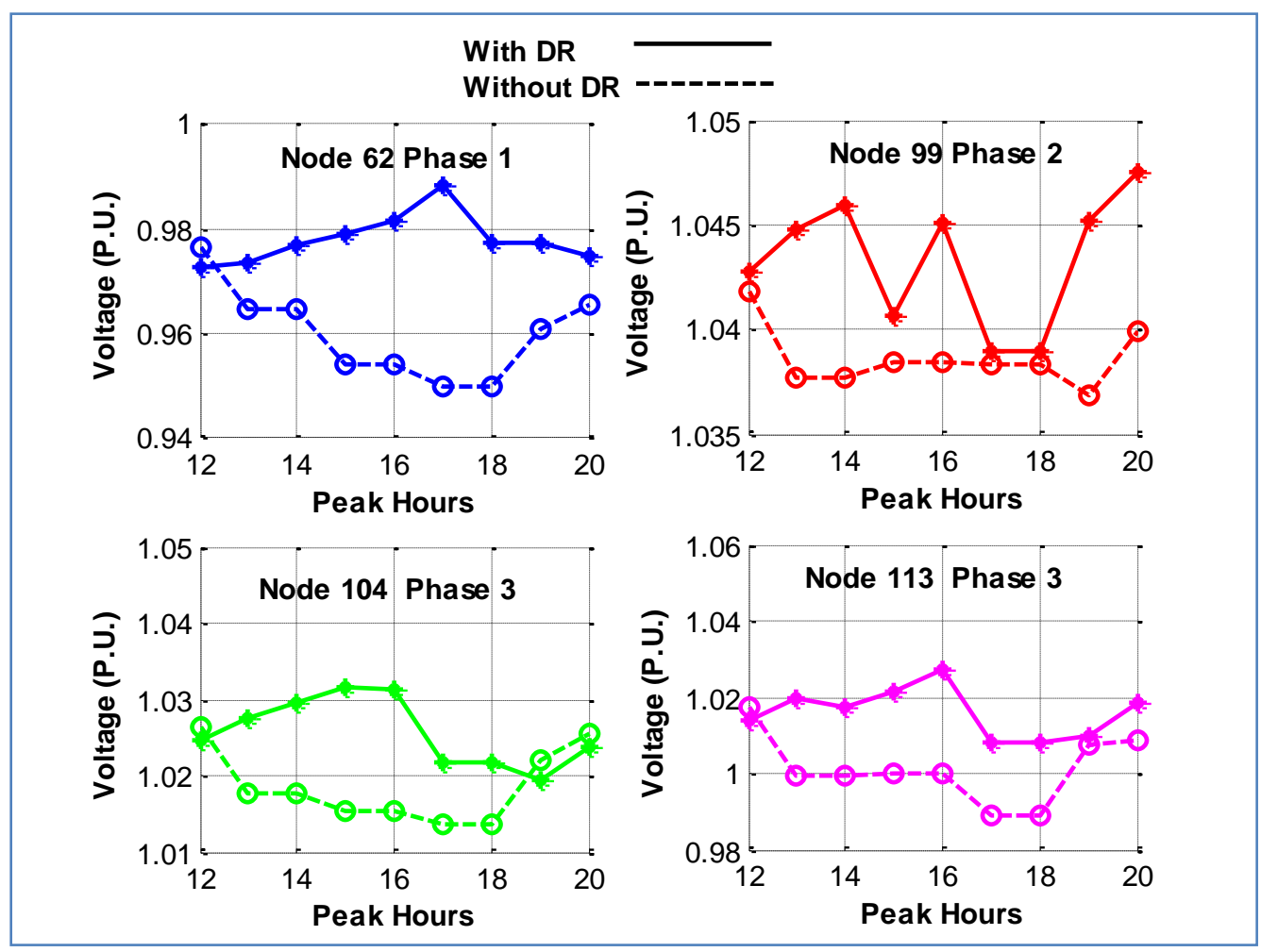

Figure 5-5: Comparison of Voltage profile of system nodes with and without DR

\subsection{IEEE 8500 Node Test Feeder}

The IEEE 8500 node test feeder [61] as shown in figure 5-6 represents a large radial distribution feeder that includes many elements that form integral part of North American MV distribution feeder: multiple feeder regulators, per-phase capacitor control, feeder secondaries, and service transformers. The 8500 node test feeder provides an excellent platform for various load shape studies and volt/var simulation. The feeder consists of residential loads, each connected to a $120 \mathrm{~V} / 240 \mathrm{~V}$ split-phase transformer. This kind of residential loading through split phase service transformer (shown in figure 5-7) is a unique feature of the feeder. Such a residential loading configuration makes it convenient and more meaningful to show the impact of residential DR models on the feeder. Impacts of various DR models on the system are analyzed on a section of the circuit as shown in figure 5-6 has been chosen for analyzing the impacts of various DR models on the system. This section consists of 245 individual residential loads and contributes around 20 percent of the total system load. 


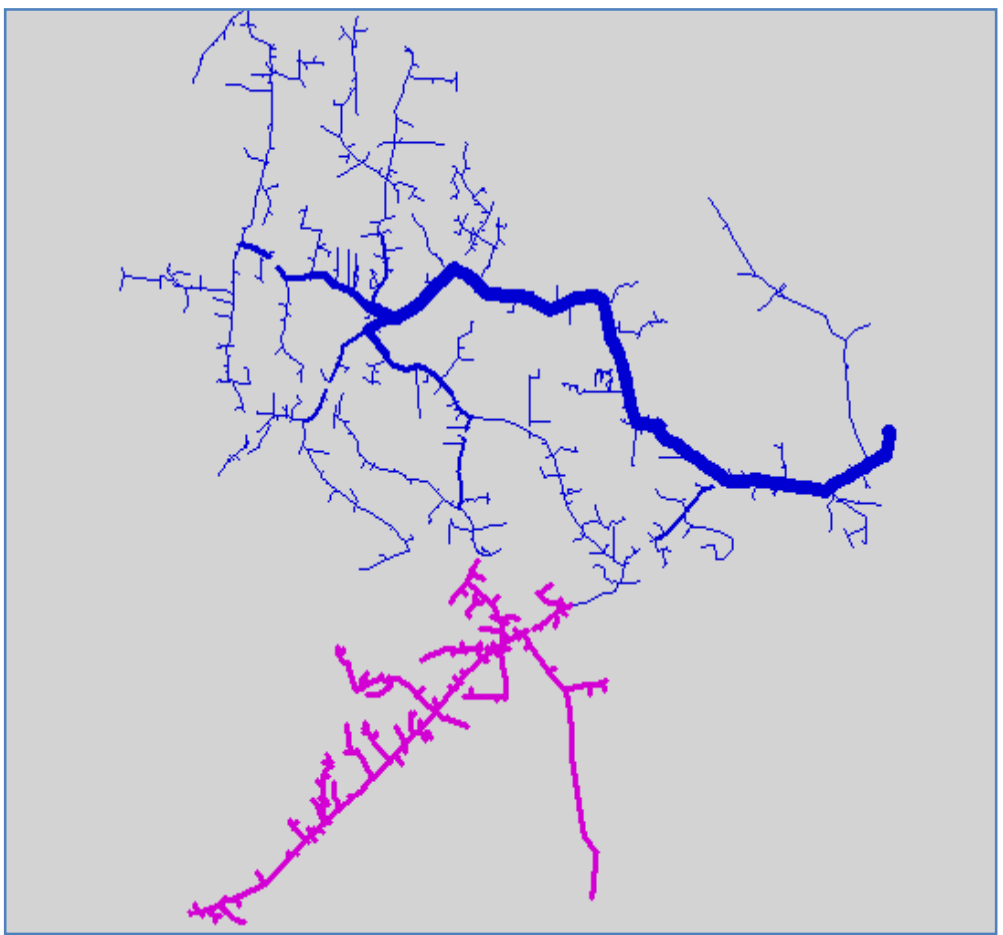

Figure 5-6: IEEE 8500 node test feeder showing section with DR loads

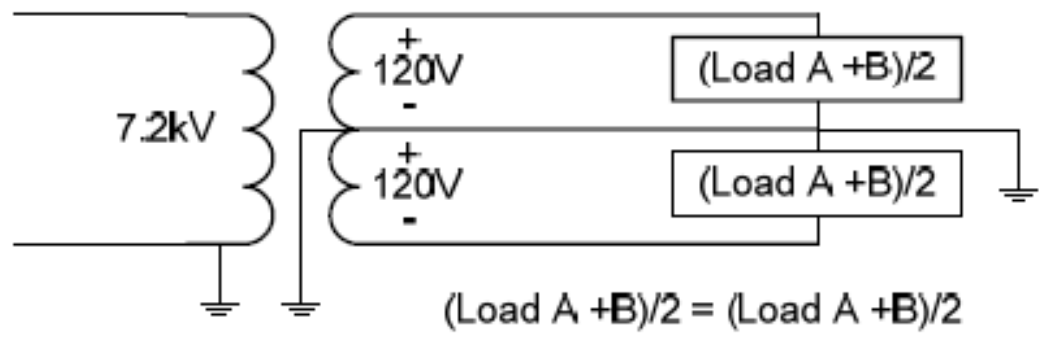

Figure 5-7: IEEE 8500 node test feeder - Split Phase Transformer and Residential Load Configuration 
The test system was simulated in OpenDSS for studying the effects of DR on the test feeder. Time series distribution power flow is run for five different scenarios.

- Scenario 1 - No Demand Response: This is the base case distribution power flow with 24 hour residential load shape base loads in the section of interest.

- Scenario 2 - LR Consumers: In this case, the loads of the highlighted section are modeled as LR consumers by assigning a DR pattern derived from the PEM of LR consumer.

- Scenario 3 - SR Consumers: In this case, the loads are assigned a DR pattern derived from the PEM of SR consumer.

- Scenario 4 - RW Consumers: In this case, the loads are assigned a DR pattern derived from the PEM of RW Mixed consumer.

- Scenario 5 - Mix of LR, SR and RW Consumers: In this case, each consumer type LR, SR and RW Mixed constitutes one third of the total section load.

\subsubsection{Voltage Analysis Results}

The voltage analysis of the system with DR integrated into the highlighted section yields many interesting results. As expected, all the monitored secondary nodes of the section showed improved voltage profile for scenarios 2, 3, 4 and 5 during high price periods. It was observed that DR during peak hours boosts the node voltages which would otherwise sag. Figures 5-8 and 5-9 show voltage comparisons at one of the load end nodes, (SX3047289A) of the feeder for the various test scenarios. As seen from figure 5-8, the node voltage during peak pricing hours drops below 0.95 p.u. for the base case without any demand response. However, with DR loads in the system, a considerable boost in voltage above 0.95 p.u. was observed for all the other scenarios. A closer look at figure 5-9 shows the redistribution trend with LR consumer during off peak hours. As seen from the figure, most of LR consumer's load is shifted to these early hours of the day due to which voltage for LR consumer dips as compared to any other consumer type. Since a RW consumer shifts load over a range of 5 hours, there is some voltage dip from hours 6-12 after which peak pricing starts. Voltages of SR consumer are constant for these hours and for scenario 5 (mix of all consumer patterns), voltages lie between voltage profiles of SR and LR consumers. 


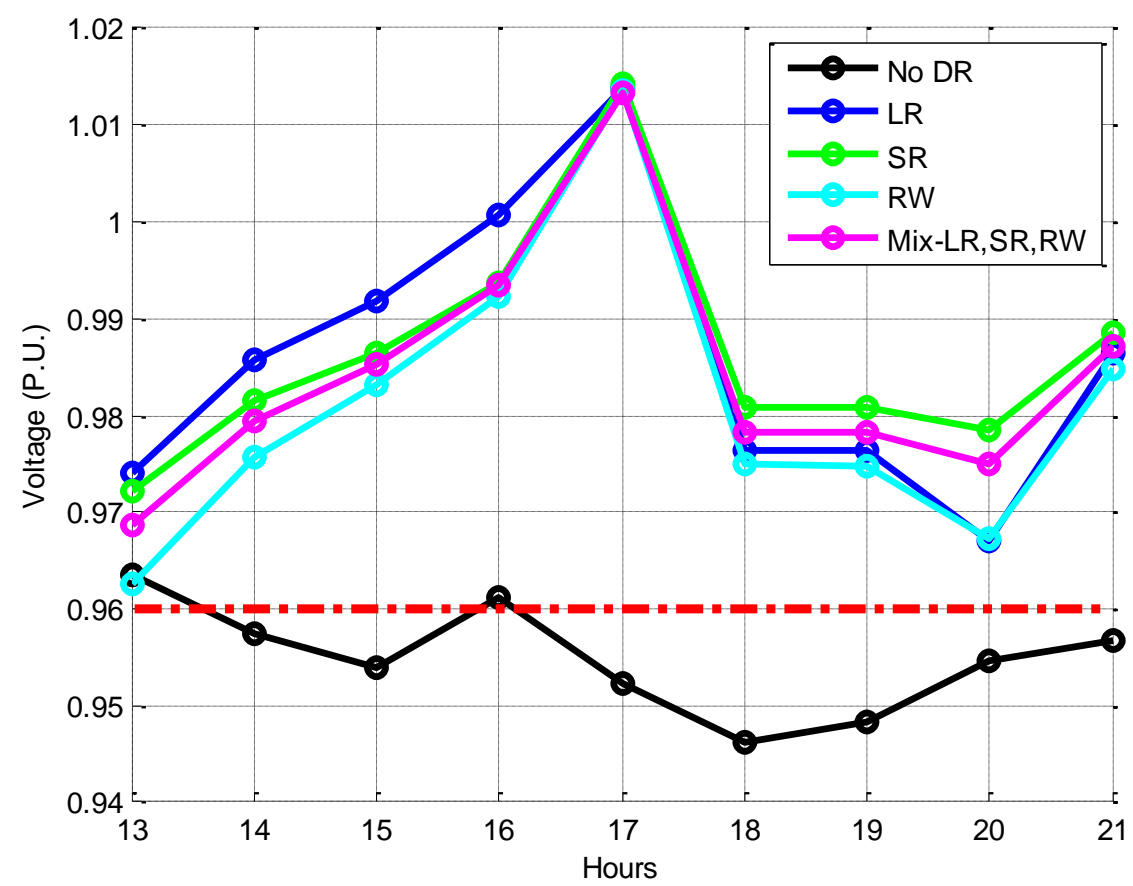

Figure 5-8: Voltage profile at node SX3047289A-Phase 1 during peak pricing hours

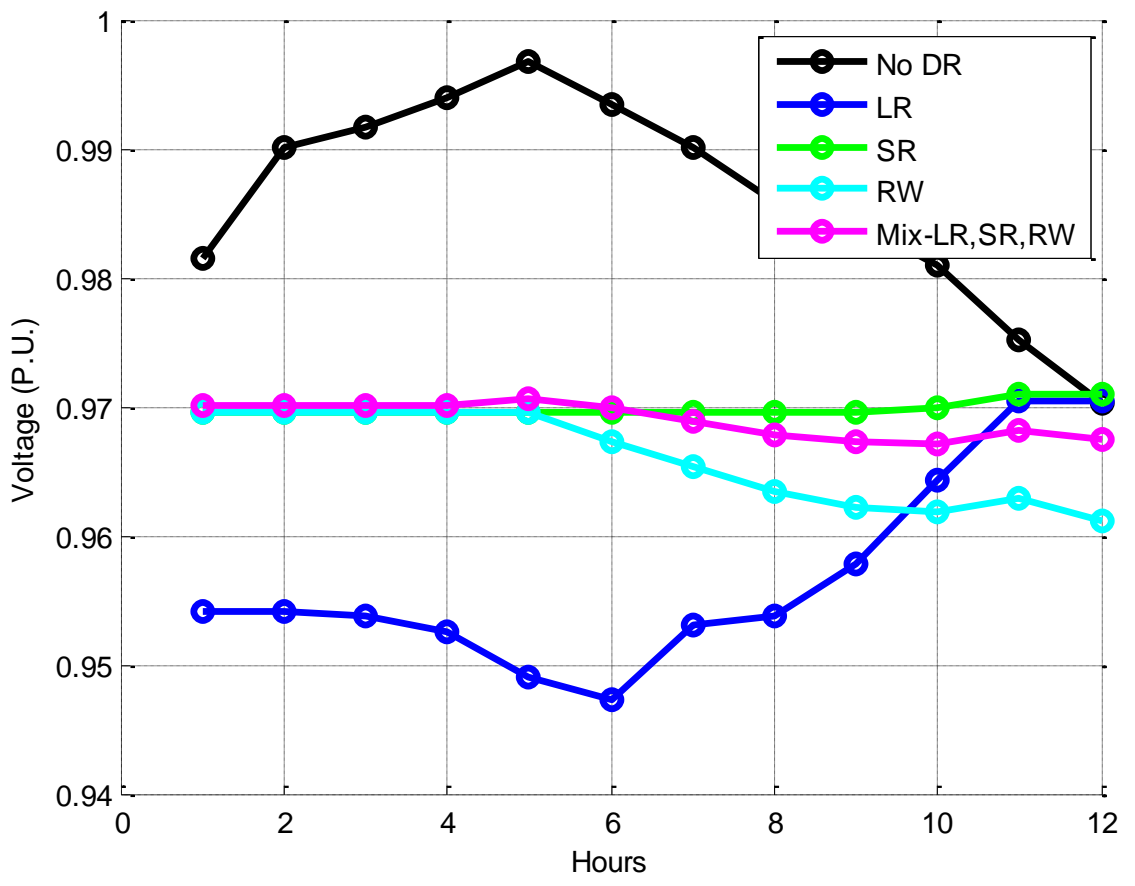

Figure 5-9: Voltage profile at node SX3047289A-Phase 1 during off peak hours

Figure 5-10 examines the voltage profile at another secondary node (SX3029498C) for all the consumer types. Sharp rise in the voltages is observed during peak hours as a result of vigorous DR measures by all the consumer types. At instants when voltages of no DR case are over 0.98 p.u., an additional boost of 0.1 
to 0.25 p.u. is seen with DR enabled loads. Figure 5-11 shows voltage analysis for the same node during off peak hours.

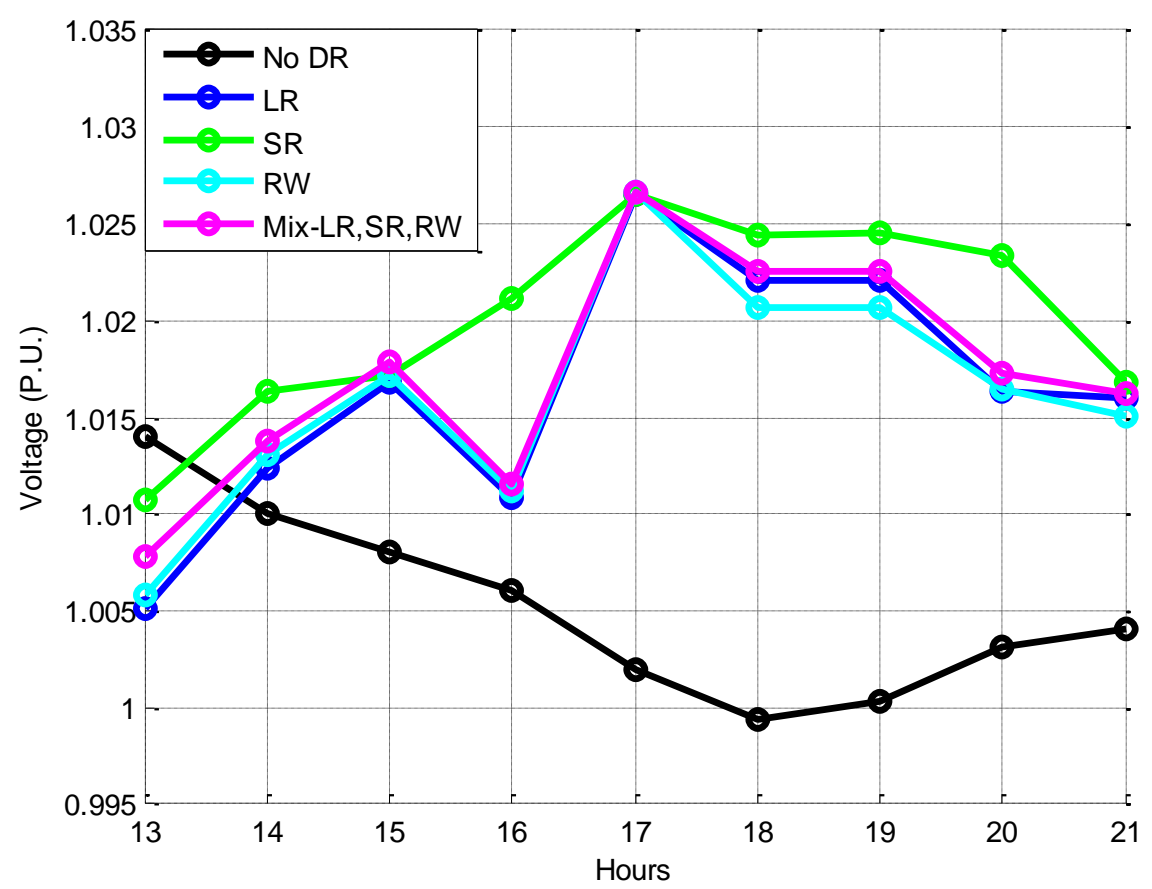

Figure 5-10: Voltage profile at node SX3029498C-Phase 1 during peak pricing hours

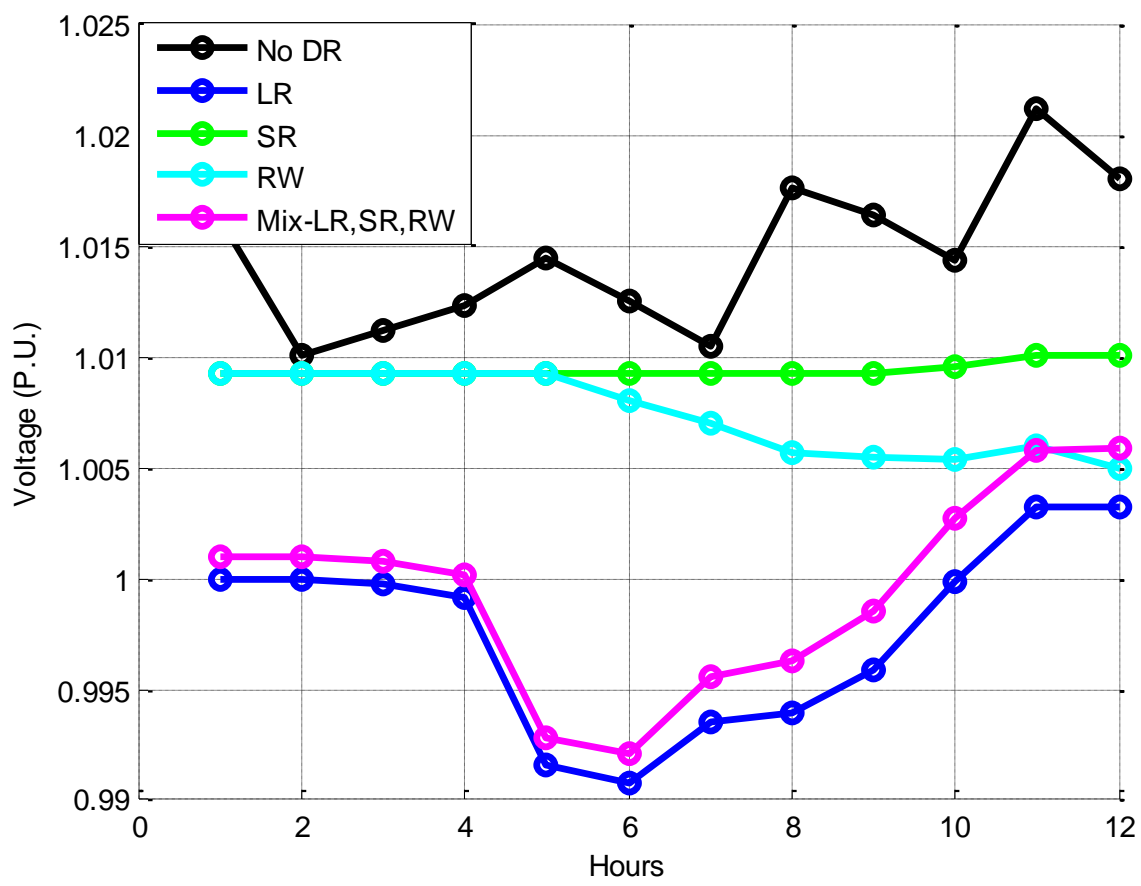

Figure 5-11: Voltage profile at node SX3029498C-Phase 1 during off peak hours 


\subsubsection{Loss Analysis Results}

The KWh losses for the section of interest were analyzed as a percentage of the total feeder loss over the period of 24 hours for different consumer type scenarios. For scenarios with responsive loads, tremendous reduction in losses was observed for the peak pricing periods as shown by the surface plot of figure 5-12. For the No DR case, losses are at maximum during peak hours. However, for other customer types, the percentage losses are considerably less. At hour 17, the percentage losses for LR, SR and RW consumers dip to as low as 4 percent as against 15 percent for the case without demand response. Lowest percentage losses occur for SR consumer as they respond to high prices during peak hours by immediate DR. However, an LR or RW consumer sheds load partially and redistributes it to lower priced periods.

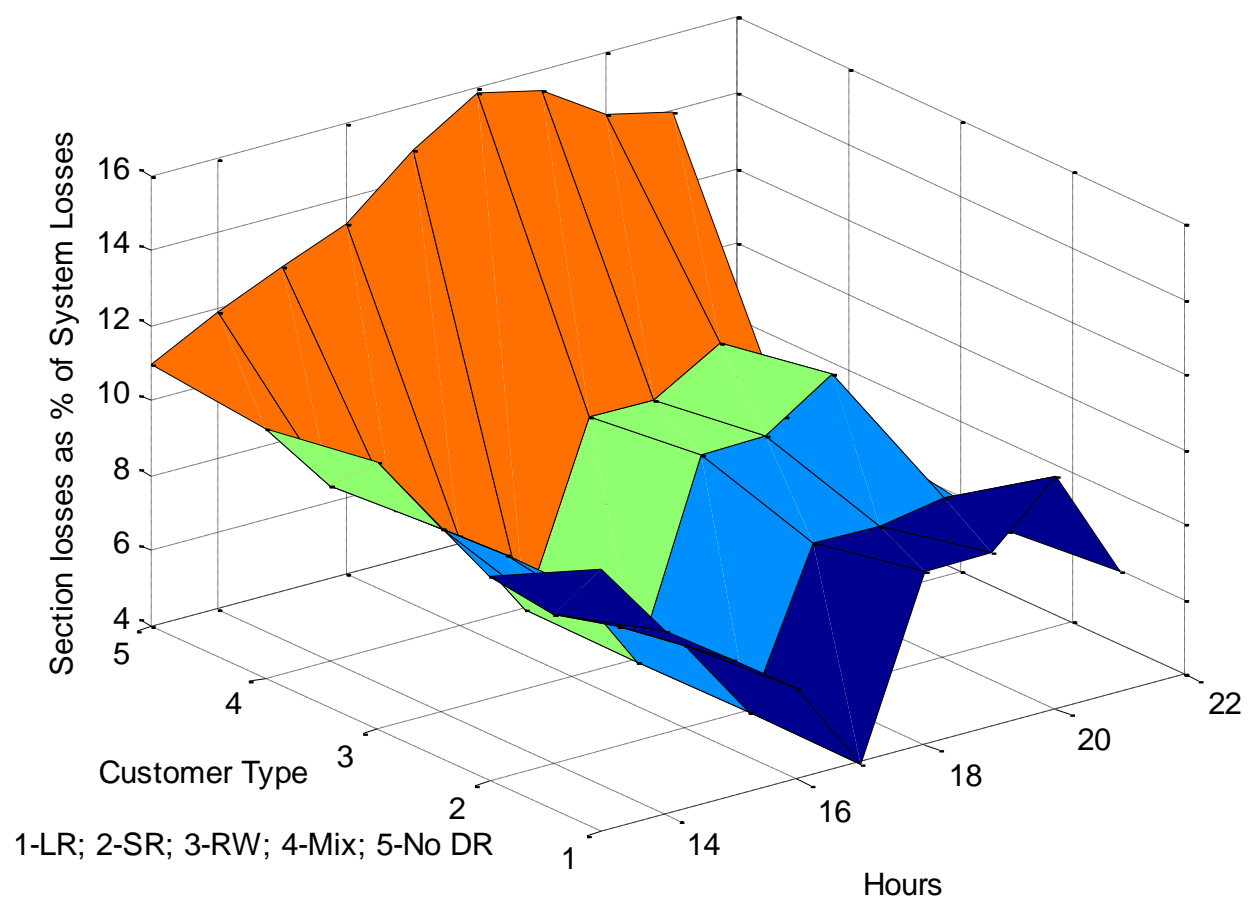

Figure 5-12: Loss Analysis of section during peak pricing hours

A similar loss analysis of the system during early hours shows that the case with no DR has minimum loss percentage as indicated by figure 5-13. Following this, SR, RW and Mix consumers have a slightly higher loss percentage due to increased consumption due to lower prices. LR consumer records the maximum percentage losses during early hours of the day. LR consumers, owing to their optimizing nature, shift loads throughout the day and their consumption is maximum during low priced periods resulting in more losses than any other consumer type. Figure 5-14 shows the sectional losses in KWh for various scenarios over an extended period of time that includes both peak and off peak pricing hours. It can be observed that the sectional losses for the No DR case gradually decrease as we move from peak 
loading (or pricing) hours to valley periods. A reverse effect is observed with DR implemented since consumption shifts to these valley periods. This increase in losses is an indication of more consumption during off peak hours giving way for valley filling.

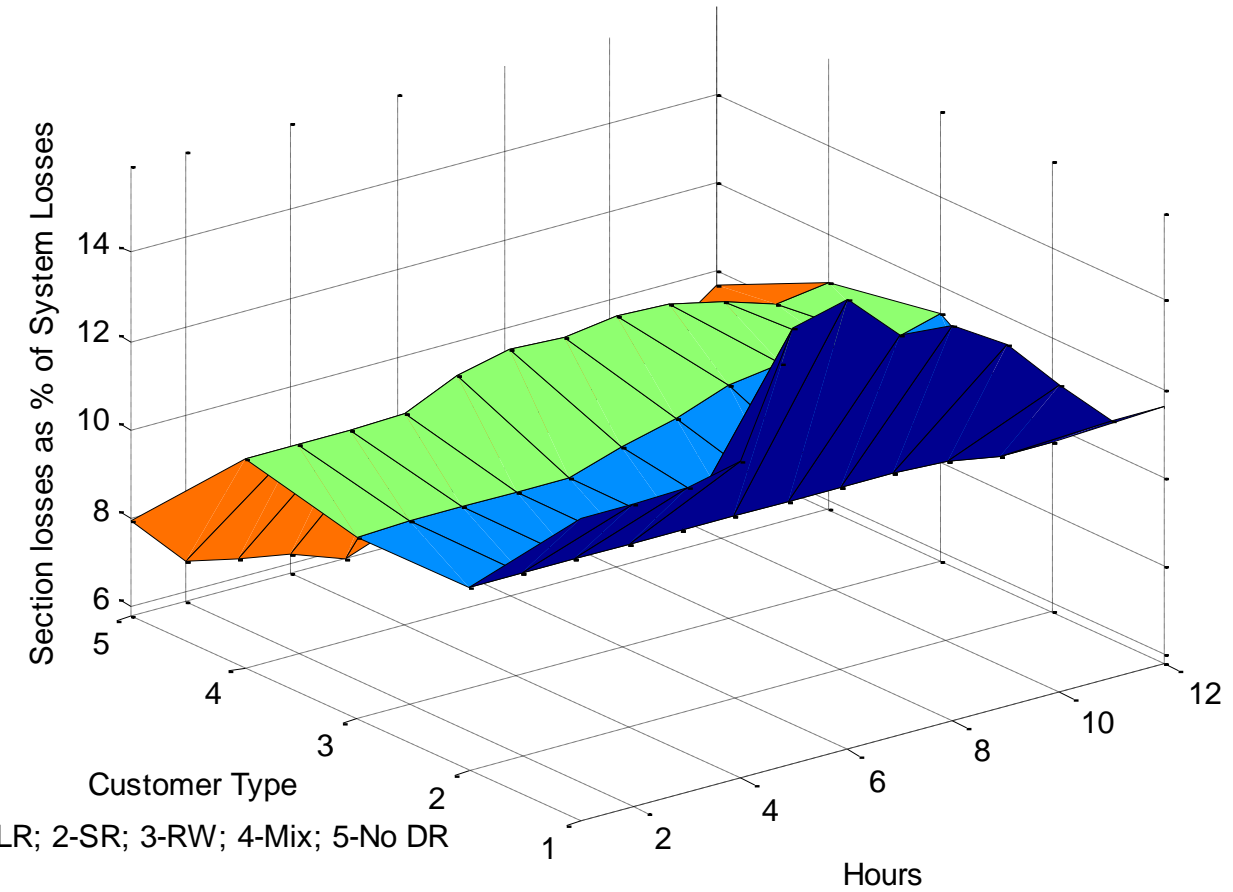

Figure 5-13: Loss Analysis of section during off peak hours

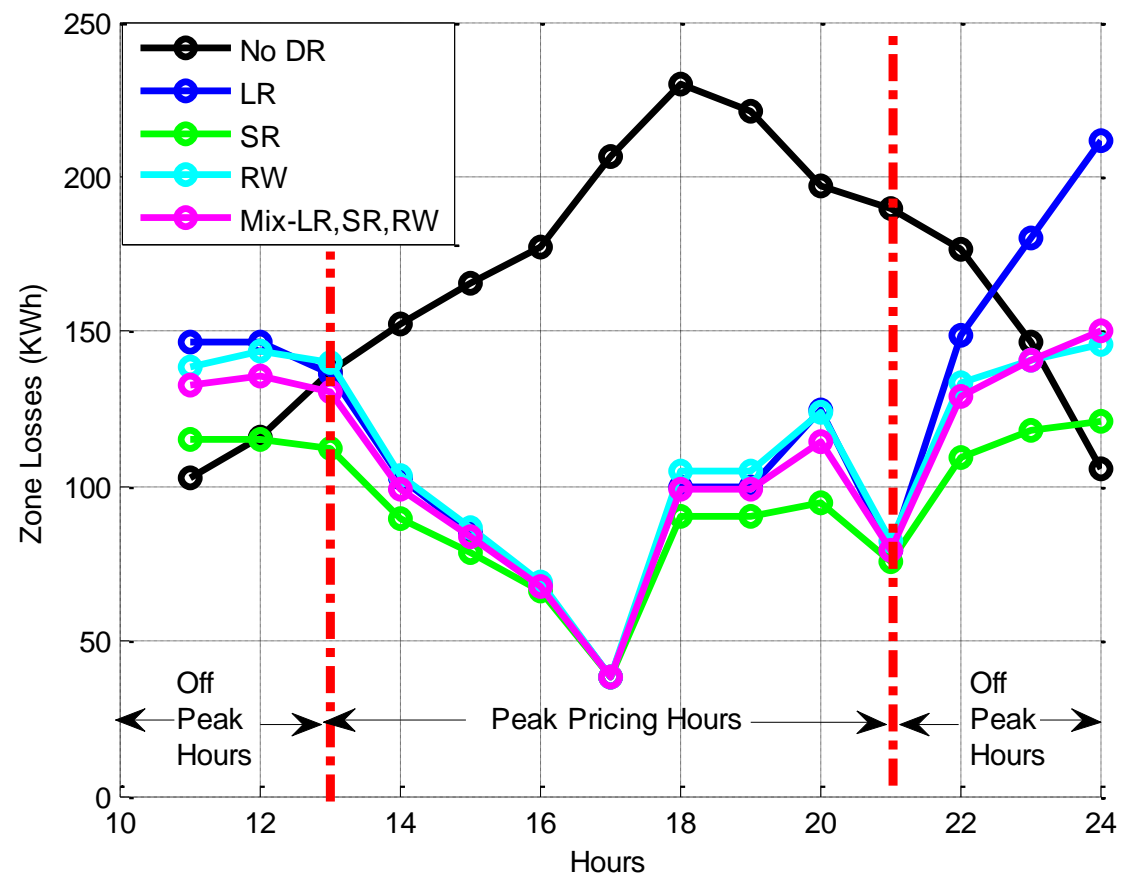

Figure 5-14: KWh Losses for the section for various DR scenarios 


\subsection{Microgrid Market Optimization}

\subsubsection{Microgrid Market Participants}

The system gross demand curve for a summer day is shown in figure 5-15. The microgrid market consists of demand side resources like wind turbine, PV system, BESS, DR loads and IPPs. Table 5-2 lists the demand side resources participating in the microgrid market along with their bidding prices.

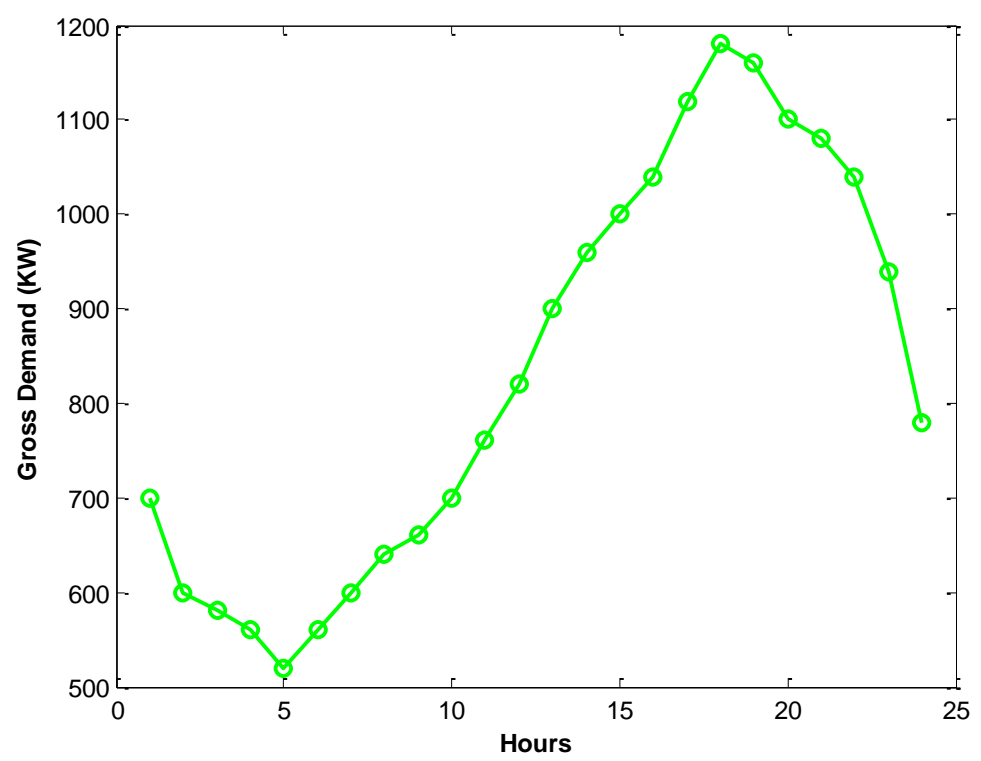

Figure 5-15: 24 Hour Gross Demand Curve

\begin{tabular}{|c|c|}
\hline Demand Side Resources & Bidding Price ( $\mathbf{c} / \mathbf{K W H})$ \\
\hline Wind & 20 \\
\hline PV & 15 \\
\hline DR Aggregator (LSE) & 25 \\
\hline IPP & 30 \\
\hline BESS & 12 \\
\hline
\end{tabular}

Table 5-2: Demand Side resources and their Bid Prices

The 24 hour availability of the intermittent sources and the IPP are to be considered by the microgrid market optimization and they are shown in figure 5-16. The specifications of the BESS used in the microgrid have been tabulated in table 5-3. 


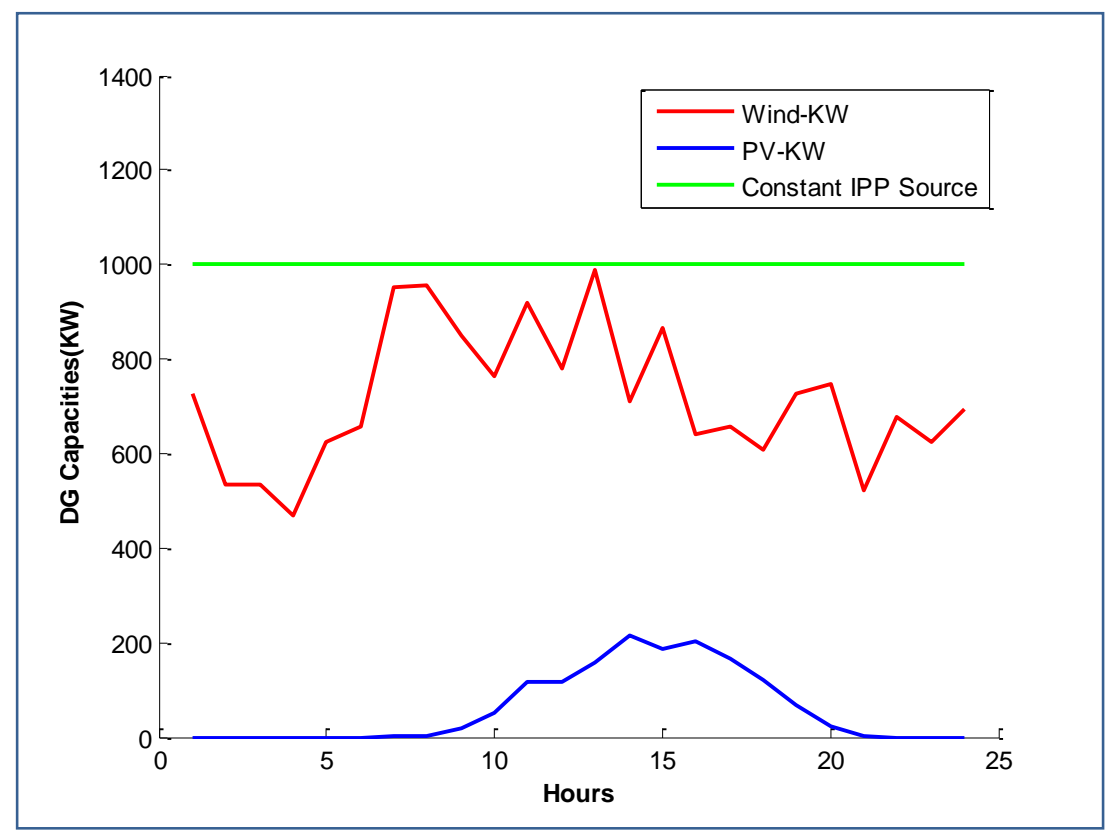

Figure 5-16: 24 Hour Availability of Wind, PV and IPP resources

\begin{tabular}{|c|c|}
\hline \multicolumn{2}{|c|}{ BESS Specifications } \\
\hline Capacity & $800 \mathrm{kWh}$ \\
\hline Power Rating & $200 \mathrm{~kW}$ \\
\hline SOC Upper Limit & $100 \%(800 \mathrm{kWh})$ \\
\hline SOC Lower Limit & $25 \%(200 \mathrm{kWh})$ \\
\hline DOD & $75 \%(600 \mathrm{kWh})$ \\
\hline Efficiency & $80 \%$ \\
\hline Terminal Voltage & $600 \mathrm{~V}$ \\
\hline Charge Rate & $200 \mathrm{~kW} / \mathrm{hr}$ \\
\hline $\begin{array}{c}\text { Discharge Rate } \\
\text { Charge/Discharge } \\
\text { Current limit }\end{array}$ & $200 \mathrm{~kW} / \mathrm{hr}$ \\
\hline
\end{tabular}

Table 5-3: BESS Specification

\subsubsection{Market Optimization Results}

The proposed LP has the dual optimization function of economics and reliability, i.e., the DERs, DR and BESS are dispatched optimally for reduced operational cost of the microgrid, thereby ensuring that supply meets demand during islanded mode of operation. LP for the BESS determines the optimum 
charge/discharge schedule of the battery based on the real time market prices with SOC and DOD constraints of the battery. The BESS is charged during the grid connected mode and discharges during the islanded mode. However, the market RTP determines the battery charging and discharging for maximizing profit. This direct relationship between the RTP signal and BESS charge/discharge as explained in subsection 3.2.2 (equation 3.6) can be seen in figures 5-17 and 5-18. As seen from these figures, the BESS charges during early hours of the day when price signal is lowest and discharges during the peak pricing hours of the day.

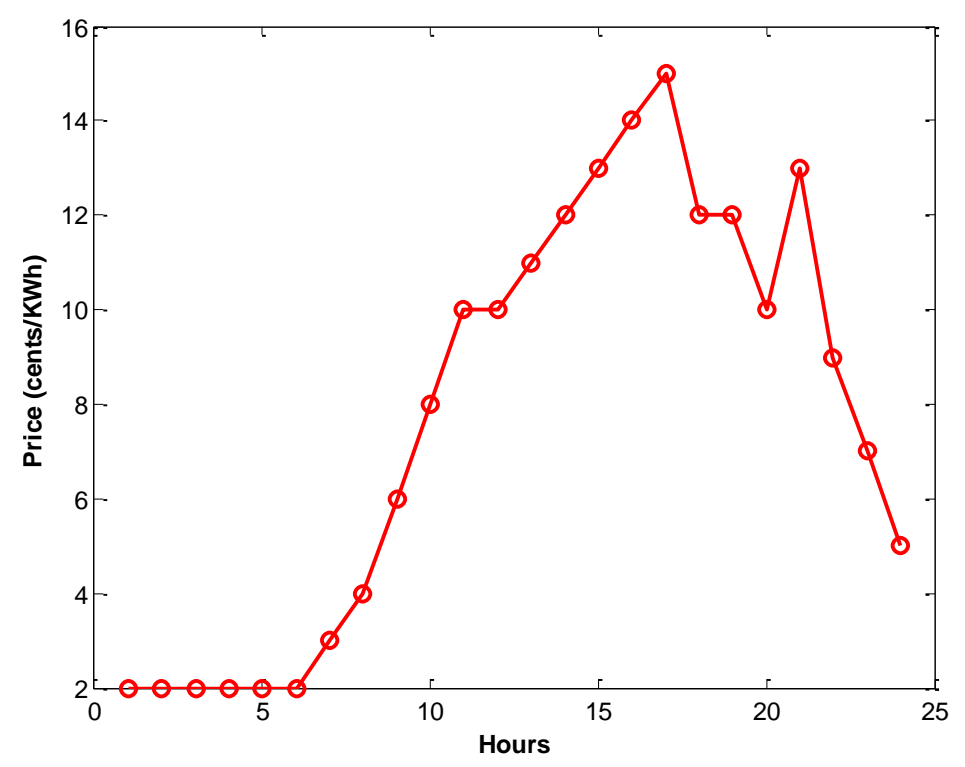

Figure 5-17: Real Time Market Prices

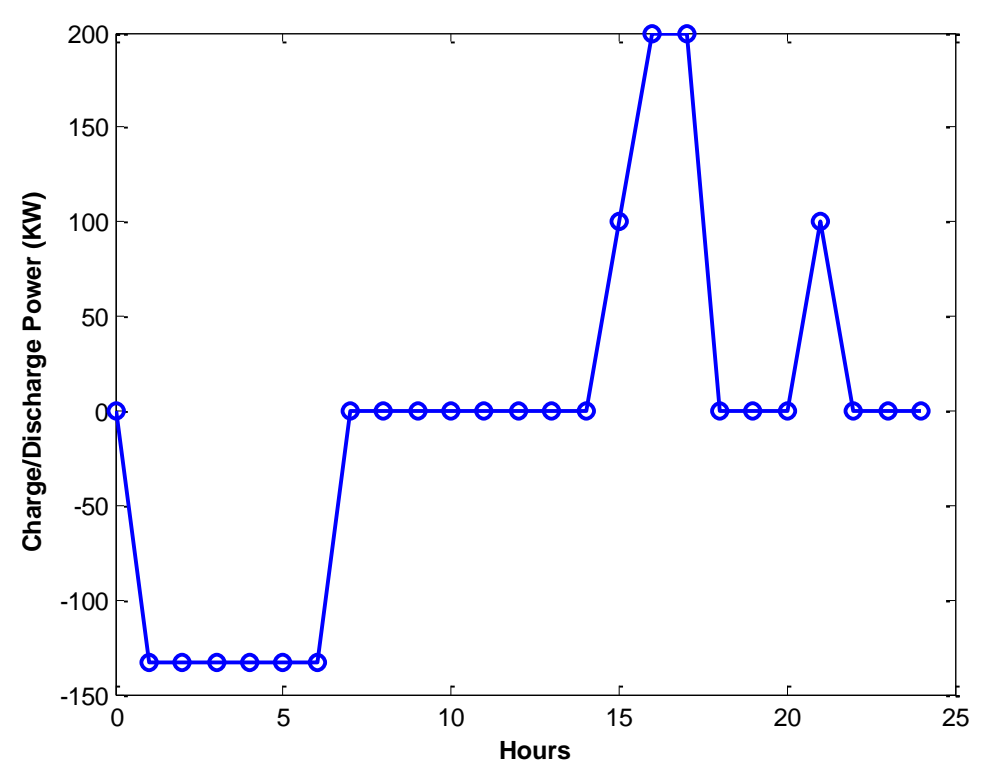

Figure 5-18: BESS Charge/Discharge Schedule 
Similar to BESS, DR loads in the microgrid are also influenced by the RTP signal. For the system gross demand shown in figure 5-15, PEM models have been applied to generate DR curves for 24 hours for all five consumer categories. The DR load curves are shown in figure 5-19.

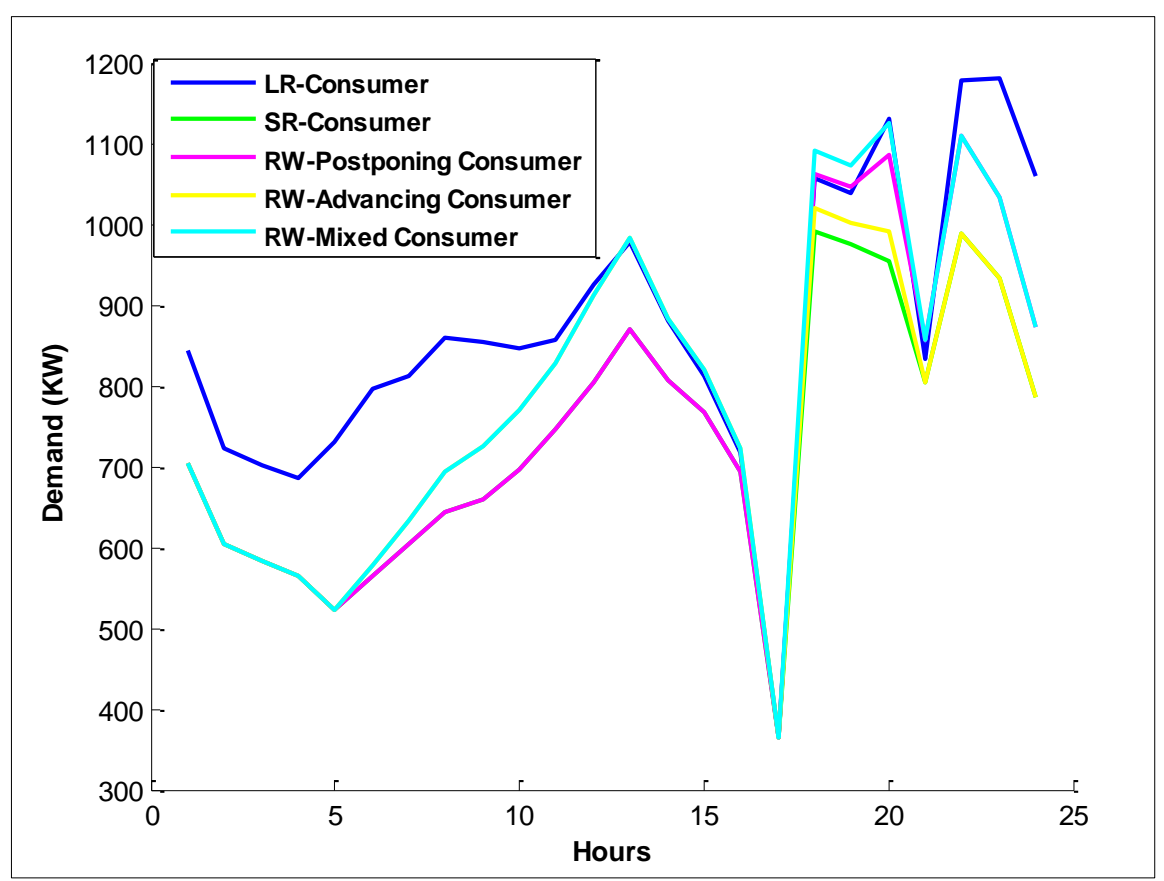

Figure 5-19: DR Patterns for Microgrid Loads

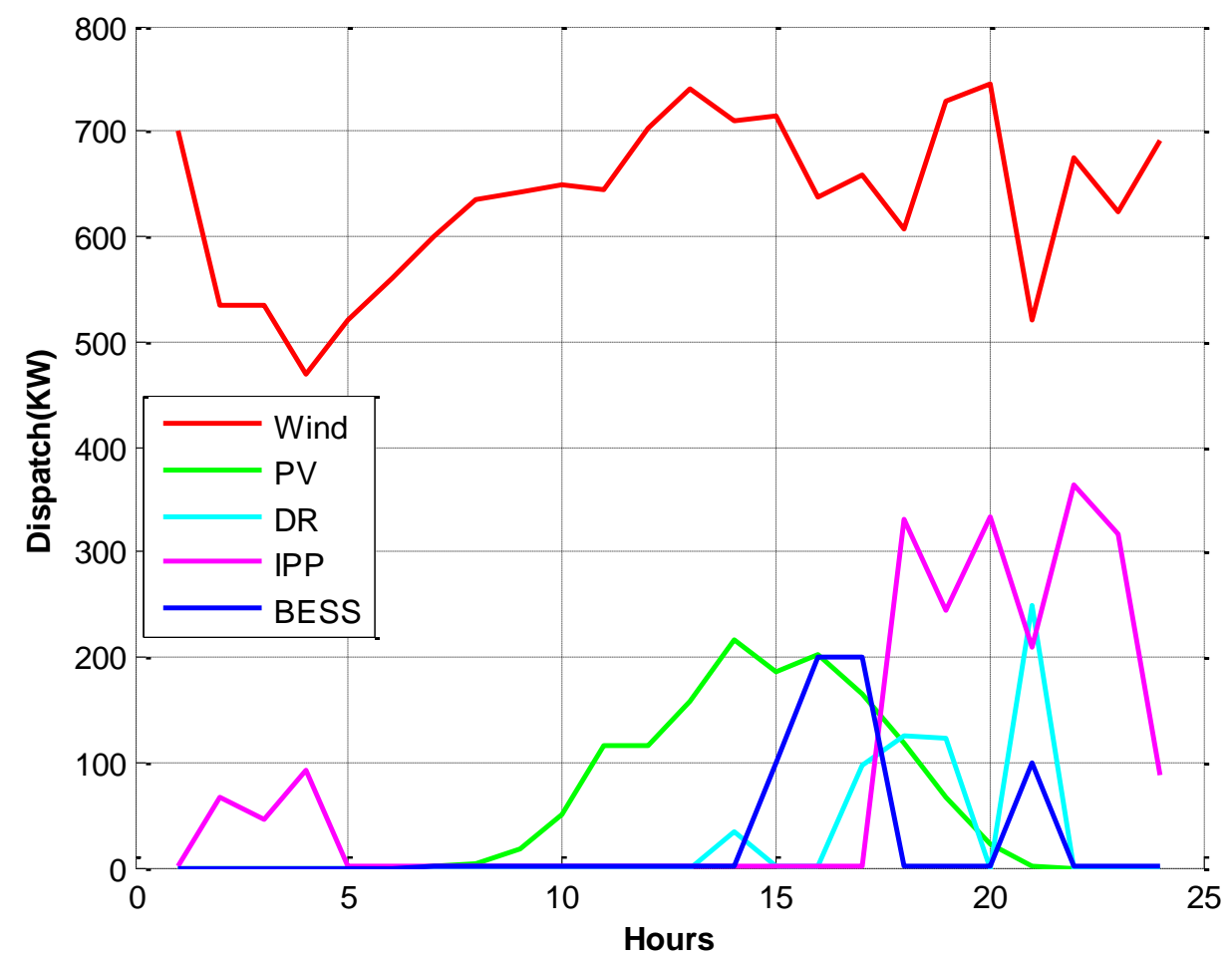

Figure 5-20: 24 Hour Optimal dispatch schedule of DR, DERs and BESS 
Figure 5-20 shows the hourly dispatch schedule of the available resources for the islanded microgrid throughout the day. It is seen that, wind and PV resources are dispatched to full capacity whenever they are available due to their lower cost. Higher cost resources of DR loads (shedding/redistribution) and Independent Power Producer (IPP) are dispatched only during peak loading hours or during unavailability of wind and PV resources. Tables 5-4 to 5-9 project some of the interesting results of the 24 hour market optimization program.

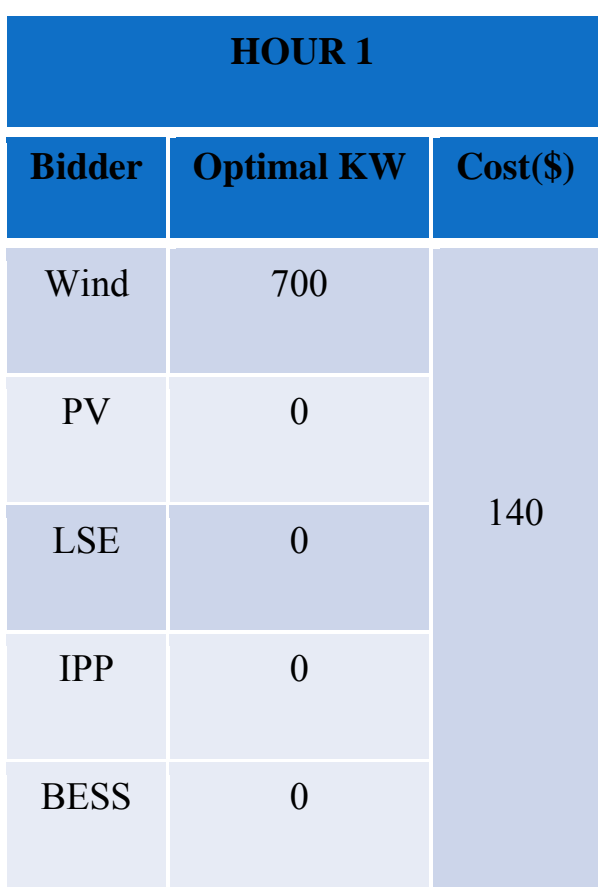

Table 5-4: Optimal Dispatch and Cost at Hour 1

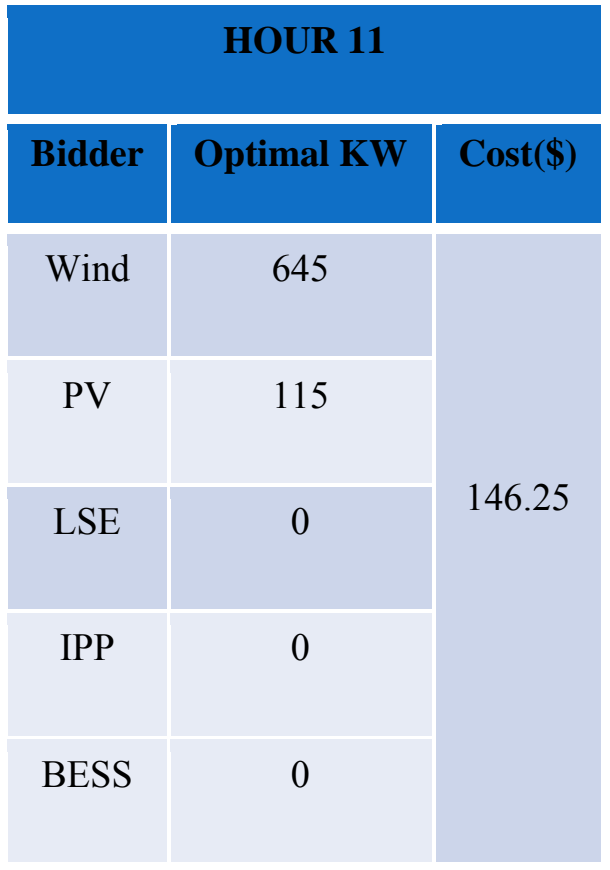

Table 5-5: Optimal Dispatch and Cost at Hour 11

- It can be seen that at hour 1 , due to reduced system load, only the wind resource is utilized.

- At hour 11, lower cost resources of wind and PV are dispatched at their maximum capacities. It is also observed that during these low load periods, the operating cost of the microgrid is reduced to the dispatch of the least expensive resources.

- As we move to hour 14, the load increases further and now responsive DR loads are shed by the LSE in addition to wind and PV resources so that the total system demand is still sustained and due to the higher bid prices of LSE, the overall microgrid cost rises. 


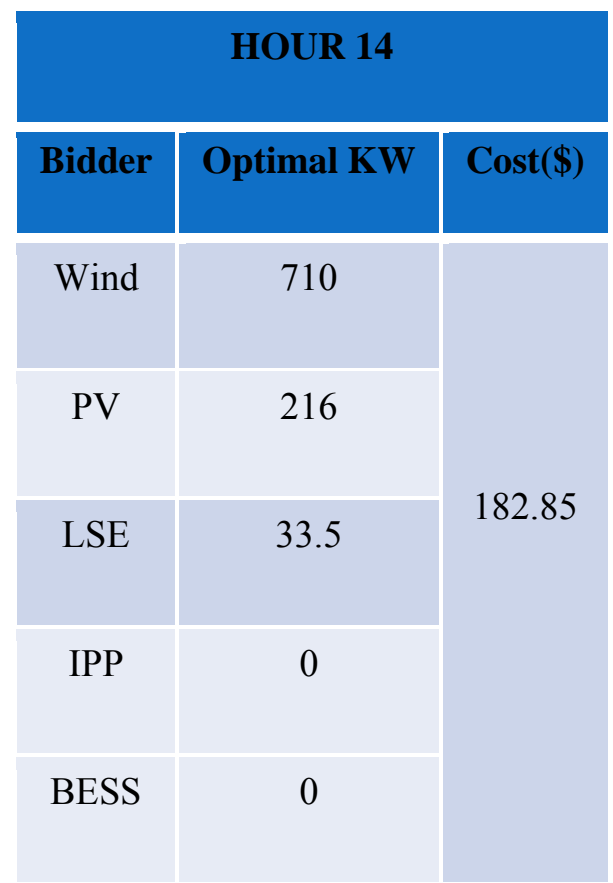

Table 5-6: Optimal Dispatch and Cost at Hour 14

\begin{tabular}{|c|c|c|}
\hline \multicolumn{3}{|c|}{ HOUR 18 } \\
\hline Bidder & Optimal KW & Cost(\$) \\
\hline Wind & 606 & \\
\hline PV & 119 & \\
\hline LSE & 124 & 269.35 \\
\hline IPP & 330 \\
\hline BESS & 0 \\
\hline
\end{tabular}

Table 5-8: Optimal Dispatch and Cost at Hour 18

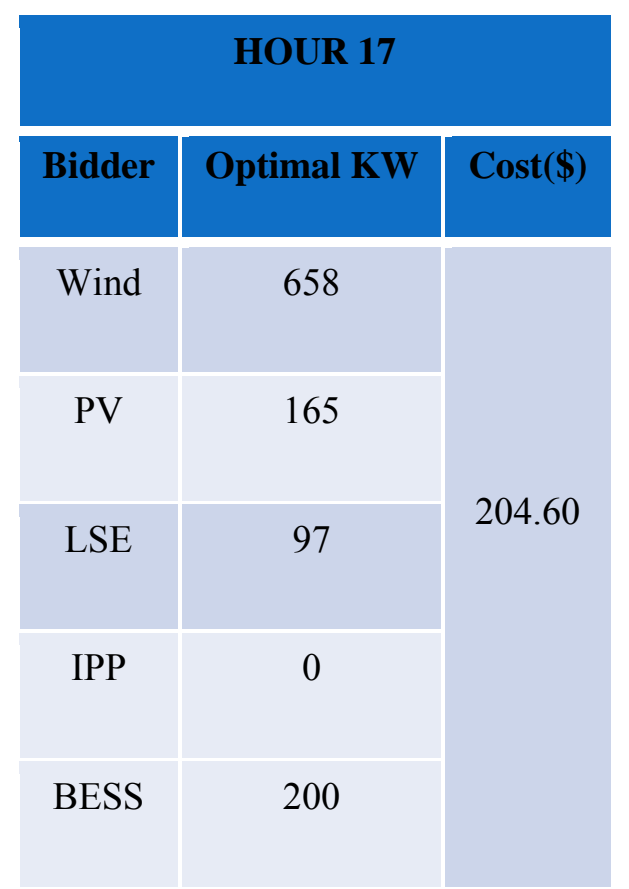

Table 5-7: Optimal Dispatch and Cost at Hour 17

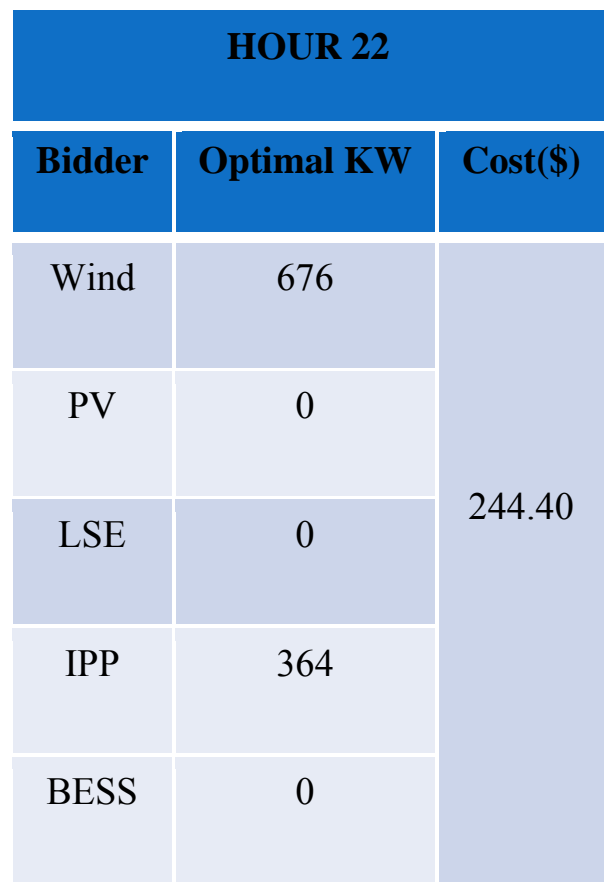

Table 5-9: Optimal Dispatch and Cost at Hour 22 
- At hour 17 it is seen that the gross demand of the islanded microgrid system is at its peak. All the available resources are dispatched in the most economical way possible. BESS, being a low cost resource is in its discharge cycle during this hour (as determined by the LP for determining optimal charge/discharge) and it is dispatched at its maximum capacity. Volume of DR by LSEs has also increased to meet the load.

- Hour 18 is again a peak loading period. It can be observed that the IPP supplies a huge portion of the load during this hour due to the unavailability of BESS energy. Since IPP bids at the highest price, operating cost at this hour is quite higher than the cost at hour 17.

- Hour 22 is another high cost period since PV resources are no longer available. Wind and IPP supply the required system demand. DR resources are not available during this hour due to the reduced market prices.

\subsubsection{Microgrid Simulation}

OpenDSS was utilized for time series simulation of the unbalanced microgrid network. IEEE 123 node radial feeder which was described in detail in section 5.1 is modified as an islanded microgrid with DGs modeled as voltage sources behind specified reactances. This was achieved by solving the basic power flow for the test system in the grid connected mode. Then the solution mode was switched to dynamics thereby opening the switch that connects the utility grid. The demand side resources like wind generator, PV, BESS and IPPs were modeled with the real power as obtained from the 24 hour dispatch schedule. DR patterns according to the developed PEMs were integrated into the system as load shapes. Time series distribution power flow of the simulated microgrid including the demand side resources was obtained. It was observed from the voltage analysis that the proposed market optimization is capable of sustaining feeder voltages within limits during all hours of operation. The time series distribution power flow analysis results for the microgrid test system are shown in figure 5-21. The figure shows the voltages across two such laterals where DGs were integrated at the lateral head. Some of the observations made from the voltage analysis results are as follows.

- During hours of low to moderate system demand (hours 1, 10 and 16), voltages as high as 1.03 per unit were maintained along the feeder till the end node.

- Since DGs were not placed at feeder ends, regular voltage drops were noticed similar to the grid connected mode of operation.

- During peak loading at hour 21, considerable voltage drops were noticed. However, the system voltages were still maintained above 1.0 per unit. 


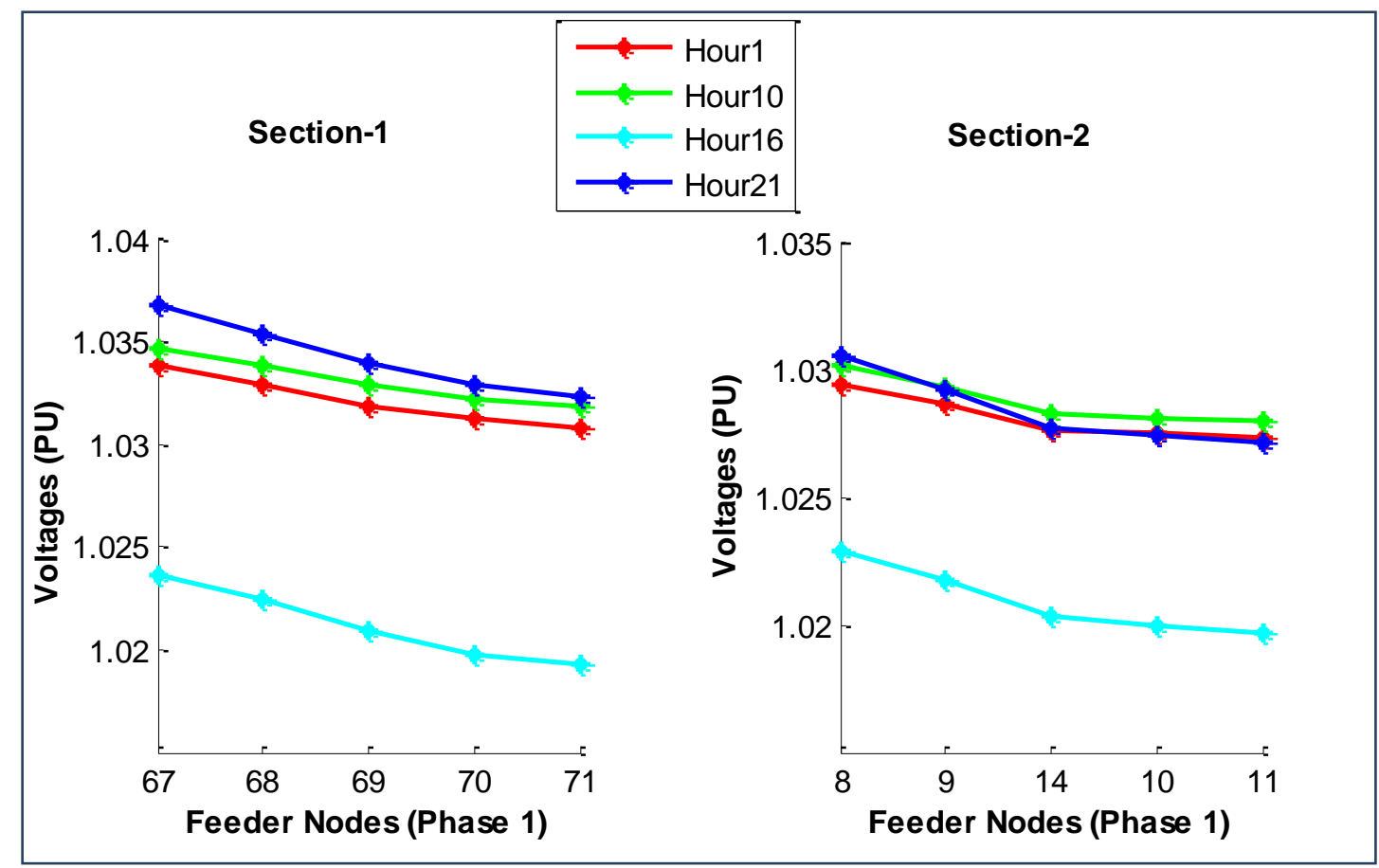

Figure 5-21: Time series voltage analysis results of microgrid with optimal dispatch schedules

\subsection{Microgrid Optimization and Simulation for 8500 Node Test Feeder}

Following microgrid simulation of 123 node test feeder, the market optimization program was scaled up to determine the 24 hour dispatch schedules for the 8500 node radial test feeder. The test feeder is supplied by a $12.47 \mathrm{KV}$ medium voltage substation modeled as an infinite source and the total system load is around 11MW. A large section of this feeder contributing around 5.6 MW of loads is modeled as an islanded microgrid as shown in figure 5-22. The Point of Common Coupling (PCC) along with the location of various demand side resources in the islanded microgrid have been shown in the figure. The islanded microgrid market consists of demand side resources like wind turbine, PV system, BESS, DR loads and IPPs. The 24 hour availability of the intermittent sources and the IPP are to be considered by the microgrid market optimization and they are shown in figure 5-23. BESS specification and charge discharge schedule used for the earlier test system were employed for this test system as well. Figure 5-24 shows the hourly dispatch schedule of the available resources for the islanded microgrid throughout the day. Similar to the 123 node microgrid optimization, wind and PV resources are dispatched to full capacity whenever they are available due to their lower cost. Higher cost resources of DR loads (shedding/redistribution) and Independent Power Producer (IPP) are dispatched only during peak loading hours or during unavailability of wind and PV resources.

The time series distribution power flow analysis results for two sections of the microgrid test system (as highlighted in figure 5-22) are shown in figures 5-25 and fig 5-26. It was observed from fig 5-25 that the node voltages of section 1 were maintained above the limits during all hours of operation including hour 18 when the system demand was at its peak. During hours 18 and 24, considerable voltage drops were in the section nodes, however the system voltages were still maintained above 0.97 per unit. In case of 
section 2, the voltage trends were different from section 1. Since section 2 branches out from a PV interconnected node, penetration levels of PV over the 24 hour period affected the voltage profile of this section. Voltages as high as 1.06 per unit were observed during hours 10, 13 and 18. During hours of low or zero PV penetration, section node voltages dropped to as low as 0.95 per unit.

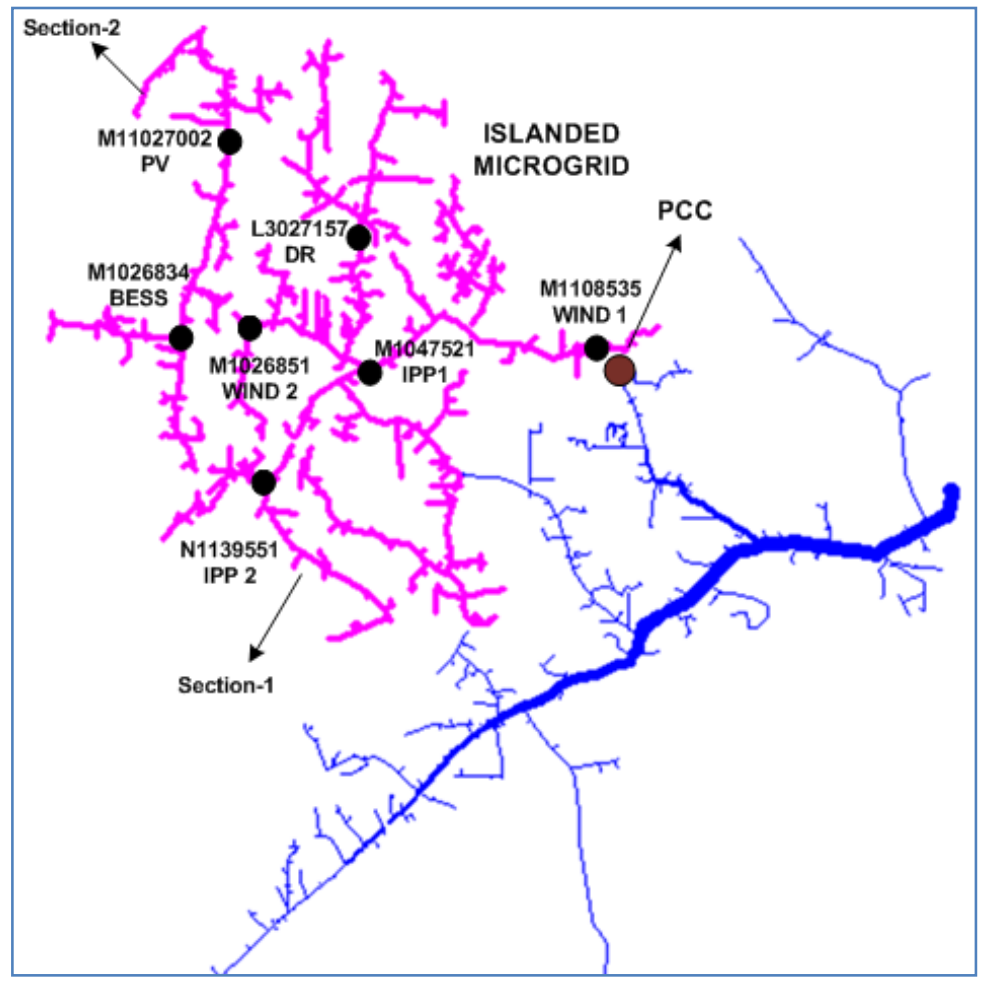

Figure 5-22: 8500 Node Test Feeder modeled as Microgrid

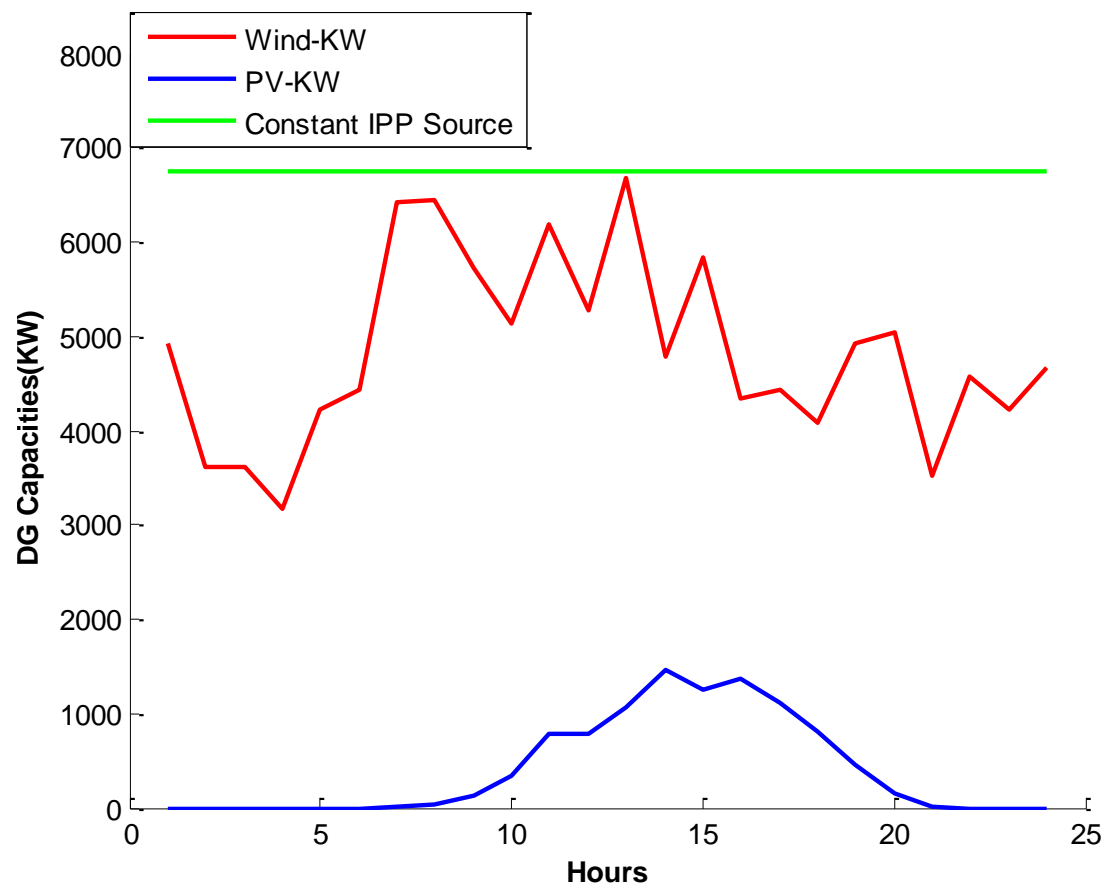

Figure 5-23: 24 Hour Availability of Wind, PV and IPP resources -8500 Node Microgrid 


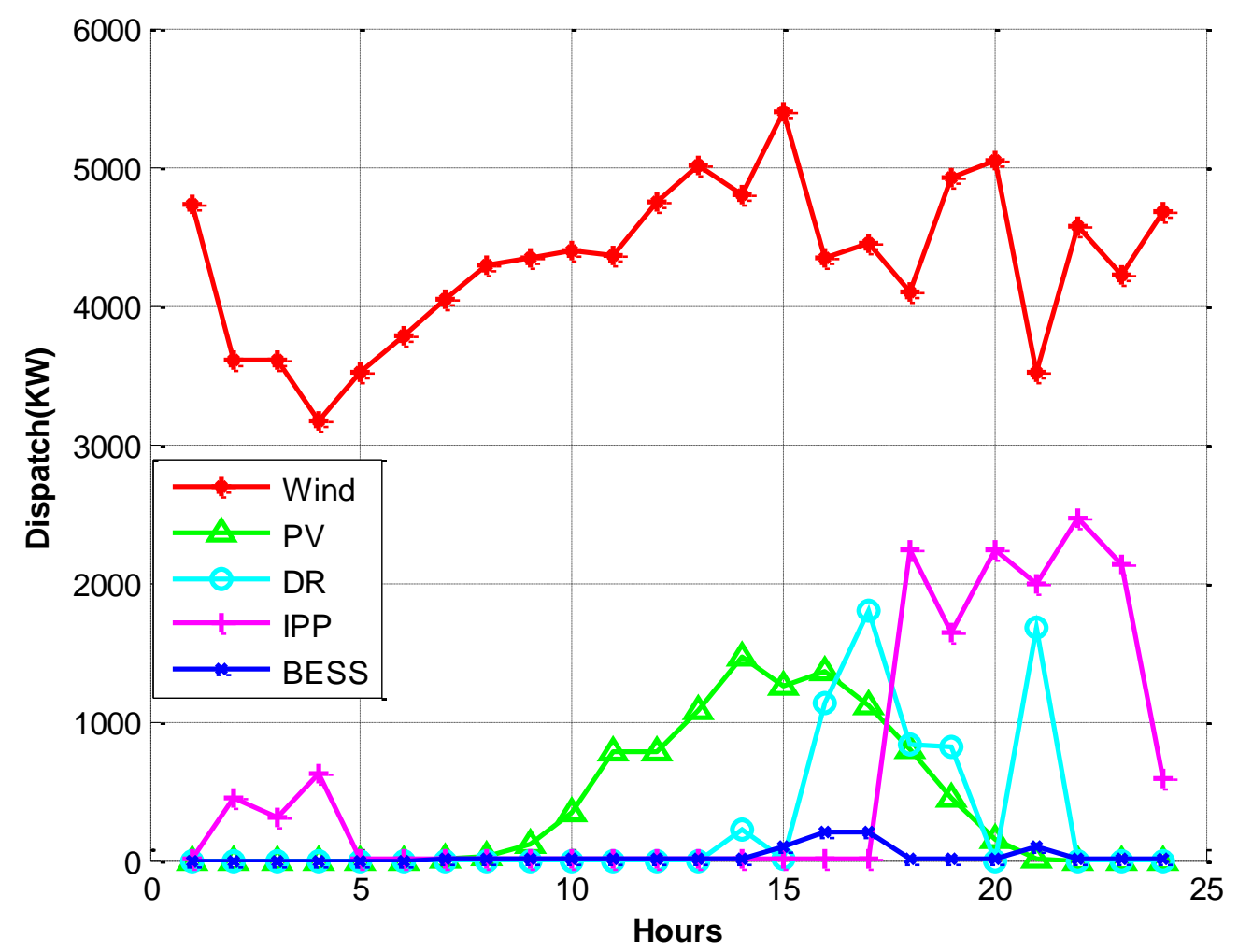

Figure 5-24: 24 Hour Optimal dispatch schedule of DR, DERs and BESS for 8500 Node Microgrid

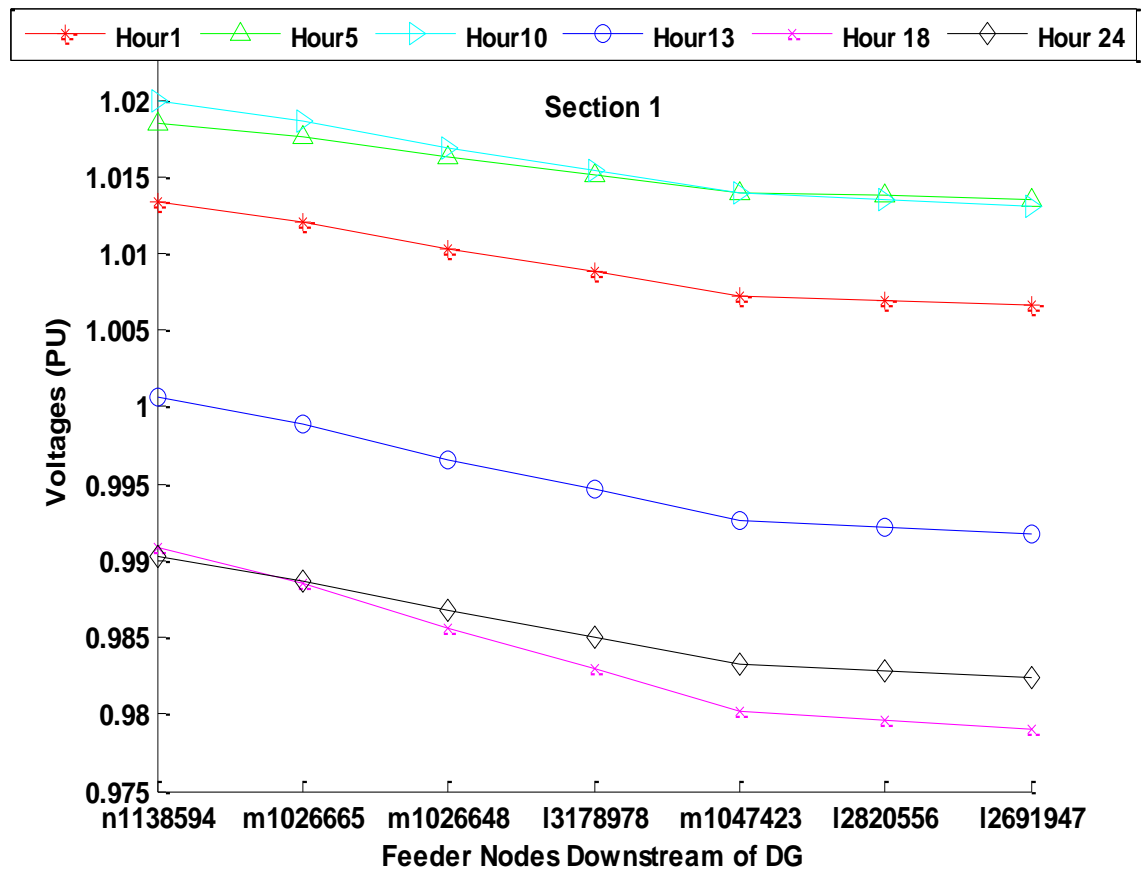

Figure 5-25: Time series voltage analysis: Section 1 


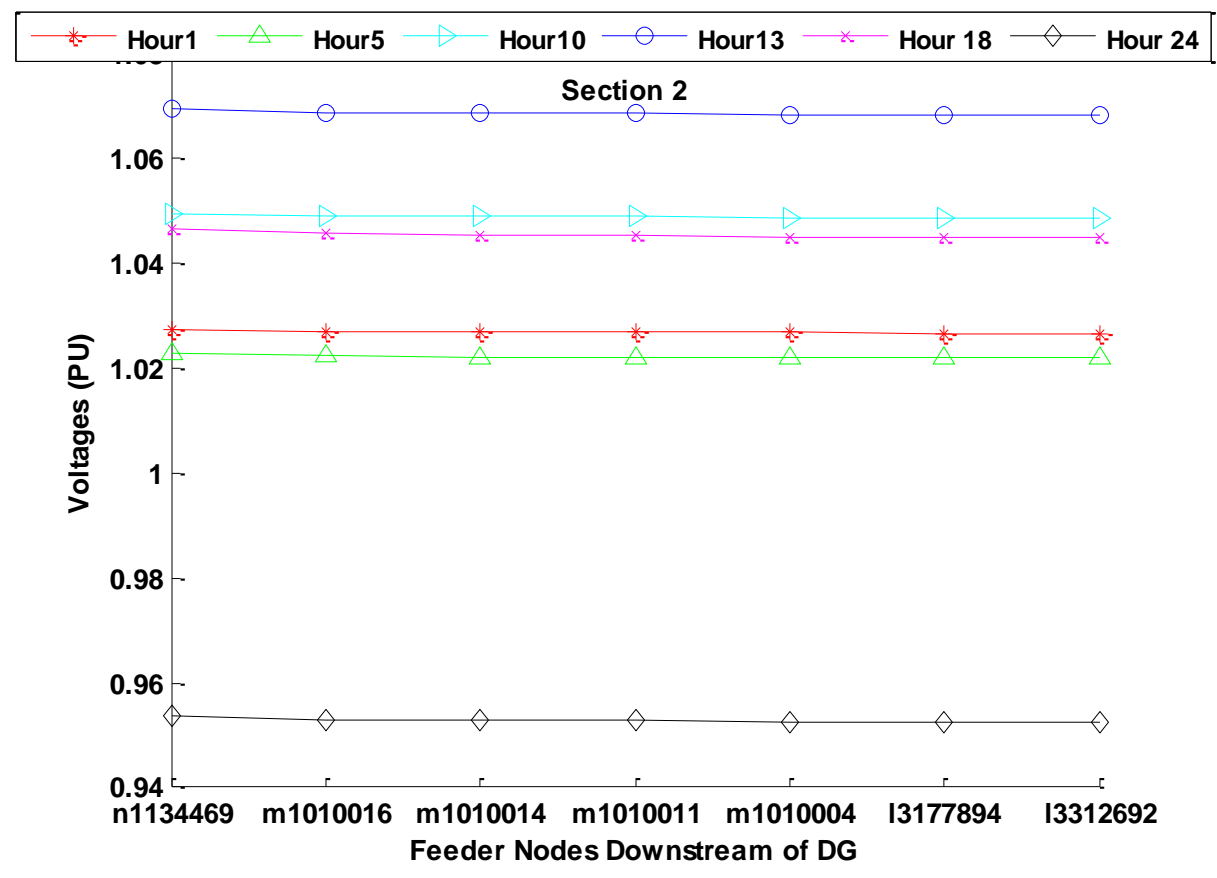

Figure 5-26: Time series voltage analysis: Section 2 


\section{Chapter 6}

\section{CONCLUSION AND FUTURE WORK}

\subsection{Conclusion}

In this thesis, some of the important demand side resources as required by a smart grid have been modeled and integrated into distribution system. Primarily, a demand response model has been developed for distribution networks. Then, this DR model has been used in addition to other demand side resources namely distributed energy resources and battery energy storage system to demonstrate a microgrid market at the distribution voltage level. The conclusions drawn from both these works have been presented in this section.

\subsubsection{Demand Response Model}

In this thesis an electricity demand response model is proposed and developed. Extensive price elasticity matrices have been developed for different types of consumers by applying economic principles to electricity consumption. These price elasticity matrices have been used to determine the level of demand response per consumer type. The developed DR models have been integrated into standard comprehensive distribution test feeders. Further, the impact of DR on system voltage and losses has been evaluated.

Results indicate that DR impacts the distribution network in 3 positive ways:

1. Voltage profile improvement

2. Loss minimization

3. Valley filling

Voltage analysis results indicate that DR has a great potential to boost the distribution system voltage at most of the critical nodes. Until recently, DR was only viewed as a means of curtailing demand side load during peak hours. However, with advancement in smart grid technologies and advanced metering infrastructure, popularly called as smart meters, there is an excellent scope for integrating DR with demand side volt/var control. This coordination can yield huge profits to utilities and consumers if applied appropriately during peak hours. 


\subsubsection{Microgrid Market Optimization}

A market optimization model has been formulated in this thesis for energy settlement in a microgrid system operating in its islanded mode. As a novel attempt residential demand side bidding through DR aggregator (load serving entities) has been incorporated into the microgrid market optimization. This has been done by utilizing the comprehensive DR models that have been developed in this thesis. BESS model has been developed to react to real time price signals and included into the market optimization. The feasibility of the proposed market optimization has been verified by a 24 hour voltage analysis on a simulated large microgrid network.

As more and more microgrids are evolving in real world, these kinds of studies help to improve the efficient operation of microgrids and minimize their cost. The role of load serving entities is an interesting area to be analyzed by including multiple LSEs with different DR patterns and PEMs as competition among LSEs can help bring down the cost of microgrids. Participation of residential consumers in demand side bidding programs could lead to optimum, efficient and economic functioning of the microgrid market.

\subsection{Future Work}

Demand Response has started gaining popularity as a multi-objective demand side management event. With the advent of advanced communication and control techniques in the grid, DR promises immense benefits to both electric utilities and consumers. Integration of Demand Response with volt/var control is a future area of research that promises tremendous benefits. A good extension of this thesis work will be the demonstration of a volt/var control algorithm that utilizes DR model and distribution operation model in real time. Maximum benefits of peak demand curtailment can be achieved through integration of DR with volt/var control algorithm. Rapid growth of advanced metering infrastructure and distribution automation (DA) measures should be able to serve the purpose of coordinating DR with various demand side management events at the distribution level. A coordinated distribution management system involving DA, DR and VVC and fault location, isolation and restoration is the need of the hour for the distribution grid. This thesis will serve as a platform for such ambitious project.

Apart from distribution system applications, DR has got a tremendous potential at the transmission level as well. DR programs such as emergency demand response can play an important role in congestion management of the transmission grid. This can even impact the market clearing price of the grid and mitigate market price spikes. In addition to transmission congestion, dynamically controlled DR can 
contribute towards grid frequency stability as well. Properly planned and executed DR programs at the ISO level can greatly help to maintain the stability and security of the grid. A thorough analysis on these impacts of DR on the transmission grid has to be studied.

Microgrid market simulation and determination of energy management strategies at the microgrid level is a rapidly growing research area. Scaling up the market optimization method proposed in this for a larger real world microgrid system is a good future project. Following this, a feasibility study should be performed by simulating the microgrid network to analyze the effects of DSB, DR, and demand side resources on system parameters namely losses and voltage profile. Another improvement that can be done to this work in the modeling aspect is to develop detailed models for various DGs namely wind, PV generator, microturbine, diesel engine, etc. and integrate them into the large microgrid network.

DSB and DR have been integrated into a microgrid market in this thesis. However, setting a standard framework for a hierarchical demand side bidding in distribution network is a more challenging task. The demand side bidding proposed for a microgrid in this work, can be explored further to achieve such a competitive bidding mechanism throughout the distribution network for more efficient and competitive operation of distribution systems. 


\section{References}

1. "Title XIII - Smart Grid, Sec. 1301, Statement of Policy on Modernization of Electricity Grid", Energy Independence and Security Act of 2007 (EISA), USA.

2. Braithwait, S.D., Eakin, K., "The role of demand response in electric power market design", Laurits R. Christensen Associates, a report prepared for Edison Electric Institute, Madison, October 2002. Available: http://www.eei.org.

3. Albadi, M.H., El-Saadany, E.F., "Demand Response in Electricity Markets: An Overview", Proceedings of the IEEE Power Engineering Society General Meeting, June 2007, pp: $1-5$.

4. http://www.ameren.com/sites/aiu/MyHome/ILChoiceResidential/Pages

5. US Department of Energy, "Benefits of Demand Response in Electricity Markets and Recommendations for Achieving Them", Report to the United States Congress, February 2006. Available online: http://eetd.lbl.gov

6. http://www.oe.energy.gov/de.htm

7. http://www.oe.energy.gov/DocumentsandMedia/1817_Report_final.pdf

8. Ribeiro, P.F., Johnson, B.K., Crow, M.L., Arsoy, A., Liu, Y., "Energy storage systems for advanced power applications," Proceedings of the IEEE, vol. 89, no. 12, Dec 2001, pp: 17441756.

9. Sutanto, D., "Power management solutions for energy management, power quality and environment using battery energy storage systems", Proceedings of the IEEE International Conference on Power Electronics and Drive Systems, 1999.

10. http://www.sandc.com/products/energy-storage/default.asp

11. http://www.energy.ca.gov/2007 energypolicy

12. Antonis, G.T., Nikos, D.H., "Centralized Control for Optimizing Microgrids Operation”, IEEE transactions on energy conversion, vol. 23, no. 1, March 2008, pp: 241-248.

13. Basak, P., Saha, A. K., Chowdhury, S., Chowdhury, S. P., "Microgrid: Control Techniques and Modeling", Proceedings of the 44th International Universities Power Engineering Conference (UPEC), Sept. 2009.

14. Chatzivasiliadis, S.J., Hatziargyriou, N.D., Dimeas, A.L., "Development of an Agent Based Intelligent Control System for Microgrids", Proceedings of the IEEE Power \& Energy Society General Meeting, July 2008. 
15. Colson, C.M., Nehrir, M.H., "A Review of Challenges to Real-Time Power Management of Microgrids", Proceedings of the IEEE Power \& Energy Society General Meeting, July 2009.

16. Caramanis, M.C., Bohn, R.E., Schweppe, F.C., "Optimal Spot Pricing: Practice and Theory", IEEE Transactions on Power Apparatus and Systems, vol. PAS-101, no. 9, Sept. 1982, pp: 32343245 .

17. Schweppe F.C., Caramanis, M.C., Tabors, R.D., Bohn, R.E., "Spot Pricing of Electricity", Boston, MA: Kluwer, 1988.

18. Schweppe, F.C., Caramanis, M.C., Tabors, R.D., "Evaluation of Spot Price Based Electricity Rates”, IEEE Transactions on Power Apparatus and Systems, vol. PAS-104, no. 7, July 1985, pp.1644-1655.

19. David, A.K., Li, Y.Z., "Consumer Rationality Assumptions in the Real Time Pricing of Electricity", Proceedings of the IEEE conference on Advances in Power System Control, Operation and Management, Hong Kong, Nov 1991.

20. David, A.K., Lee Y.C., "Dynamic Tariffs: Theory of Utility-Consumer Interaction", IEEE Transactions on Power Systems, vol. 4, no. 3, Aug 1989, pp: 904-911.

21. David, A.K., Li, Y.Z., "Effect of Inter-Temporal Factors on the Real Time Pricing of Electricity", IEEE Transactions on Power Systems, vol. 8, no. 1, Feb 1993, pp: 44-52.

22. Widergren, S.E., "Demand or request: Will load behave?" Proceedings of the IEEE Power \& Energy Society General Meeting, July2009.

23. David, A.K., "Load forecasting under spot pricing", Proceedings of the IEEE Generation, Transmission and Distribution Conference, vol. 135, no. 5, Sep 1988, pp: 369-377.

24. Khotanzad, A., Zhou, E., Elragal, H., "A neuro-fuzzy approach to short-term load forecasting in a price-sensitive environment”, IEEE Transactions on Power Systems, vol. 17, no. 4, Nov 2002, pp: 1273- 1282.

25. Yun, Z., Quan, Z., Caixin, S., Shaolan, L., Yuming, L., Yang, S., "RBF Neural Network and ANFIS-Based Short-Term Load Forecasting Approach in Real-Time Price Environment", IEEE Transactions on Power Systems, vol. 23, no. 3, Aug 2008, pp: 853-858.

26. Strbac, G., Farmer, E.D., Cory, B.J., "Framework for the incorporation of demand-side in a competitive electricity market", Proceedings of the IEEE Generation, Transmission and Distribution, vol. 143, no. 3, May 1996, pp: 232-237.

27. Zhang, L., Zha, J., Han, X., Niu, L. "Day-ahead Generation Scheduling with Demand Response", Proceedings of the IEEE/PES Transmission and Distribution Conference and Exhibition: Asia and Pacific, 2005. 
28. Kirschen, D.S., Strbac, G., Cumperayot, P., de Paiva Mendes, D., "Factoring the Elasticity of Demand in Electricity Prices", IEEE Transactions on Power Systems, vol. 15, no. 2, May 2000, pp: 612-617.

29. Wang, J., Kennedy, S., Kirtley, J.A., "A New Wholesale Bidding Mechanism for Enhanced Demand Response in Smart Grids", Proceedings of the IEEE Innovative Smart Grid Technologies (ISGT), Jan 2010.

30. Palensky, P., Kupzog, F., Zaidi, A.A., Zhou, K. "Modeling domestic housing loads for demand response", Proceedings of the IEEE Industrial Electronics, Nov 2008, pp: 2742-2747.

31. Kim, Dong-Hyun Kim, Dong-Min; Jin-O Kim, "Determination of the optimal incentives and amount of load reduction for a retailer to maximize profits considering Demand Response programs", Proceedings of the IEEE Power \& Energy Conference, Dec 2008, pp: 1290-1295.

32. Dam, Q.B., Mohagheghi, S., Stoupis, J., "Intelligent Demand Response Scheme for Customer Side Load Management", Proceedings of the IEEE Energy 2030 Conference, Nov 2008.

33. Mohagheghi, S., Stoupis, J., Wang, Z., Li, Z., Kazemzadeh, H., "Demand Response Architecture: Integration into the Distribution Management System", Proceedings of the IEEE Smart Grid Communications, Oct 2010, pp: 501-506.

34. Markushevich, N., Chan, E., "Integrated Voltage, Var Control and Demand Response in Distribution Systems", Presented at the IEEE Power \& Energy Society, Power Systems Conference and Exposition, Mar 2009.

35. Markushevich N., Berman, A., "Distribution Automation and Demand Response", Presented at the DistribuTech2008 Conference, Jan 2008.

36. Hatziargyriou, N., Asano, H., Iravani, R., Marnay, C., "Microgrids", IEEE Power and Energy Magazine, vol. 5, no. 4, July/Aug 2007, pp: 78-94.

37. Lasseter, R., Abbas, A., Marnay, C., Stevens, J., Dagle, J., Guttromson, R., Sakis Meliopoulos, A., Yinger, R., Eto, J., "Integration of distributed energy resources: The CERTS microgrid concept", California Energy Commission, Oct 2003.

38. Fujioka, Y., Maejima, H., Nakamura, S., Kojima, Y., Okudera, M., Uesaka, S., "Regional power grid with renewable energy resources: A demonstrative project in Hachinohe," CIGRE, August 2006.

39. Basu, A.K., Panigrahi, T.K., Chowdhury, S., Chowdhury, S.P., Chakraborty, N., Sinha, A., Song, Y.H., "Key energy management issues of setting market clearing price (MCP) in micro-grid scenario", Proceedings of the IEEE Universities Power Engineering Conference, Sept 2007, pp: 854-860. 
40. Sinha, A., Basu, A.K., Lahiri, R.N., Chowdhury, S., Chowdhury, S.P., Crossley, P.A., "Setting of market clearing price (MCP) in microgrid power scenario", Proceedings of the IEEE Power \& Energy Society General Meeting, July 2008.

41. Sinha, A., Lahiri, R., Byabortta, S., Chowdhury, S., Crussley, P., "Formulation of pricing mechanism for Microgrid energy", Proceedings of the IET-CIRED Seminar on Smart Grids for Distribution, June 2008.

42. Hatziargyriou, N.D., Dimeas, A., Tsikalakis, A.G., Lopes, J.A.P., Karniotakis, G., Oyarzabal, J., "Management of microgrids in market environment", Proceedings of the International Conference on Future Power Systems, Nov 2005.

43. Sullivan, R.L., "Simulating Central Battery Storage Using a Levelized Incremental Cost Method", IEEE Transactions on Power Apparatus Systems, vol. PAS-101, no. 9, Sept 1982, pp: $3322-3327$.

44. Yau, T., Walker, L.N., Graham, H.L., Gupta, A., Raithel, R., "Effects of Battery Storage Devices on Power System Dispatch", IEEE Transactions on Power Apparatus and Systems, vol. PAS-100, no. 1, Jan 1981, pp: 375-383.

45. Kottick, D., Blau, M., Edelstein, D., "Battery energy storage for frequency regulation in an island power system", IEEE Transactions on Energy Conversion, vol. 8, no. 3, Sept 1993, pp: 455-459.

46. Lo, C.H., Anderson, M.D., "Economic dispatch and optimal sizing of battery energy storage systems in utility load-leveling operations", IEEE Transactions on Energy Conversion, vol. 14, no. 3, Sep 1999, pp: 824829.

47. Mohamed, Faisal A., Koivo, Heikki N., "Online Management of MicroGrid with Battery Storage Using Multiobjective Optimization", Proceedings of the International Conference on Power Engineering, Energy and Electrical Drives, April 2007, pp: 231-236.

48. Zhou, H., Bhattacharya, T., Tran, D., Siew, T.S.T., Khambadkone, A.M., "Composite Energy Storage System Involving Battery and Ultracapacitor with Dynamic Energy Management in Microgrid Applications”, IEEE Transactions on Power Electronics, vol. 26, no. 3, March 2011, pp: 923-930.

49. Guoju, Z., Xisheng, T., Zhiping, Q., "Research on Battery Supercapacitor Hybrid Storage and its application in MicroGrid", Proceedings of the Asia-Pacific Power \& Energy Engineering Conference, March 2010.

50. Erickson, M.J., Lasseter, R.H., "Integration of battery energy storage element in a CERTS microgrid", Proceedings of the IEEE Energy Conversion Congress and Exposition, Sept 2010, pp: 2570-2577. 
51. Maity, I., Rao, S., "Simulation and Pricing Mechanism Analysis of a Solar-Powered Electrical Microgrid”, IEEE Systems Journal, vol. 4, no. 3, Sept 2010, pp: 275-284.

52. Maly, D.K., Kwan, K.S., "Optimal battery energy storage system (BESS) charge scheduling with dynamic programming”, Proceedings of the IEEE Science, Measurement and Technology, vol. 142, no. 6, Nov 1995, pp: 453-458.

53. Tsung-Ying Lee, "Operating Schedule of Battery Energy Storage System in a Time-of-Use Rate Industrial User with Wind Turbine Generators: A Multipass Iteration Particle Swarm Optimization Approach", IEEE Transactions on Energy Conversion, vol. 22, no. 3, Sept 2007 pp: 774-782.

54. Hu, W., Chen, Z., Bak-Jensen, B., “Optimal operation strategy of battery energy storage system to real-time electricity price in Denmark", Proceedings of the IEEE Power \& Energy Society General Meeting, July 2010.

55. Power Smart Pricing 2009 Annual Report - April 27, 2010, Prepared by Summit Blue Consulting, Submitted to Ameren Illinois Utilities.

56. OpenDSS Manual, Electric Power Research Institute, Jul 2010. Available: http://sourceforge.net/projects/electricdss/

57. http://www.smartgrid.epri.com/doc/OpenDSS Level 1 Training.pdf

58. The MathWorks ${ }^{\mathrm{TM}}$, http://www.mathworks.com/products/matlab/description1.html

59. http://www-01.ibm.com/software/integration/optimization/cplex-optimizer/

60. Radial Distribution Test Feeders, IEEE PES Distribution System Analysis Subcommittee

61. Arritt, R. F., Dugan, R. C., “The IEEE 8500-node test feeder”, Proceedings of the IEEE Power \& Energy Society Transmission and Distribution Conference and Exposition, April 2010. 